\title{
Constrained Efficiency with Adverse Selection and Directed Search
}

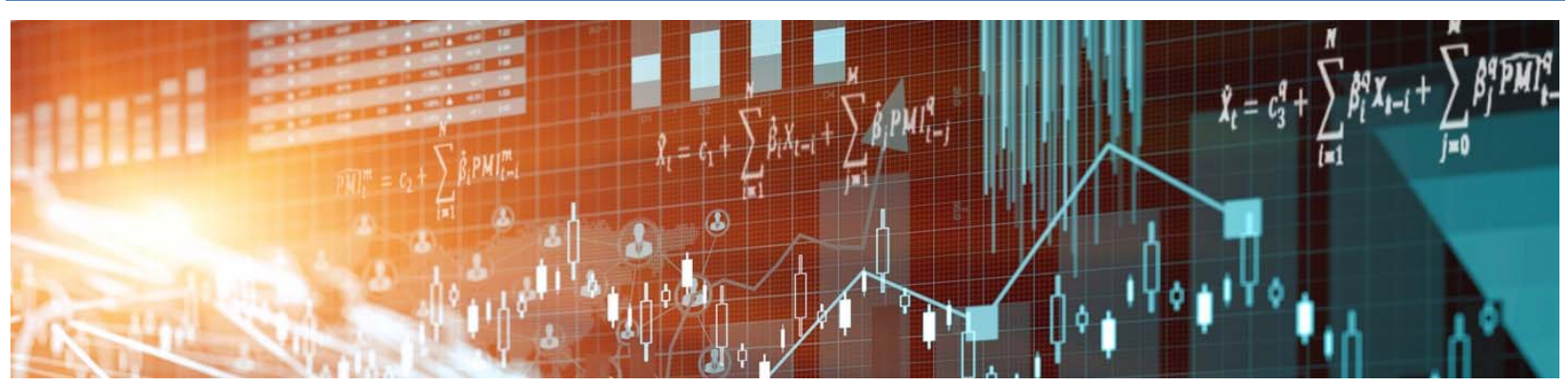

by S. Mohammad R. Davoodalhosseini 
Bank of Canada Staff Working Paper 2017-15

April 2017

\section{Constrained Efficiency with Adverse Selection and Directed Search}

by

S. Mohammad R. Davoodalhosseini

Funds Management and Banking Department

Bank of Canada

Ottawa, Ontario, Canada K1A 0G9

davo@bankofcanada.ca 


\section{Acknowledgements}

This paper was previously circulated under the title "Constrained Efficiency with Search and Information Frictions." I would like to thank Neil Wallace, Manolis Galenianos and Shouyong Shi for their advice, encouragement, and support. I would like also to thank Jonathan Chiu, Kalyan Chatterjee, Ed Green, David Jinkins, Vijay Krishna, Guido Menzio, Francisco Rivadeneyra, Guillaume Rocheteau, Venky Venkateswaran, Russell Wong, Randall Wright, and the participants of the seminars at Penn State University, Cornell-Penn State Macroeconomics Workshop, Southwest Search and Matching Workshop (UCLA), Penn Macro Jamboree, Summer Workshop on Money, Banking, Payments and Finance, and University of Connecticut for their helpful comments and suggestions. 


\begin{abstract}
Constrained efficient allocation (CE) is characterized in a model of adverse selection and directed search (Guerrieri, Shimer, and Wright (2010)). CE is defined to be the allocation that maximizes welfare, the ex-ante utility of all agents, subject to the frictions of the environment. When equilibrium does not achieve the first best (the allocation that maximizes welfare under complete information), then welfare in the CE is strictly higher than welfare in the equilibrium allocation. That is, equilibrium is not constrained efficient. Under some conditions, welfare in the CE even attains welfare in the first best. Finally, sufficient conditions are provided under which equilibrium is not constrained Pareto efficient, either. Cross-subsidization is the key to all these results. In an asset market application, the first best is shown to be implementable through tax schedules that are monotone in the asset prices.
\end{abstract}

Bank topics: Market structure and pricing; Financial system regulation and policies;

Financial markets; Economic models

JEL codes: D82; D83; E24; G1; J31; J64

\title{
Résumé
}

Une allocation efficace opérée sous contrainte est caractérisée dans un modèle de sélection adverse et de prospection ciblée (Guerrieri, Shimer et Wright, 2010). Cette allocation est définie comme une allocation maximisant le bien-être (l'utilité espérée de tous les agents) en présence de frictions. Lorsque l'optimum atteint à l'équilibre n'est pas de premier rang (l'allocation qui maximalise le bien-être en situation d'information parfaite), le bien-être associé à l'allocation efficace est strictement supérieur au bien-être produit par l'allocation d'équilibre. Autrement dit, l'équilibre n'est pas efficace en présence de frictions. À certaines conditions, le bien-être associé à l'allocation efficace est même égal à celui de l'optimum de premier rang. Enfin, nous établissons les conditions sous lesquelles, en présence de frictions, l'équilibre n'est pas un optimum de Pareto. Des subventions croisées expliquent l'ensemble des résultats. Dans le cadre d'un marché d'actifs, nous montrons que l'optimum de premier rang est réalisable si des barèmes de taxes uniformes sont appliqués aux prix des actifs.

Sujets : Structure de marché et fixation des prix; Réglementation et politiques relatives au système financier ; Marchés financiers ; Modèles économiques

Codes JEL : D82 ; D83 ; E24 ; G1 ; J31 ; J64 


\section{Non-Technical Summary}

Adverse selection and search frictions are prevalent in the asset, insurance, labor and housing markets. For example, consider markets for assets traded over the counter such as mortgage-backed securities, structured credit products and corporate bonds. Sellers in these markets may have some private information about the value of their assets, and they must incur search costs to find buyers for their assets.

I study socially efficient allocations in an environment with search frictions and adverse selection using a mechanism design approach: the planner maximizes the weighted average of the payoff to agents subject to the same frictions present in the market economy, namely incentive compatibility and participation constraints of the agents and the planner's budget constraint. The main result is that the market allocation is not constrained efficient. That is, the planner can always achieve higher welfare than the market economy. Also, the planner can sometimes achieve allocations that Pareto dominate the market allocation. Under some conditions, the planner can completely undo the effects of adverse selection and achieve the same welfare level attainable in the economy with complete information. To summarize, an optimal intervention in the form of taxation can improve efficiency of the market.

If the intervention is only in the form of sales tax, then the optimal tax schedule in an asset market application is non-monotone in the asset prices. Implementing a non-monotone tax schedule is difficult from a practical point of view, because it requires the planner to have accurate information about the distribution of types. I show that if entry tax is also used, the optimal allocation can be implemented through sales and entry tax schedules that are monotone in the asset prices.

To understand the mechanism through which the planner can achieve better outcomes, consider a labor market example. There are two types of workers: low type and high type. High-type workers incur less cost for working longer hours and generate higher output (both in average and at the margin). In equilibrium, two types of contracts are offered: a shorthours low-wage contract attracting low-type workers and a long-hours high-wage contract attracting high-type workers. Indeed, high-type workers work inefficiently for longer hours (intensive margin) than they would work under complete information and are matched with inefficiently higher probability, because there is excessive entry of firms (extensive margin). To correct these inefficiencies, the planner uses the policy of taxing the firms that offer long-hours contracts and subsidizing other firms. This policy discourages excessive entry of firms. Also, because of free entry of firms, eventually workers pay or receive these taxes and subsidies. As a result, this policy discourages low-type workers from applying for the longhours contracts. Altogether, this policy corrects the inefficiencies along both the extensive and intensive margins while all constraints, especially the incentive compatibility constraint of low-type workers to apply for the long-hours contract, remain satisfied. 


\section{Introduction}

Adverse selection and search frictions are prevalent in the asset, insurance, labor and housing markets. For example, consider markets for assets traded over-the-counter such as mortgagebacked securities, structured credit products and corporate bonds. Sellers in these markets may have some private information about the value of their assets, and they must incur search costs to find buyers for their assets.

Guerrieri, Shimer, and Wright (2010), GSW hereafter, introduce a model of adverse selection and search frictions. Particularly, they study a directed (competitive) search model in which agents on one side of the market post terms of trade and agents on the other side search for trading partners. Directed search models are interesting and widely used, because the equilibrium allocation with directed search and complete information is usually constrained efficient. Also, directed search may be more realistic than random search in many markets, like the ones mentioned above. Furthermore, as GSW show, using directed search resolves the problem that competitive equilibrium with adverse selection may not even exist (Rothschild and Stiglitz (1976)).

GSW define and characterize equilibrium and show its existence and uniqueness. Because equilibrium in some examples that they present fails to achieve the first best, i.e., the efficient allocation under complete information, a natural and important question is whether it is possible to achieve "better" allocations than the market, with the planner's intervention consistent with the frictions of the environment. To address such questions, I study socially efficient allocations in their environment by using a mechanism design approach. In particular, I study whether the equilibrium allocation is socially efficient or not. And, what is the best allocation that the planner can achieve?

The environment can be briefly described as one with a large number of buyers whose population is endogenously determined through free entry. There is a fixed population of sellers of $I \in \mathbb{N}$ types who have private information about their types. Buyers and sellers match bilaterally and trade in different locations, called submarkets. In each submarket, there are search frictions in the sense that buyers and sellers on both sides are matched generally with probability less than one.

To study constrained efficiency in this environment, consider a planner who chooses an allocation that maximizes welfare, the ex-ante payoff to all types, subject to the frictions of the environment. Call this allocation the constrained efficient allocation (CE). In Theorem 1, I show that the planner achieves strictly higher welfare than equilibrium for any distribution of types when equilibrium does not achieve the first best (ex-ante incentive inefficiency of equilibrium). In Theorem 3, I show that the planner can even achieve the first best under

relatively mild conditions. These results are important as they imply that although adverse selection can lead to inefficiency, appropriate interventions can completely undo the effects of 
adverse selection and recover efficiency. In Theorem 4, I provide necessary conditions for the CE. This result is useful for the environments in which the sufficient conditions of Theorem 3 may not hold. Pinning down the CE from these necessary conditions is usually easy in economic applications. Finally, in Theorem 2, I show that if the measure of lower types is sufficiently small, then the equilibrium allocation is not constrained Pareto efficient (interim incentive inefficiency of equilibrium). That is, there exists an allocation that satisfies all participation and incentive constraints of the agents and Pareto dominates the equilibrium. This result is reminiscent of a well-known result that competitive equilibrium with adverse selection, even if it exists, may not be constrained Pareto efficient (e.g., Greenwald and Stiglitz (1986), among others).

The key to all these results is cross-subsidization. That is, some types should be taxed to finance subsidies to other types. The important task in the proofs is to show how crosssubsidization should be used, i.e., how the contracts allocated to different types should be changed compared with equilibrium and how much transfers different types should pay or receive. First, I show, similar to GSW, that it is sufficient to consider only some incentive compatibility (IC) constraints, only the upward IC constraints, to characterize equilibrium. The upward (downward) IC constraint means that a lower (higher) type should not gain by reporting a higher (lower) type. Second, I show that the downward IC constraints are not binding in equilibrium. That is, all types strictly prefer to report their own type rather than a lower one. These two points together provide a great deal of flexibility for the planner to construct better allocations: if lower types are equally subsidized by a sufficiently small amount, then none of the IC constraints are violated.

To construct an allocation with higher welfare than the equilibrium allocation, assuming that type $i$ 's equilibrium allocation is different than the first best allocation, the planner subsidizes types $1,2, \ldots, i-1$ by a small amount. To finance these subsidies, the planner taxes all types in a lump sum way. Since the amount of this lump sum tax is small too, the participation constraints of agents are not affected. The IC constraints are not affected either as mentioned above, so this allocation respects all the constraints that the planner faces (budget-balance, participation and incentive constraints). Therefore, welfare in this allocation is higher than welfare in the equilibrium allocation, as type $i$ creates more surplus due to the relaxation of upward IC constraints of lower types, and other types continue to create the same amount of surplus.

To construct an allocation that Pareto dominates the equilibrium allocation, the same schedule of subsidies is used as above, but they are now financed by taxing only types $1,2, \ldots, i$. If the level of improvement in the type $i$ 's payoff resulting from the relaxation of IC constraints is greater than the amount of tax that type $i$ should pay, then this allocation will Pareto dominate the equilibrium allocation. This happens if the aggregate measure of types $1,2, \ldots, i-1$ is sufficiently smaller than the measure of type $i$. 
There has been much discussion post-financial crisis about the role of private information in causing this crisis. Consequently, many policy questions have arisen, one of which is whether subsidizing asset purchases is a good policy from a social point of view. To the best of my knowledge, no paper has studied this question yet, but some papers such as Chang (2014), Guerrieri and Shimer (2014a) and Chiu and Koeppl (2016) have studied positive implications of these and similar policies. Guerrieri and Shimer (2014a) study the effects of asset subsidy programs by an entity with deep pockets and show that these programs can increase liquidity and prices of assets, therefore saving the market from a liquidity crisis. Similarly, Chang (2014) shows that the purchase of low-price assets increases the liquidity of all assets in the market. None of the above papers consider budget-balanced policies, nor do they study the optimal one. ${ }^{1}$

To characterize the optimal policy that is budget-balanced in these markets, I study a model of asset market with lemons. Sellers have one indivisible asset that is of two types. The high-type asset is more valuable to both buyers and sellers. In the unique equilibrium, which is separating, sellers with high-type assets strictly prefer to trade in the submarket with a higher price but lower probability of finding a buyer. Sellers with low-type assets, conversely, sell their assets in the submarket with a low price but higher probability of finding a buyer. These sellers, indeed, are indifferent between the two submarkets.

The optimal policy is to subsidize trade in the low-price submarket and tax trade in the high-price one so that low-type sellers strictly prefer the former to the latter. Since the IC constraint of low-type sellers to go to the high-price submarket is now relaxed, more buyers are willing to enter the high-price one, increasing the chances of high-type sellers to meet a buyer. As a result, welfare increases as the volume of trade increases compared with the equilibrium allocation. Furthermore, if the fraction of low-type sellers in the population is sufficiently small, then the same cross-subsidization scheme can be used to construct an allocation that Pareto dominates the equilibrium.

This two-type model is extended to a continuous-type one to investigate whether the optimal submarket-specific sales tax is monotone in the asset prices, similar to the two-type model. It turns out that this is not the case. In the next step, I show that imposing two types of taxes, sales tax and submarket-specific entry tax, solves the non-monotonicity problem. Entry tax is levied on buyers conditional on entry to each submarket whether or not they find a match. This result states that the planner can always design monotone tax schedules, decreasing entry tax and increasing sales tax, to implement the CE.

This paper is related to the literature on directed as well as random search. It is well

\footnotetext{
${ }^{1}$ Since the budget-balance condition is taken into account in the present paper, its analysis is specially useful for long-run interventions, when budget considerations are important. The policy analyses in the aforementioned papers seem to be more concerned with short-time (crisis management) policies, so they do not restrict policies to be budget balanced.
} 
established in the literature that the agents entering the market in the directed search settings can internalize search externalities by choosing the "right" price (contract) and committing to them, if the agents' types are observable and contractible. The following papers study directed search: Peters (1991), Moen (1997), Acemoglu and Shimer (1999a), Shi (2001, 2002), Mortensen and Wright (2002), Shimer (2005) and Eeckhout and Kircher (2010), among many others. In the random search settings, the equilibrium is generally inefficient because the entrants generally fail to internalize the search externalities. See Pissarides (2000) for a random search model.

My paper is the first in the directed search literature that uses a mechanism design approach. The only paper that is somewhat an exception is Golosov, Maziero, and Menzio (2013), who study a model with directed search and moral hazard. The information asymmetry in their paper is not about gains from trade; rather, it is about workers' search decision. More specifically, workers have private information whether they have searched or not, and if they have searched, toward which type of firms. Guerrieri (2008) and Moen and Rosén (2011) study the constrained efficient allocation in environments with directed search and private information. In both papers the agents who search (workers) do not have ex-ante private information. Rather, their information is match-specific and realized only after they are matched with firms.

The idea that cross-subsidization helps to relax incentive constraints has been used in the adverse selection literature (e.g., Miyazaki (1977) and Spence (1978)). I elaborate on the relationship between my results and important contributions in this literature, namely, Rothschild and Stiglitz (1976), Wilson (1977), Holmström and Myerson (1983) and Maskin and Tirole (1992), in Section 6, but my contributions to this literature can be summarized as follows: First and foremost, I show that the inefficiency of equilibrium in standard adverse selection models fully extends to environments with search and matching. ${ }^{2}$ Second, my results regarding ex-ante and interim inefficiency of equilibrium are more general compared with other results in this literature, if one incorporates the matching probability into the payoff function of agents and treats it as another dimension of contract space. My results are more general as I do not impose any restrictions on the number of types or dimension of contract space (except being finite) or concavity of payoff functions. Third, although the idea that cross-subsidization is helpful is not new, my results shed light on the way that cross-subsidization should be used.

The paper is organized as follows. In Section 2, I develop the environment of the model and define the planner's problem. In Section 3, I state my main results. In Section 4, I study a two-type asset market application, characterize the constrained efficient allocation and compare it with the equilibrium allocation. I also explain the nature of inefficiency in the

\footnotetext{
${ }^{2}$ Note that traditional search frictions are not necessary for my results, so even if the short side of the market matches for sure, the results continue to hold.
} 
market economy and discuss why and how the planner can allocate resources more efficiently than the market economy. In Section 5, I study a version of the rat race, originally studied by Akerlof (1976). The planner achieves the first best by paying low-type workers higher wages and high-type workers lower wages than their wages under complete information. If the fraction of high-type workers is sufficiently high, then CE even Pareto dominates the equilibrium allocation. In Section 6, I place my results in the adverse selection literature. In Section 7, I study the asset market with a continuous-type space and discuss the monotonicity of the optimal tax schedule. Section 8 concludes. All proofs appear in the Appendix unless otherwise noted.

\section{$2 \quad$ Model}

I introduce the environment of the model first and then define the planner's problem. My environment is similar to that of GSW with two minor differences: I impose quasi-linearity on the payoff functions, mostly to make the exposition simpler, and also I do not allow for lotteries on allocations. ${ }^{3}$ In the Appendix, I elaborate on why I impose these assumptions, but it is enough to say here that all results will continue to hold even without them.

\section{$2.1 \quad$ Environment}

Consider an economy with two types of agents - buyers and sellers - and $n+1$ goods where $n \in \mathbb{N}$. Goods $1,2, \ldots, n$ are produced by sellers and consumed by buyers, while good $n+1$ is a numeraire good and is produced and consumed by everyone. Let $a \equiv\left(a^{1}, a^{2}, \ldots, a^{n}\right) \in \mathbb{A} \subset \mathbb{R}^{n}$ be a vector where $\mathbb{A}$ is compact, convex and non-empty. Component $l$ of this vector, $a^{l}$, denotes the quantity of good $l$. For example, in a labor market, $a$ can be a positive real number denoting the hours of work. When I say an agent produces (or consumes) $a$, I mean that the agent produces (or consumes) $a^{1}$ units of good $1, a^{2}$ units of good 2 and so on.

There is a measure 1 of sellers. A fraction $\pi_{i}>0$ of sellers are of type $i \in\{1,2, \ldots, I\}$. Type is seller's private information. On the other side of the market, there is a large continuum of homogenous buyers who can enter the market by incurring cost $k>0$. After buyers enter the market, buyers and sellers are allocated to different submarkets (described below). Matching is bilateral. After they match, they trade.

There are search frictions in this environment. By search frictions, I mean that sellers generally get to match with the buyers they have chosen with probability less than one.

\footnotetext{
${ }^{3}$ By lotteries, I mean that the same type of sellers are assigned to different allocations with positive probabilities when these allocations deliver them different payoffs. However, I do allow for lotteries to be used within a given allocation. For example, an allocation may specify that a seller of a given type gets paid a certain amount of money (numeraire good) but delivers the asset with a probability less than one. My assumption only requires that this allocation be the same for all sellers of this type.
} 
Matching occurs in submarkets that are simply some locations for trade. Matching technology determines the probability that sellers and buyers in each submarket are matched. If the ratio of buyers to sellers in one submarket is $\theta \in[0, \infty]$, then the buyers are matched with probability $q(\theta)$. Symmetrically, matching probability for sellers is $m(\theta) \equiv \theta q(\theta)$. As standard in the literature, I assume that $m$ is non-decreasing and $q$ is non-increasing. Both $m$ and $q$ are continuous.

Sellers' and buyers' payoff functions are quasi-linear in the numeraire good. The payoff to a buyer who enters the market from consuming $a$ and producing $p \in \mathbb{R}$ units of the numeraire good is $v_{i}(a)-p-k$ if matched with a type $i$ seller, and is $-k$ if unmatched. The payoff to a type $i$ seller from producing $a$ and consuming $p \in \mathbb{R}$ units of the numeraire good is $u_{i}(a)+p$ if matched with a buyer and is 0 otherwise.

\subsection{Planner's Problem Using Taxation}

I first briefly describe how the market economy works, the special case in which the planner does nothing, and then define the planner's problem. Submarkets in the market economy are characterized by $y \equiv(a, p)$, where $a \in \mathbb{A}$ denotes the vector of goods 1 to $n$ to be produced by sellers in this submarket and $p \in \mathbb{R}$ is the amount of the numeraire good to be transferred from buyers to sellers. No submarket that would deliver buyers a strictly positive payoff is inactive in the equilibrium. On the other side of the market, sellers observe all $(a, p)$ pairs posted in the market, anticipate the market tightness for each submarket and then direct their search toward one that delivers them the highest expected payoff. The equilibrium notion used here is exactly the same as that in GSW. ${ }^{4}$

The planner is assumed to have the power to levy taxes and subsidies on agents. First, the planner sets a tax amount for each submarket. This tax will be levied on buyers conditional on trade, $t(a, p): \mathbb{A} \times \mathbb{R} \rightarrow \mathbb{R}$. The results will not change if, instead, taxes are levied on sellers. Second, the planner makes lump sum transfers, $t_{0} \in \mathbb{R}_{+}$units of the numeraire good, to sellers. Call $\left\{t, t_{0}\right\}$ a policy. In the next subsection, the planner's problem is defined using a direct mechanism in which sellers report their types to the planner and then the planner allocates them resources. I show that levying these two types of taxes is sufficient for the planner to implement the outcome of the direct mechanism.

Let $\gamma_{i}(y)$ denote the share of sellers who are type $i$ in the submarket denoted by $y$, with $\Gamma(y) \equiv\left\{\gamma_{1}(y), \ldots, \gamma_{i}(y), \ldots, \gamma_{I}(y)\right\} \in \Delta^{I}$ where $\Delta^{I}$ is an $I$-dimensional simplex, that

\footnotetext{
${ }^{4}$ When I refer to equilibrium in the paper, I mean the notion of equilibrium in which the uninformed side of the market posts contracts as discussed above. I do not mean the notion of equilibrium with signaling in which the informed side of the market posts contracts, as in Delacroix and Shi (2013). I conjecture that my results regarding the inefficiency of equilibrium will hold even if the latter notion is considered. However, one may need to impose some reasonable restrictions on off-the-equilibrium-path beliefs similar to those proposed by Cho and Kreps (1987).
} 
is, $0 \leq \gamma_{i}(y) \leq 1$ for all $y$ and $\sum_{i=1}^{I} \gamma_{i}(y)=1$. To make the notation clear for the rest of the paper, the first component of $y \equiv(a, p)$ is denoted by $a$ rather than $y_{1}$, and the second component is denoted by $p$ rather than $y_{2}$. Similarly, if a submarket is denoted by $y^{\prime}$, the first and second components of $y^{\prime}$ are denoted by $a^{\prime}$ and $p^{\prime}$. An allocation $\{\lambda, \mathcal{Y}, \Theta, \Gamma\}$ is a distribution $\lambda$ over $Y$ with support $\mathcal{Y}$ (so $\mathcal{Y}$ is the set of open or active submarkets), the ratio of buyers to sellers for each submarket $\Theta: Y \rightarrow[0, \infty]$, and the distribution of types in each submarket $\Gamma: Y \rightarrow \Delta^{I}$. Because the planner faces some constraints, only some allocations are implementable, which are defined below.

Definition 1. An allocation $\{\lambda, \mathcal{Y}, \Theta, \Gamma\}$ is implementable through policy $\left\{t, t_{0}\right\}$ if it satisfies conditions (i)-(iv):

(i) Buyers' maximization and free entry: For any $y \equiv(a, p) \in Y$,

$$
q(\Theta(y)) \sum_{i} \gamma_{i}(y)\left(v_{i}(a)-p-t(y)\right) \leq k, \text { with equality if } y \in \mathcal{Y}
$$

(ii) Sellers' optimal search: Let $U_{i} \equiv \max \left\{0, \max _{y^{\prime} \in \mathcal{Y}}\left\{m\left(\Theta\left(y^{\prime}\right)\right)\left(u_{i}\left(a^{\prime}\right)+p^{\prime}\right)\right\}\right\}+t_{0}$ and $U_{i}=t_{0}$ if $\mathcal{Y}=\emptyset$. For any $y \in Y$ and $i$,

$$
m(\Theta(y))\left(u_{i}(a)+p\right)+t_{0} \leq U_{i} \text {, with equality if } \gamma_{i}(y)>0 \text { and } \Theta(y)<\infty .
$$

If $u_{i}(a)+p<0$, then $\Theta(y)=\infty$ or $\gamma_{i}(y)=0$.

(iii) Feasibility or market clearing: For all $i, \int_{\mathcal{Y}} \frac{\gamma_{i}(y)}{\Theta(y)} d \lambda(\{y\}) \leq \pi_{i}$, with equality if $U_{i}>t_{0}$.

(iv) Planner's budget-balance condition: $\int_{\mathcal{Y}} q(\Theta(y)) t(y) d \lambda(\{y\}) \geq t_{0}$.

An allocation is said to be implementable if it is implementable through some policy.

Some comments about this definition will follow. Note that under the policy of zero taxes, i.e., $t(y)=0$ for all $y \in Y$ and $t_{0}=0$, this definition reduces exactly to the definition of equilibrium in GSW, so the equilibrium in GSW is clearly implementable through the policy of zero taxes. In general, buyers take into account the policy, i.e., the tax amount for each submarket, and then decide where to go. Sellers then choose which submarket to go to among all submarkets that buyers have chosen. Condition (i) states that buyers should not earn a strictly positive profit from entering any submarket (on- or off-the-equilibrium-path). Moreover, for all markets that the planner wants to be open, buyers must receive exactly zero expected payoff. A buyer has to incur entry cost $k$ if he wants to enter submarket $y$. Then, he matches with a type $i$ seller with probability $\gamma_{i}(y)$ from which he receives a payoff of $v_{i}(a)$ in terms of the numeraire good, and pays $p$ units of the numeraire good to the seller and $t(y)$ units to the planner.

Condition (ii) is composed of two parts. The first part states that among all open submarkets, $y \in \mathcal{Y}$, sellers choose to go to a submarket that maximizes their payoff. The second 
part imposes some restrictions on beliefs regarding the market tightness and composition of types for off-the-equilibrium-path, $y \notin \mathcal{Y}$. The market tightness for off-the-equilibrium-path is set such that the sellers who choose to go to those submarkets do not gain by doing so relative to their equilibrium payoff. Also, this restriction with respect to the composition of types states that if buyers believe that some types would apply to an off-the-equilibrium-path submarket, then those types should be exactly indifferent between the payoff they receive from that submarket relative to their equilibrium payoff. Condition (iii) is straightforward. Condition (iv) is the planner's budget-balance condition stating that no external resources are available to the planner to finance the transfers.

In the market economy, it is not possible to transfer funds (the numeraire good) from one submarket to another. In contrast, the planner here may want to levy taxes on agents in some submarkets and subsidize others, so sellers might receive a higher or lower payoff than the surplus they generate. This cross-subsidization across submarkets is the key to all the results. The difficulty in the proofs comes from the fact that any cross-subsidization scheme affects incentive constraints of all types, so it should be conducted in a way so that all constraints remain satisfied.

Definition 2. A constrained efficient allocation is an implementable allocation that maximizes welfare among all implementable allocations. That is, a constrained efficient allocation solves the following problem:

$$
\max _{\{\lambda, \mathcal{Y}, \theta, \Gamma\},\left\{t, t_{0}\right\}} \sum_{i} \pi_{i} U_{i}
$$

subject to $\{\lambda, \mathcal{Y}, \theta, \Gamma\}$ is implementable through policy $\left\{t, t_{0}\right\}$,

where $U_{i}$, type i's payoff, is defined in part (ii) of Definition 1.

In the language of mechanism design, the notion of efficiency used above is ex-ante incentive efficiency, i.e., welfare is calculated before agents learn their types. Another possible notion is interim incentive efficiency in which welfare is calculated after each agent learns his type. An allocation is interim incentive efficient if it is implementable and there does not exist another implementable allocation in which all types are weakly better off while some types are strictly better off, i.e., if no other implementable allocation Pareto dominates it. Since in my model agents know their types from the beginning, one may argue that the appropriate notion is an interim one. I would argue that although interim efficiency is an appropriate notion here, ex-ante efficiency is appropriate too.

One way to think about ex-ante efficiency is to assume that the sellers in my model meet before learning their types and try to design the best arrangements for trade. These sellers maximize their ex-ante payoff taking into account that after the realization of their types, they will have private information. Another way to think about ex-ante efficiency is to assume that sellers are members of a family or employees of a large firm who compete 
with one another in the market stage, but share their payoffs after they trade in the market. Either way, they want to maximize the aggregate amount of the surplus created in this economy given the incentive constraints. I elaborate on these notions of efficiency in Section 6.

In Theorems 1, 3 and 4 below, ex-ante efficiency is concerned. ${ }^{5}$ However, in Theorem 2 (and all the applications), sufficient conditions will be provided under which the equilibrium allocation is not only ex-ante incentive inefficient but also interim incentive inefficient. ${ }^{6}$

\subsection{Planner's Problem Using Direct Mechanism}

In this section, the planner is assumed to use a direct mechanism to allocate resources. In the direct mechanism, sellers report their types to the planner and then the planner allocates them to a 3-touple $\left(\theta_{i}, a_{i}, s_{i}\right)$. Intuitively, the planner sets up $I$ locations (one for each type), a certain number of buyers are allocated to match with sellers at each location and then sellers are asked to produce a certain amount of goods if they find a match. Also, the planner makes transfers to sellers.

More precisely, $a_{i}$ is the vector of goods 1 to $n$ to be produced by sellers who report type $i$ conditional on finding a match in the location designed for type $i, s_{i}$ is the amount of the numeraire good transferred to them and $\theta_{i}$ is the average number of buyers assigned to them in that location. The planner is subject to the same frictions present in the market economy, as summarized below:

Definition 3. A feasible mechanism is a set $\left\{\left(\theta_{i}, a_{i}, s_{i}\right)\right\}_{i \in\{1,2, \ldots, I\}}$ such that the following conditions hold:

(i) Incentive compatibility of sellers: for all $i$ and $j$

$$
U_{i} \equiv m\left(\theta_{i}\right) u_{i}\left(a_{i}\right)+s_{i} \geq m\left(\theta_{j}\right) u_{i}\left(a_{j}\right)+s_{j}(I C) .
$$

(ii) Participation constraint of sellers: for all $i$

$$
U_{i} \geq 0(P C) \text {. }
$$

(iii) Planner's budget-balance condition:

$$
\sum_{i=1}^{I} \pi_{i}\left[m\left(\theta_{i}\right) v_{i}\left(a_{i}\right)-k \theta_{i}\right] \geq \sum_{i=1}^{I} \pi_{i} s_{i}(B B) .
$$

\footnotetext{
${ }^{5}$ When the planner or constrained efficiency is mentioned without a further reference, the ex-ante notion of efficiency is meant.

${ }^{6}$ Focusing on the ex-ante efficiency notions is consistent with long-run efficiency considerations in many environments, such as some financial markets, in which sellers may have superior information compared with buyers at any point in time, but in the long run, participants may want to collectively set up arrangements to improve efficiency. On the other hand, focusing on the notion of interim efficiency discussed in Theorem 2 may be more appropriate for environments such as housing or labor markets in which the type of agents does not change frequently.
} 
These conditions are straightforward to understand. The only point is that in condition (iii), the right-hand-side (RHS) is the amount of the numeraire good that the planner must pay to sellers. The left-hand-side (LHS) is the net payoff to buyers from entry added over all $I$ locations, as there $\pi_{i} \theta_{i}$ number of buyers at each location, and each of them gets an expected benefit of $q\left(\theta_{i}\right) v_{i}\left(a_{i}\right)-k$. The planner's objective is to maximize welfare, which is the weighted average of the payoff to sellers. ${ }^{7}$

Definition 4. An optimal mechanism, $\left\{\left(\theta_{i}^{*}, a_{i}^{*}, s_{i}^{*}\right)\right\}_{i \in\{1,2, \ldots, I\}}$, is a feasible mechanism that maximizes welfare. That is, the optimal mechanism solves the following problem:

$$
\max _{\left\{\left(\theta_{i}, a_{i}, s_{i}\right)\right\}_{i \in\{1,2, \ldots, I\}}} \sum_{i} \pi_{i}\left(m\left(\theta_{i}\right) u_{i}\left(a_{i}\right)+s_{i}\right)
$$

subject to: $\left\{\left(\theta_{i}, a_{i}, s_{i}\right)\right\}_{i \in\{1,2, \ldots, I\}}$ is a feasible mechanism.

Using a direct mechanism requires a large amount of communication and may not be practical in many economic applications, but working with it is convenient. Lemma 1 guarantees that there is no loss of generality in focusing only on direct mechanisms. That is, a planner who uses a direct mechanism achieves the same welfare level as the planner in Definition 2. Therefore, all results obtained by utilizing direct mechanisms can be naturally obtained in the market setting if the planner has the power of taxation.

Lemma 1. For any feasible mechanism, there is an associated implementable allocation under which all types receive exactly the same payoff as in the direct mechanism.

The intuition behind this result is simple. Since there is no constraint on the taxation power of the planner, the planner can effectively shut down any submarket of his choice by levying large taxes on that submarket to achieve the same outcome as in the direct mechanism. If some restrictions are imposed on the taxation power, e.g., a ceiling on the amount of taxes at each submarket or the monotonicity of taxes as a function of price, then this result may not hold. Moreover, given that the taxation power is not restricted here, constrained efficient allocation is invariant to any restriction on the off-the-equilibrium-path beliefs, again, because the planner can prevent trading in any submarket that he wants by levying large taxes on trade in that submarket, and as a result, no buyer would want to trade in that submarket no matter how off-the-equilibrium-path beliefs are formed.

\section{Characterization}

The complete information allocation is first studied as a benchmark and then the main results are presented.

\footnotetext{
${ }^{7}$ Buyers receive zero payoff both in the market economy and under the planner's problem, so it is equivalent to say that the planner's objective is to maximize the total amount of the surplus in the economy.
} 


\subsection{Complete Information Allocation or First Best}

As a benchmark, consider an otherwise identical environment as introduced above except that the type of sellers is common knowledge. The optimal mechanism with complete information can be defined in a similar fashion as in Definition 3. The only difference is that incentive compatibility constraint should be removed from the definition of feasible mechanism. Without IC constraints, the planner with complete information always achieves weakly higher welfare than the planner facing private information. It is also easy to see that the optimal mechanism with complete information can be solved type by type. That is, the planner with complete information solves the following problem for each type separately:

$$
\max _{\theta, a, s}\left\{m(\theta) u_{i}(a)+s\right\}
$$

subject to: $m(\theta) v_{i}(a)-s \geq k \theta$, and $m(\theta) u_{i}(a)+s \geq 0$.

Let $U_{i}^{F B}$ be the optimal value for this problem, so

$$
U_{i}^{F B}=\max _{\theta, a}\left\{m(\theta)\left(v_{i}(a)+u_{i}(a)\right)-k \theta\right\} .
$$

Moreover, define

$$
\left(\theta_{i}^{F B}, a_{i}^{F B}\right) \in \arg \max _{\theta, a}\left\{m(\theta)\left(v_{i}(a)+u_{i}(a)\right)-k \theta\right\} .
$$

It is easy to check that $U_{i}^{F B}$ is exactly the payoff to the sellers of type $i$ in the equilibrium with complete information. ${ }^{8}$ The first best allocation $(F B)$ is defined to be an allocation in which type $i$ sellers produce $a_{i}^{F B}$ and match in a location with market tightness $\theta_{i}^{F B}$.

Regarding the language used in the paper, it is said that the planner achieves the first best if there exists a feasible mechanism $\left\{\left(\theta_{i}, a_{i}, s_{i}\right)\right\}_{i \in\{1,2, \ldots, I\}}$ in which $\left(\theta_{i}, a_{i}\right) \in \arg \max _{\theta, a}\left\{m(\theta)\left(v_{i}(a)+\right.\right.$ $\left.\left.u_{i}(a)\right)-k \theta\right\}$ for all $i$. Equivalently, it is said that the planner achieves the first best if the first best allocation is implementable. Moreover, it is said that the equilibrium achieves the first best (does not achieve the first best) if FB is (not) implementable through the policy of zero taxes, i.e., $t()=$.0 and $t_{0}=0$. Moreover, when equilibrium is used without any

\footnotetext{
${ }^{8}$ In the equilibrium with complete information, buyers post $(a, p, i)$ to attract type $i$ sellers. Therefore, they solve $\max _{\theta, a, p}\left\{m(\theta)\left(u_{i}(a)+p\right)\right\}$ subject to $q(\theta)\left(v_{i}(a)-p\right) \geq k$. This problem is clearly the same as the planner's problem with complete information. As cited in the introduction, there are many papers in the literature with different environments but with the common theme that when agents on one side of the market compete with each other in posting contracts and commit to them, then the market decentralizes the planner's allocation, if the contract space is rich enough. If the contract space is not rich enough, the equilibrium might be constrained inefficient, as in Galenianos and Kircher (2009). Moreover, Acemoglu and Shimer (1999b) and, more specifically, Golosov et al. (2013) find inefficiency of directed search equilibrium under some types of moral hazard in the context of labor markets. Following the present paper, Davoodalhosseini (2015) uses a directed search model with adverse selection and complementarity between factors of production and shows that equilibrium is constrained inefficient.
} 
reference, GSW equilibrium is meant. As far as the notation is concerned, superscript * represents the optimal mechanism (or constrained efficient allocation), FB represents the first best allocation and EQ represents the GSW equilibrium allocation.

\subsection{Efficiency Properties of GSW Equilibrium}

Since GSW equilibrium is an implementable allocation through the policy of zero taxes, it is immediately followed that the planner can achieve the welfare level that is at least as much as that in the equilibrium. Theorem 1 states that the planner can achieve strictly higher welfare than the equilibrium regardless of the distribution of types. The obvious qualification is that the equilibrium should fail to achieve the first best, for otherwise, private information would not have caused any inefficiency and the planner would not be able to achieve higher welfare. $^{9}$

Let $\bar{Y} \equiv \cup_{i} \bar{Y}_{i}$ where $\bar{Y}_{i} \equiv\left\{(a, p) \mid(a, p) \in \mathbb{A} \times \mathbb{R}, q(0)\left(v_{i}(a)-p\right) \geq k\right.$, and $\left.u_{i}(a)+p \geq 0\right\}$, and $\bar{A} \equiv\{a \mid(a, p) \in \bar{Y}$ for some $p \in \mathbb{R}\}$. If $(a, p) \notin \bar{Y}$, then no type will be attracted to this submarket in the market economy.

\section{Assumption 1.}

(i) Strict monotonicity: For all $a \in \bar{A}, v_{1}(a)<v_{2}(a)<\ldots<v_{I}(a)$.

(ii) Sorting: For all $i, a \in \bar{A}$ and $\epsilon>0$, there exists $a^{\prime} \in B_{\epsilon}(a) \equiv\left\{a^{\prime} \in \mathbb{A} \mid\left\|a-a^{\prime}\right\|_{2}<\epsilon\right\}$ such that

$$
u_{j}\left(a^{\prime}\right)-u_{j}(a)<u_{h}\left(a^{\prime}\right)-u_{h}(a) \text { for all } j \text { and } h \text { with } j<i \leq h .
$$

(iii) Single peak: $m(\theta)\left(u_{i}(a)+v_{i}(a)\right)-k \theta$ has a single peak on its domain, $\mathbb{R}_{+} \times \mathbb{A}$, for all $i$.

Theorem 1 (Ex-ante incentive inefficiency of equilibrium). Suppose Assumption 1 holds. Also assume that all types with positive gains from trade, i.e., all $i$ with $U_{i}^{F B}>0$, receive a strictly positive payoff in equilibrium. If equilibrium fails to achieve the first best, then welfare in the constrained efficient allocation is strictly higher than that in equilibrium.

Some remarks about the assumptions are in order. Assumption 1(ii) is the same as Assumption A3 in GSW. A sufficient condition for Assumption 1(iii) is that $m(\theta)\left(u_{i}(a)+\right.$ $\left.v_{i}(a)\right)-k \theta$ is strictly quasi-concave. Furthermore, if one assumes that $m$ is concave, as usually assumed in the applications, then a milder sufficient condition for Assumption 1(iii) is that $u_{i}(a)+v_{i}(a)$ is strictly quasi-concave. The requirement that all types with positive gains from trade must be active in equilibrium is satisfied if there are positive gains from

\footnotetext{
${ }^{9}$ Assuming that $U_{i}^{F B}>0$ for all $i$, equilibrium fails to achieve the first best if and only if $U_{i}^{F B}-U_{j}^{F B}>$ $m\left(\theta_{i}^{F B}\right)\left(u_{i}\left(a_{i}^{F B}\right)-u_{j}\left(a_{i}^{F B}\right)\right)$ for some $i, j$. As a result, one needs to check at most $n(n-1)$ conditions to examine whether equilibrium fails to achieve the first best or not.
} 
trade for all types (according to Proposition 4 in GSW). In an example in Section 4, I make it clear why this assumption is necessary.

The idea of the proof will follow. I begin from the equilibrium allocation, propose a direct mechanism, which basically implements an allocation sufficiently close to the equilibrium allocation, and then show that the proposed allocation is feasible and achieves strictly higher welfare than the equilibrium allocation.

First, we need to understand how GSW equilibrium is constructed. Let $\left\{\lambda^{E Q}, \mathcal{Y}^{E Q}, \Theta^{E Q}, \Gamma^{E Q}\right\}$ denote the equilibrium allocation where $\mathcal{Y}^{E Q} \equiv\left\{y_{1}^{E Q}, y_{2}^{E Q}, \ldots, y_{I}^{E Q}\right\}$. Also let $U_{i}^{E Q}$ denote the payoff to type $i$ in the equilibrium. In this explanation, assume that all types are active in the equilibrium, $U_{i}^{E Q}>0$. Under similar conditions, weak monotonicity and sorting, GSW prove that the equilibrium for type $i$ is uniquely characterized by maximizing the payoff to type $i$ subject to the free-entry condition and the incentive compatibility constraint of all lower types. After incorporating the free-entry condition, the problem turns into:

$$
\max _{\theta \in[0, \infty],(a, .) \in \bar{Y}}\left\{m(\theta)\left(u_{i}(a)+v_{i}(a)\right)-k \theta\right\}
$$

subject to: $m(\theta)\left(u_{j}(a)+v_{i}(a)\right)-k \theta \leq U_{j}^{E Q}\left(\mathrm{IC}_{j i}\right)$ for all $j<i$.

Call this problem $P_{i}$. The objective function is the surplus that type $i$ creates. The constraint $I C_{j i}$ states that type $j$ should not receive a higher payoff if he chooses the submarket that type $i$ chooses, for all $j<i$. See the proof of Theorem 1 for derivation of this problem.

Since the equilibrium does not achieve the first best, there must exist a type, say type $r$, that creates a surplus that is strictly less than the first best level, i.e., $U_{r}^{E Q}<U_{r}^{F B}$. It implies that at least one incentive compatibility constraint in the problem for type $r$ is binding in the equilibrium.

The planner begins from a direct mechanism in which each type is allocated the same production level $a$, the same market tightness $\theta$ and the same expected payment $s=m(\theta) p$ (where $p=v_{i}(a)-\frac{k}{q(\theta)}$ is pinned down by free-entry condition) as in equilibrium. The planner then subsidizes all types below $r$ identically by a small amount, $\epsilon>0$. Since the constraints of the maximization problem for type $r$ become slack, the planner can find another triple $\left(\theta^{\prime}, a^{\prime}, s^{\prime}\right)$ close to $(\theta, a, s)$ for this type such that the surplus generated by this type increases following Assumption 1(iii). ${ }^{10}$ As a result, the payoff to type $r$ strictly increases.

To ensure that these transfers do not violate IC constraints, it is shown in Lemma 3 in the Appendix that no downward IC constraint in the equilibrium is binding; that is, type

\footnotetext{
${ }^{10}$ It can be clearly seen here why Assumption 1(iii) is needed. If $m(\theta)\left(u_{i}(a)+v_{i}(a)\right)-k \theta$ has a local maximum that is not a global maximizer, then locally relaxing the constraints may not be useful, as there may not be any other allocation in the neighborhood of the original allocation that delivers strictly higher value for the objective function.
} 
$i$ will be strictly worse off by reporting type $j$ for all $j<i .^{11}$ Moreover, it is shown that the payoff to type $r$ is continuous in the amount of transfers to lower types, $\epsilon$. Therefore, if $\epsilon$ is chosen sufficiently small, no incentive compatibility constraint is violated and welfare increases at the same time. This is because type $i$ has generated strictly higher surplus and all other types have generated the same surplus. To finance transfers to types $1,2, \ldots, r-1$, the planner levies a lump sum tax on all types so that no IC constraint is affected. Making transfers across agents does not change welfare; therefore, the welfare level is now strictly higher than that in equilibrium.

It becomes clear from this explanation that although the welfare level increases following this cross-subsidization scheme, types $r+1, r+2, \ldots, I$ become worse off relative to the equilibrium allocation. To study constrained Pareto (in)efficiency of equilibrium, consider another financing scheme in which the planner levies lump sum taxes only on types $1,2, \ldots, r$ to finance transfers to types $1,2, \ldots, r-1$. Under this scheme, the net amount of tax that type $r$ has to pay is $\frac{\sum_{j=1}^{r-1} \pi_{j}}{\sum_{j=1}^{r} \pi_{j}} \epsilon$. If the level of improvement in the surplus of type $r$ resulting from the relaxation of the constraints in $P_{r}$ is more than the net amount of tax levied on type $r$, then type $r$ is weakly better off than in equilibrium. Types below $r$ are strictly better off, as they have received $\epsilon-\frac{\sum_{j=1}^{r-1} \pi_{j}}{\sum_{j=1}^{r} \pi_{j}} \epsilon$ net of transfers. Types above $r$ are not affected by these transfers. The new allocation clearly Pareto dominates the equilibrium allocation. It remains to check that the IC constraints for higher-type problems, $P_{j}$ for $j>r$, have not been tightened. This is obviously true because all types $1,2, \ldots, r$ are weakly better off, so they have even less incentive to report higher types. Higher types obviously do not want to report lower types because $\epsilon$ is small and their IC constraints were slack in equilibrium. This result is summarized in the following theorem, for which some notation is needed.

Denote by $J$ the set of types whose market tightness or production level is distorted relative to the first best allocation, $J=\left\{r \in\{1,2, \ldots, I\} \mid U_{r}^{E Q}<U_{r}^{F B}\right\}$. That is, for any $r \in J$, some constraints in $P_{r}$ are binding. For this result, differentiability is assumed whenever needed to calculate the improvement in the payoff of type $r$ following the relaxation of constraints in $P_{r}$. Denote by $\lambda_{j r}$ the Lagrangian multiplier associated with the $\mathrm{IC}_{j r}$ constraint. Denote by $\bar{U}_{r}(\epsilon)$ the value of the objective function for $P_{r}$ when $U_{j}^{E Q}$ in the constraint is replaced by $U_{j}^{E Q}+\epsilon$ for all $j<r$.

Theorem 2 (Constrained Pareto inefficiency of equilibrium). Assume $U_{i}^{F B}>0$ for all $i$. Assume that there exists a type $r \in J$ such that the following holds: (i) Functions $m(),. u_{j}($. and $v_{j}($.$) are continuously differentiable in the neighborhood of the solution to problem P_{r}$ for

\footnotetext{
${ }^{11}$ Lemma 3 is similar to Lemma 1 in GSW, but my claim is stronger. I prove that higher types are strictly worse off if they apply to submarkets designed for lower types. That is, downward IC constraints cannot be binding. The reason that I obtain a stronger result is that I assume strict monotonicity for $v_{i}(a)$ in $i$ for every $a$ with $a \in \bar{A}$, while they assume weak monotonicity. Strict monotonicity holds in all applications that they study and in many economic applications.
} 
all $j \leq r$. (ii) The equilibrium allocation for type $r$ is interior. (iii) $\bar{U}_{r}(\epsilon)$ is a differentiable function of $\epsilon$ in the neighborhood of $\epsilon=0$. (iv) Also

$$
\sum_{j=1}^{r-1} \lambda_{j r}>\left(1+\frac{\pi_{r}}{\sum_{j=1}^{r-1} \pi_{j}}\right)^{-1} .
$$

Then, an implementable allocation exists which Pareto dominates the equilibrium allocation.

The interesting fact about condition (1) is that its LHS, although being endogenous, is independent from the distribution of types, as $P_{i}$ is independent from the distribution. Therefore, the following result is immediately obtained.

Corollary 1. Assume $U_{i}^{F B}>0$ for all $i$, but equilibrium does not achieve the first best, particularly, $U_{r}^{E Q}<U_{r}^{F B}$ for some $r$. Also assume that requirements (i), (ii) and (iii) in

Theorem 2 hold. If $\frac{\sum_{j=1}^{r-1} \pi_{j}}{\pi_{r}}$ is sufficiently small, then the equilibrium allocation is Pareto dominated by another implementable allocation.

Proof. Equilibrium does not achieve the FB for type $r$, so $\lambda_{j r}>0$ for some $j$. Therefore, the LHS of (1) is strictly positive. The result follows immediately.

This result states that if the equilibrium allocation, which is independent of the distribution of types, does not achieve the first best, and if the relative share of lower types in the population is sufficiently small, then the equilibrium is not constrained Pareto efficient. That is, cross-subsidization can make everybody better off. From a practical point of view, this result suggests that the optimal policy intervention (aiming at improving the allocation for all types) may require the policy maker to have precise information about the distribution of types, and a one-fits-all solution is unlikely to be optimal. From a theoretical point of view, this result implies that the GSW equilibrium concept, built on Gale (1996) with off-the-equilibrium-path belief restrictions in the spirit of Cho and Kreps (1987), is susceptible to introducing even a small fraction of "bad" types, as it can make the allocation worse for everybody. A natural step for future work is to either weaken GSW restrictions on the off-the-equilibrium-path beliefs so that equilibrium becomes constrained efficient, or to introduce other equilibrium concepts to recover efficiency. See Lester et al. (2015) as an attempt related to the latter.

\subsection{Constrained Efficient Allocation}

In the last subsection, I obtained some results about inefficiency of equilibrium. In this subsection, I focus on the planner's problem and provide sufficient conditions for the planner to achieve the first best. Later, I characterize the planner's problem under more general conditions. 
We say that $\hat{a} \geq a$ if $\hat{a}^{l} \geq a^{l}$ for all $l \in\{1,2, \ldots, n\}$, that is, if each component of $\hat{a}$ is greater than each component of $a$. Function $g_{i}(a): A \times\{1,2, . ., I\} \rightarrow \mathbb{R}$ has increasing differences in $(a ; i)$ if $g_{i}(\hat{a})-g_{i}(a)$ is weakly increasing in $i$ for $\hat{a} \geq a$. Function $g_{i}(a)$ is super-modular in $a$ if $g_{i}(a)+g_{i}(\hat{a}) \leq g_{i}(a \vee \hat{a})+g_{i}(a \wedge \hat{a})$ for all $a, \hat{a}$ and $i \in\{1,2, . ., I\}$, where the component $l$ in $a \vee \hat{a}(a \wedge \hat{a})$ is equal to $\max \left\{a^{l}, \hat{a}^{l}\right\}\left(\min \left\{a^{l}, \hat{a}^{l}\right\}\right)$. Let also $\Pi_{i} \equiv \sum_{j=i+1}^{I} \pi_{j}$ for $i<I$, and $\Pi_{I} \equiv 0$.

Assumption 2. The following conditions hold:

(i) Monotonicity of $u$ in $i: u_{1}(a) \leq u_{2}(a) \leq \ldots \leq u_{I}(a)$ for all $a \in \bar{A}$.

(ii) $u_{i}(a)$ has increasing differences in $(a ; i)$ for all $a \in \bar{A}$ and $i$.

Assumption 3. $f_{i}(a) \equiv u_{i}(a)+v_{i}(a)$ is super-modular in a and has increasing differences in $(a ; i)$ for all $a \in \bar{A}$ and $i$.

Assumption 4. Either (i) holds or both (ii) and (iii) hold:

(i) Monotonicity of $v$ in $i: v_{1}(a) \leq v_{2}(a) \leq \ldots \leq v_{I}(a)$ for all $a \in \bar{A}$.

(ii) Monotonicity of $f$ in $i: f_{1}(a) \leq f_{2}(a) \leq \ldots \leq f_{I}(a)$ for all $a \in \bar{A}$.

(iii) Sufficient gains from trade for all types:

$$
\sum_{i=1}^{I} \pi_{i}\left[U_{i}^{F B}-m\left(\theta_{i}^{F B}\right)\left(u_{i+1}\left(a_{i}^{F B}\right)-u_{i}\left(a_{i}^{F B}\right)\right) \frac{\Pi_{i}}{\pi_{i}}\right] \geq 0 .
$$

Theorem 3 (Sufficient conditions to achieve the first best). Under Assumptions 2, 3 and 4 , the planner achieves the first best.

Assumption 2(i) simply states that the payoff to higher types is higher than that to lower types for any given level of production. For example, it is less costly for higher types to produce the output. Assumption 2(ii) is equivalent to the single crossing condition, which is also called the Spence-Mirrlees condition, for a broad class of functions. ${ }^{12}$ If $u$ is differentiable (although I do not impose differentiability for this result), this assumption implies that for a given level of production, the marginal payoff to higher types with respect to the level of production of a good $l \in\{1,2, \ldots, n\}$ is higher than that of lower types. The first part of Assumption 3 is simply a standard super-modularity condition requiring that the marginal surplus created by type $i$ with respect to the level of production of good $l_{1}$ is increasing in the level of production of good $l_{2}\left(l_{1} \neq l_{2}\right)$. Assumption 4 requires either one of the two following conditions: For any given level of production, buyers weakly prefer higher types of sellers. Or, the match surplus is higher for higher types, and the maximum possible surplus in this

\footnotetext{
${ }^{12}$ See Milgrom and Shannon (1994) for a full discussion about single crossing condition, increasing differences property and super-modularity, and the relationship between them.
} 
economy, $\sum_{i=1}^{I} \pi_{i} U_{i}^{F B}$, is weakly higher than the total amount of information rents given to sellers, $\sum_{i=1}^{I} \pi_{i}\left(m\left(\theta_{i}^{F B}\right)\left(u_{i+1}\left(a_{i}^{F B}\right)-u_{i}\left(a_{i}^{F B}\right)\right) \frac{\Pi_{i}}{\pi_{i}}\right)$. See Section 3.3.1 for the interpretation of information rents.

The requirements of Theorem 3 are not weaker nor stronger than the requirements of Theorem 1. For example, Assumption 1 requires strict monotonicity of $v_{i}$, while Assumption 4(i) requires only weak monotonicity. On the other hand, Assumption 3(i) requires weak monotonicity of $u_{i}$, while Assumption 1 does not. Furthermore, although Assumptions 1(ii) and 3(ii) are similar, they are not exactly the same. A standard single crossing condition states that the indifference curves of different types must cross only once. The sorting assumption here, which is the same as in GSW, is in a sense a local crossing condition, because it allows $a^{\prime}$ to be greater than $a$ for some $a$ and less than $a$ for other $a$. Moreover, it is in a sense stronger than a single crossing condition, because it states that given any $a$, there exists an $a^{\prime}$ with such a property. ${ }^{13}$

The proof follows a guess-and-verify approach. I first guess that the planner can achieve the first best, then I verify that conditions for feasibility are satisfied. The goal is to find a feasible mechanism in which type $i$ sellers match with probability $m\left(\theta_{i}^{F B}\right)$ and produce $a_{i}^{F B}$. For that, one needs to find a set of transfers that together with $\left(\theta_{i}^{F B}, a_{i}^{F B}\right)$ satisfy IC and other constraints. To find such a set, it is shown that if Assumption 2(i) holds and if transfers are such that all local downward IC constraints are satisfied and are binding, then all IC constraints are satisfied. By local downward (upward) IC constraint, I mean that type $i$ should not gain by reporting type $i-1(i+1)$. Following this construction method, the amount of transfers to the lowest type, $s_{1}^{*}$, determines the amount of transfers for all other types. This set of transfers satisfies all IC constraints if $\left(\theta_{i}^{F B}, a_{i}^{F B}\right)$ is increasing in $i$ and $u$ has increasing differences property in $(a ; i)$, i.e., Assumption 2(ii). See Theorem 7.1 and 7.3 in Fudenberg and Tirole (1991) or Section 3.1 in Laffont and Martimort (2009) for reference.

To show that $\left(\theta_{i}, a_{i}\right)$ is increasing in $i$, note that if $u_{i}+v_{i}$ satisfies Assumption 3, then $a_{i}^{F B} \equiv \arg \max _{a \in \mathbb{A}}\left\{u_{i}(a)+v_{i}(a)\right\}$ is increasing in $i$, i.e., $a_{i}^{F B} \geq a_{i-1}^{F B}$, according to Theorem 5 in Milgrom and Shannon (1994). If $u_{i}+v_{i}$ is increasing in $i$ (Assumption 2(i) together with 4(i), or Assumption 4(ii) alone), then $m(\theta)\left(u_{i}\left(a_{i}^{F B}\right)+v_{i}\left(a_{i}^{F B}\right)\right)-k \theta$ has increasing differences in $(\theta ; i)$. Also, $m(\theta)\left(u_{i}\left(a_{i}^{F B}\right)+v_{i}\left(a_{i}^{F B}\right)\right)-k \theta$ is clearly super-modular in $\theta$ because $\theta$ is one-dimensional. Again, according to Theorem 5 in Milgrom and Shannon (1994), $\arg \max _{\theta}\left\{m(\theta)\left(u_{i}\left(a_{i}^{F B}\right)+v_{i}\left(a_{i}^{F B}\right)\right)-k \theta\right\}$ will be increasing in $i$. Hence, $\left(\theta_{i}^{F B}, a_{i}^{F B}\right)$ is increasing in $i$.

Finally, the planner adjusts $s_{1}^{*}$ such that all types receive a positive payoff. It is interesting

\footnotetext{
${ }^{13}$ In many economic applications, if Assumptions 4(i) and 3 (second part) are satisfied, it is likely that Assumptions 1(i) and 1(ii) are satisfied, respectively. Thus, very loosely speaking, the requirements of Theorem 3 (Assumptions 2, 3 and 4) are likely to be stronger than requirements of Theorem 1 (Assumptions 1(i) and (ii)), although not much can be said about Assumption 1(iii).
} 
to note that under Assumption 4(i), which is independent from the distribution of types, the budget-balance condition is satisfied, so the first best allocation is implementable regardless of the distribution. If Assumption 4(i) fails, then Assumption 4(iii) is sufficient for the budget-balance condition to hold. In future sections, I will make it clear by presenting some applications, the mechanism through which the planner can achieve superior outcomes than the market. Particularly, I indicate in application 2 that Assumptions 2, 3 and 4(i), though seemingly strong, hold in a natural labor market model.

\subsubsection{General Characterization}

Throughout the discussion, I assume Assumptions 2 and 3 are satisfied. If Assumption 4 is also satisfied, then the planner achieves the first best (Theorem 3). Theorem 4 below, which is especially useful if Assumption 4 is not satisfied, provides necessary conditions for the planner's problem. It is usually easy to pin down the solution from these necessary conditions.

Define virtual surplus as

$$
r_{i}^{\rho}(a) \equiv u_{i}(a)+v_{i}(a)-\rho \frac{\Pi_{i}}{\pi_{i}}\left(u_{i+1}(a)-u_{i}(a)\right) \text { for } \rho \in[0,1]
$$

The interpretation of the virtual surplus is that when the planner wants to allocate $\left(\theta_{i}, a_{i}\right)$ to type $i$, incentive compatibility condition requires that $\rho m\left(\theta_{i}\right)\left(u_{i+1}\left(a_{i}\right)-u_{i}\left(a_{i}\right)\right)$ is left for all types above $i$ as information rent. Therefore, the total rent is $\rho m\left(\theta_{i}\right)\left(u_{i+1}\left(a_{i}\right)-\right.$ $\left.u_{i}\left(a_{i}\right)\right) \sum_{j=i+1}^{I} \pi_{j}$. Variable $\rho$ shows the relative strength of the budget-balance condition. If $\rho=0$, then the virtual surplus is exactly equal to the surplus of the match. This is the case only if there are enough resources available to the planner to satisfy BB so that he would not distort the market tightness or production levels. Higher $\rho$ corresponds to conditions under which the constraints are tighter, so the planner needs to leave more rents for higher types by distorting the market tightness or production levels. See Jullien (2000) for a related discussion.

Assumption 5. $r_{i}^{\rho}(a)$ is super-modular in a and has increasing differences in $(a ; i)$ for all $a \in \bar{A}$, for all $i$ and $\rho \in[0,1]$.

Assumption 6. Monotonicity of $r^{\rho}$ in $i: r_{1}^{\rho}(a) \leq r_{2}^{\rho}(a) \leq \ldots \leq r_{I}^{\rho}(a)$ for all $a \in \bar{A}$ and $\rho \in[0,1] .^{14}$

Theorem 4. Suppose Assumptions 2, 5 and 6 hold and all $u_{i}(a), v_{i}(a)$ and $m(\theta)$ are continuously differentiable. Denote the optimal mechanism by $\left\{\left(\theta_{i}^{*}, a_{i}^{*}, s_{i}^{*}\right)\right\}_{i \in\{1,2, \ldots, I\}}$. If $\left(\theta_{i}^{*}, a_{i}^{*}\right)$ is

\footnotetext{
${ }^{14}$ Assumption 5 is stronger than Assumption 3, as the former boils down to the latter for $\rho=0$. Similarly, Assumption 6 is stronger than Assumption 4(ii).
} 
interior for all $i$, then there exists $\zeta^{*} \in \mathbb{R}_{+}$such that the optimal mechanism satisfies:

$$
\begin{gathered}
\frac{\partial \mathcal{L}_{i}\left(\theta_{i}^{*}, a_{i}^{*}, \zeta^{*}\right)}{\partial \theta}=0 \text { and } \frac{\partial \mathcal{L}_{i}\left(\theta_{i}^{*}, a_{i}^{*}, \zeta^{*}\right)}{\partial a^{l}}=0 \text { for all } i \in\{1,2, \ldots, I\} \text { and } l \in\{1,2, \ldots, n\} \\
\sum_{i=1}^{I} \pi_{i} \mathcal{L}_{i}\left(\theta_{i}^{*}, a_{i}^{*}, \zeta^{*}\right) \geq 0 \text { with equality if } \zeta^{*}>0
\end{gathered}
$$

where $\mathcal{L}_{i}(\theta, a, \zeta) \equiv\left(m(\theta)\left(u_{i}(a)+v_{i}(a)\right)-k \theta_{i}\right)(1+\zeta)-\zeta m(\theta)\left(u_{i+1}(a)-u_{i}(a)\right) \frac{\Pi_{i}}{\pi_{i}}$.

The main insight from the proof is that local downward IC constraints and the PC of the lowest type are sufficient to characterize the optimal mechanism even if the first best allocation is not implementable. The proof is somewhat similar to the proof of Theorem 3. However, further work is required because the guess-and-verify approach does not work as the planner may not achieve the first best. Since the planner's problem is hard to handle, as a standard trick in the mechanism design literature, I first try to solve a more relaxed problem. Instead of considering all IC and PC constraints, only local downward IC constraints and PC constraint of the lowest type are taken into account to write a modified budget-balance condition, and then the total amount of surplus in the economy is maximized subject to only this modified constraint. Considering the relaxed problem and forming the Lagrangian, one can easily verify that the two equations in the theorem, are necessary for optimality. Finally, even if the solution is not interior, first-order conditions can be easily adjusted, but I do not report the results for that case here to save space.

\section{Application 1: Asset Market with Lemons}

I have considered a general framework so far. In the following two sections, I study two applications from GSW, characterize the constrained efficient allocation, and compare it with the respective equilibrium allocation. At the end of this section, I elaborate on the inefficiency results and provide some intuition on how and why the planner can increase welfare by using appropriate transfers.

The first application is an asset market with lemons, in the spirit of Akerlof (1970). There are two types of assets, with value $c_{i}$ to the seller and $h_{i}$ to the buyer. Both $c_{i}$ and $h_{i}$ are in terms of a numeraire good. The payoff of a buyer matched with a type $i$ seller is $a h_{i}-p-k$, where $a$ is the probability that the buyer gets the asset from the seller and $p$ is the amount of the numeraire good he produces, i.e., the price that he pays in terms of the numeraire good. The payoff of a type $i$ seller matched with a buyer is $-a c_{i}+p$, where $a$ is the probability that the seller gives the asset to the buyer and $p$ is the amount of the numeraire good he consumes. The buyer's payoff is $-k$ if unmatched. As a special case of the original setting, here: $I=2, n=1, u_{i}(a)=-a c_{i}$ and $v_{i}(a)=a h_{i}$. The matching 
function is $m(\theta)=\min \{1, \theta\}$, that is, the short side of the market matches with certainty. Following GSW, I make the following assumptions:

Assumption 7. In the asset market with lemons,

(i) $0<h_{1}<h_{2}$ and $0<c_{1}<c_{2}$.

(ii) For $i=1,2, c_{i}<b_{i} \equiv h_{i}-k$.

\subsection{Characterization}

Since there are positive gains from trade for both types according to Assumption 7(ii), under complete information, the planner wants both types to match with probability 1 , $\theta_{1}^{F B}=\theta_{2}^{F B}=1$, and also trade with probability $1, a_{1}^{F B}=a_{2}^{F B}=1$. As already discussed, the market with complete information decentralizes the first best allocation. With adverse selection, GSW equilibrium is unique and separating. Different types trade in two different submarkets. In submarket one, price is lower, but probability of matching for sellers is higher compared with submarket two. The market tightness is used for buyers to screen different types. The probability of matching for type two is distorted so that type one would not want to apply to submarket two. The equilibrium allocation is independent of the distribution of types. The second and third columns of Table 1 include the equilibrium outcomes under complete information and adverse selection, respectively. Now let's turn to the constrained efficient allocation.

Proposition 1. Suppose Assumption 7 holds. Then the planner achieves strictly higher welfare than the equilibrium. The optimal mechanism is given by:

$$
\left(\theta_{1}^{*}, a_{1}^{*}, s_{1}^{*}, \theta_{2}^{*}, a_{2}^{*}, s_{2}^{*}\right)=\left\{\begin{array}{ll}
\left(1,1, \pi_{1} b_{1}+\pi_{2} b_{2}, 1,1, \pi_{1} b_{1}+\pi_{2} b_{2}\right) & \text { if } \pi_{1} b_{1}+\pi_{2} b_{2} \geq c_{2} \\
\left(1,1, \frac{\pi_{1} b_{1}\left(c_{2}-c_{1}\right)+\pi_{2} c_{1}\left(c_{2}-b_{2}\right)}{c_{2}-\pi_{1} c_{1}-\pi_{2} b_{2}}, \frac{\pi_{1}\left(b_{1}-c_{1}\right)}{c_{2}-\pi_{1} c_{1}-\pi_{2} b_{2}}, 1,0\right) & \text { if } \pi_{1} b_{1}+\pi_{2} b_{2}<c_{2}
\end{array} .\right.
$$

Finally, if $\pi_{1} c_{1}+\pi_{2} b_{2} \geq c_{2}$, then the equilibrium is Pareto dominated by another implementable allocation.

The first part of this proposition is a special case of Theorem 1. In the second part, to fully characterize the optimal mechanism, two cases are considered separately. If $\pi_{1} b_{1}+\pi_{2} b_{2} \geq c_{2}$, then the planner achieves the first best, for example, through a pooling allocation. This claim is stronger than Theorem 3, because a weaker assumption than Assumption 4 is required. ${ }^{15}$ The allocation implemented by this mechanism, which is given in the fourth column of Table 1 , does not require the planner to actually use any transfers. All he needs to do is to set zero

\footnotetext{
${ }^{15}$ To apply Theorem 3 to this setting, first switch the labels of type one and type two. Now see that Assumption 2 is satisfied. Assumptions 4(iii) is satisfied if $\pi_{2}\left(U_{2}^{F B}-m(1)\left(-c_{1}+c_{2}\right) \frac{\pi_{1}}{\pi_{2}}\right)+\pi_{1} U_{1}^{F B} \geq 0$, which is equivalent to $\pi_{1} b_{1}+\pi_{2} b_{2} \geq c_{2}$. However, for Assumptions 3 and 4(ii) to hold, $h_{2}-c_{2} \leq h_{1}-c_{1}$ is needed.
} 
tax for the pooling price $p=\pi_{1} b_{1}+\pi_{2} b_{2}$ and levy large taxes on other submarkets. This allocation cannot be sustained as an equilibrium, because buyers would have incentives to open a new submarket with a slightly higher price to attract only high-type sellers from the pool, i.e., cream skimming, but then the probability that high-type sellers match would be reduced compared with the first best.

If $\pi_{1} b_{1}+\pi_{2} b_{2}<c_{2}$, type two would receive less than 0 under the pooling allocation, so pooling both types is not feasible. Therefore, the first best is not achievable via a pooling allocation. This is not achievable through any separating allocation either, because if $a_{1}=$ $a_{2}=\theta_{1}=\theta_{2}=1$, then the payment to sellers in both submarkets should be the same to satisfy the IC constraint, but it is already shown that the pooling allocation is not feasible. The constrained efficient allocation in this case is given in the fifth column of Table 1.

GSW show that in the asset market with lemons, if there are no gains from trade only for type one, that is, $b_{1}-c_{1}<0$ and $b_{2}-c_{2}>0$, then the entire market will shut down. I show that in this case, if $\pi_{1} b_{1}+\pi_{2} b_{2}<c_{2}$, then the planner cannot help. See page 65 in the Appendix for the proof, but the intuition is as follows. Type two is not active in the equilibrium, so given the IC constraint of type one, the highest payoff that type two can receive in the market is zero, so type two chooses not to participate in the market. Therefore, both IC constraints are binding as both types receive a zero payoff anyway. The trick that worked in the proof of Theorem 1 is not effective here, because any direct subsidies intended for type one equally attract type two sellers, so type two would also prefer to report to be type one. The policy implication is that if the distortion in the market is so severe that inactivity of one type in equilibrium leads to inactivity of other types, then the planner may not be able to improve the allocation using a budget-balanced policy.

\subsection{Explanation of the Results}

Assume as a thought experiment that the planner begins from the equilibrium allocation and wants to increase welfare. We have already seen that the equilibrium allocation is implementable through zero taxes. In the equilibrium, type one is indifferent between choosing submarket one and submarket two. Although some type two sellers are unmatched in submarket two, buyers do not enter submarket two further, because more entry will make submarket two strictly preferable for type one, leading to entry of type one to submarket two. Nevertheless, matching with type one sellers in submarket two with a positive probability is not worthwhile for buyers given the high price that they need to pay in submarket two.

To increase welfare, the planner increases the net payment to type one, so that the IC constraint of type one for choosing submarket two becomes slack. The planner implements this allocation by subsidizing buyers in submarket one, i.e., $t_{1}<0$. According to buyers' maximization and zero profit condition, buyers in submarket one pay the net amount of $b_{1}$, which is equal to $p_{1}+t_{1}$. But $t_{1}<0$, so $p_{1}>b_{1}$. In other words, taxes are levied on 


\begin{tabular}{|c|c|c|c|c|}
\hline & $\begin{array}{c}\text { Complete } \\
\text { information } \\
(\mathrm{FB})\end{array}$ & Equilibrium & $\begin{array}{c}\text { Constrained } \\
\text { efficient } \\
\text { if } \pi_{1} b_{1}+\pi_{2} b_{2} \geq c_{2}\end{array}$ & $\begin{array}{c}\text { Constrained } \\
\text { efficient } \\
\text { if } \pi_{1} b_{1}+\pi_{2} b_{2}<c_{2}\end{array}$ \\
\hline \hline$a_{1}, a_{2}$ & 1 & 1 & 1 & 1 \\
\hline$\theta_{1}$ & 1 & 1 & 1 & 1 \\
\hline$\theta_{2}$ & 1 & $\frac{b_{1}-c_{1}}{b_{2}-c_{1}}$ & - & $\frac{\pi_{1}\left(b_{1}-c_{1}\right)}{c_{2}-\pi_{1} c_{1}-\pi_{2} b_{2}}$ \\
\hline$p_{1}$ & $b_{1}$ & $b_{1}$ & $\pi_{1} b_{1}+\pi_{2} b_{2}$ & $\frac{\pi_{1} b_{1}\left(c_{2}-c_{1}\right)+\pi_{2} c_{1}\left(c_{2}-b_{2}\right)}{c_{2}-\pi_{1} c_{1}-\pi_{2} b_{2}}$ \\
\hline$p_{2}$ & $b_{2}$ & $b_{2}$ & - & $c_{2}$ \\
\hline$t_{1}$ & - & - & 0 & $-\frac{\pi_{2}\left(b_{2}-c_{2}\right)\left(b_{1}-c_{1}\right)}{c_{2}-\pi_{1} c_{1}-\pi_{2} b_{2}}$ \\
\hline$t_{2}$ & - & - & $b_{2}(\operatorname{large})$ & $\frac{b_{2}-c_{2}}{c_{2}}$ \\
\hline$U_{1}$ & $b_{1}-c_{1}$ & $b_{1}-c_{1}$ & $\pi_{1} b_{1}+\pi_{2} b_{2}-c_{1}$ & $\frac{\pi_{1}\left(b_{1}-c_{1}\right)\left(c_{2}-c_{1}\right)}{c_{2}-\pi_{1} c_{1}-\pi_{2} b_{2}}$ \\
\hline$U_{2}$ & $b_{2}-c_{2}$ & $\frac{b_{1}-c_{1}}{b_{2}-c_{1}}\left(b_{2}-c_{2}\right)$ & $\pi_{1} b_{1}+\pi_{2} b_{2}-c_{2}$ & 0 \\
\hline
\end{tabular}

Table 1: Different allocations in the asset market with lemons. Here, $U_{i}$ denotes the payoff to type $i$ in different allocations, and $t_{i}$ denotes the tax amount levied on buyers in submarket $i$ in the constrained efficient allocation. The tax on other submarkets is sufficiently large and $t_{0}=0$. If $\pi_{1} b_{1}+\pi_{2} b_{2} \geq c_{2}$, the planner achieves the first best through a pooling allocation where both types trade in one submarket with $p=\pi_{1} b_{1}+\pi_{2} b_{2}$.

sellers anyway. As a result, type one strictly prefers submarket one over submarket two following this subsidy. Now more buyers have incentives to enter submarket two to match with previously unmatched sellers of type two. To finance subsidies to trading in submarket one, the planner must tax trading in submarket two. The planner keeps increasing $\left|t_{1}\right|$ and $t_{2}$ until one of the following happens. Either he achieves the first best, which is the case in the pooling allocation where both types trade with the same price and with probability 1 , or the participation constraint of type two sellers binds, i.e., type two sellers receive an exactly zero payoff. The former happens if $\pi_{1} b_{1}+\pi_{2} b_{2} \geq c_{2}$ and the latter happens if $\pi_{1} b_{1}+\pi_{2} b_{2}<c_{2}$.

When $\pi_{1} b_{1}+\pi_{2} b_{2} \geq c_{2}$, if $\pi_{2}$ is sufficiently large, the price in the pooling allocation is sufficiently high so that type two sellers prefer this allocation to equilibrium. This is because the gains that type two sellers receive from the increase in the speed of trading by pooling all types is more than the effective tax that they have to pay. Remember that type two sellers in equilibrium receive $b_{2}$ with probability $\theta_{2}^{E Q}<1$, while they receive $\pi_{1} b_{1}+\pi_{2} b_{2}<b_{2}$ with probability 1 in the constrained efficient allocation. Put differently, type two sellers are forced to pay a high cost in the equilibrium to separate themeselves from type one sellers, so they prefer collectively to cross-subsidize type one sellers if there are few of them, and trade faster instead.

Entry of more buyers into a submarket in directed search environments creates two types 
of externalities on others. First, it decreases the probability of matching for other buyers in that submarket. Second, it increases the probability of matching for sellers in that submarket and consequently their payoff. In the complete information case, the change in the payoff to sellers in one submarket does not affect the payoff to sellers in other submarkets. In fact, the negative externality that entrants impose on other buyers under complete information is exactly offset by the amount of positive externalities that they impose on sellers and therefore, the equilibrium allocation is efficient. Under private information, the change in the payoff to one type of sellers changes the IC constraints that other buyers face in other submarkets, affecting the set of feasible contracts that those buyers can offer to attract other types of sellers. This, in turn, will affect the payoff to other sellers in other submarkets. The buyers in the market economy do not take this effect into account. The planner, in contrast, internalizes these externalities by levying appropriate level of taxes, and therefore, is able to increase welfare.

\section{Application 2: The Rat Race}

In this section I study another application from GSW, the rat race, which was originally discussed in Akerlof (1976). An interesting fact about this application is that the first best is achievable only through a separating allocation, in contrast to the previous example where the first best was achievable through a pooling allocation for some parameter values. More interestingly, the first best is achievable regardless of the distribution of types.

There are two types of workers (as sellers) on one side of the market and firms (as buyers) on the other side. The payoff of a type $i$ worker matched with a firm from $a \in\left[0, a_{\text {max }}\right]$ hours of work and consuming $p$ units of the numeraire good is $p-\phi_{i}(a)$, where $a_{\max }>0$ is sufficiently large. The worker's payoff is 0 if unmatched. The payoff of a firm matched with a type $i$ worker when the worker works for $a$ hours and the firm produces $p$ units of the numeraire good is $v_{i}(a)-p-k$. The firm's payoff is $-k$ if unmatched. As a special case of the original setting, here $I=2, n=1$ and $u_{i}(a)=-\phi_{i}(a)$. Matching function $m(\theta)$ is strictly concave and twice differentiable. I make the following assumptions:

Assumption 8. In the rat race application,

(i) Regularity assumptions on disutility of work: $\phi_{i}$ is differentiable, increasing, strictly convex and $\phi_{i}(0)=\phi_{i}^{\prime}(0)=0$.

(ii) Higher disutility of work for lower types: For all $a \in\left[0, a_{\max }\right], \phi_{1}(a)=\tau \phi_{2}(a)$ where $\tau>1$.

(iii) Regularity assumptions on production function: $v_{i}$ is differentiable, increasing and strictly concave.

(iv) Higher productivity of higher types in average and at the margin: For all $a \in \bar{A} \equiv\{a \in$ $\left.\left[0, a_{\max }\right] \mid \max \left\{v_{1}(a)-\phi_{1}(a), v_{2}(a)-\phi_{2}(a)\right\} \geq \frac{k}{q(0)}\right\}, v_{1}(a)<v_{2}(a)$ and $v_{1}^{\prime}(a) \leq v_{2}^{\prime}(a)$. 
Proposition 2. Under Assumption 8, the optimal mechanism is given by:

$$
\begin{gathered}
\left(\theta_{1}^{*}, a_{1}^{*}, \theta_{2}^{*}, a_{2}^{*}\right)=\left(\theta_{1}^{F B}, a_{1}^{F B}, \theta_{2}^{F B}, a_{2}^{F B}\right), \\
s_{1}^{*}=\pi_{1} U_{1}^{F B}+\pi_{2} U_{2}^{F B}+m\left(\theta_{1}^{F B}\right) \tau \phi_{2}\left(a_{1}^{F B}\right)-\pi_{2}(\tau-1) m\left(\theta_{1}^{F B}\right) \phi_{2}\left(a_{1}^{F B}\right), \\
s_{2}^{*}=\pi_{1} U_{1}^{F B}+\pi_{2} U_{2}^{F B}+m\left(\theta_{2}^{F B}\right) \phi_{2}\left(a_{2}^{F B}\right)+\pi_{1}(\tau-1) m\left(\theta_{1}^{F B}\right) \phi_{2}\left(a_{1}^{F B}\right) .
\end{gathered}
$$

I argue here that if Assumption 8 holds, then Assumption 2, 3 and 4(i) are automatically satisfied, so this result is a special case of Theorem 3. Assumption 2(i) is satisfied because $-\phi_{1}(a)<-\phi_{2}(a)$ for all $a$. Assumption $2(\mathrm{ii})$ is satisfied because $-\phi_{2}(a)-\left(-\phi_{1}(a)\right)$ is increasing in $a$ as $\tau>1$. Assumption 3 is satisfied because $v_{2}(a)-\phi_{2}(a)-\left(v_{1}(a)-\phi_{1}(a)\right)$ is increasing in $a$ and because $a$ is simply one-dimensional. Assumption 4(i) is satisfied because $v_{2}(a) \geq v_{1}(a){ }^{16}$

See the fourth column of Table 2 for the full description of the constrained efficient allocation. When $U_{2}^{F B}-U_{1}^{F B}>(\tau-1) m\left(\theta_{2}^{F B}\right) \phi_{2}\left(a_{2}^{F B}\right)$, then the equilibrium does not achieve the first best. It is simple to check that for type two workers, the probability of finding a match and also hours of work are distorted upward in equilibrium compared with the first best allocation, i.e., $\theta_{2}^{E Q}>\theta_{2}^{F B}$ and $a_{2}^{E Q}>a_{2}^{F B}$. The planner achieves the first best by subsidizing trade in submarket one $\left(t_{1}<0\right)$ and taxing trade in submarket two $\left(t_{2}>0\right)$. By offering this schedule of transfers, allocating the low-type workers a higher wage and the high-type workers a lower wage than their wage under complete information, the planner discourages type one workers from applying to submarket two, thus reducing the cost of private information. $^{17}$

When the equilibrium fails to achieve the first best, GSW propose a pooling allocation that Pareto dominates the equilibrium allocation only if $\pi_{1}$ is sufficiently small, although the proposed allocation does not achieve the first best. The planner achieves the first best here regardless of $\pi_{1}$. Moreover, if $\pi_{1}$ is sufficiently small, then the constrained efficient allocation Pareto dominates the equilibrium allocation (Corollary 2). The point is that when equilibrium does not achieve the first best, cross-subsidization is necessary, but it is not necessarily done by pooling all types.

\section{Relationship with Adverse Selection Literature}

In this section I place my results in the adverse selection literature and compare them with important papers of Rothschild and Stiglitz (1976), Wilson (1977), and particularly Holm-

\footnotetext{
${ }^{16} \mathrm{GSW}$ make similar assumptions, except that they require $v_{1}(a) \leq v_{2}(a)$ (with weak inequality) and do not impose $v_{1}^{\prime}(a) \leq v_{2}^{\prime}(a)$. The former and the latter are used here in Theorems 1 and 3 , respectively.

${ }^{17}$ This result can provide a rationale for minimum wage or maximum compensation laws and regulations. In the context of this model, these laws and regulations discourage low-type workers from applying for positions designed for high types, thus relaxing IC constraints that firms, who try to attract higher-type workers, face. Hence, the contract for high-type workers will be distorted less and efficiency will improve.
} 


\begin{tabular}{|c|c|c|c|}
\hline & Complete information & Equilibrium allocation & Planner's allocation \\
\hline \hline$\theta_{1}$ & $\theta_{1}^{F B}$ & $\theta_{1}^{F B}$ & $\theta_{1}^{F B}$ \\
\hline$\theta_{2}$ & $\theta_{2}^{F B}$ & $\theta_{2}^{E Q}$ & $\theta_{2}^{F B}$ \\
\hline$a_{1}$ & $a_{1}^{F B}$ & $a_{1}^{F B}$ & $a_{1}^{F B}$ \\
\hline$a_{2}$ & $a_{2}^{F B}$ & $a_{2}^{E Q}$ & $a_{2}^{F B}$ \\
\hline$p_{1}$ & $p_{1}^{F B} \equiv v_{1}\left(a_{1}^{F B}\right)-\frac{k}{q\left(\theta_{1}^{F B}\right)}$ & $p_{1}^{F B}$ & $p_{1}^{F B}-t_{1}$ \\
\hline$p_{2}$ & $p_{2}^{F B} \equiv v_{2}\left(a_{2}^{F B}\right)-\frac{k}{q\left(\theta_{2}^{F B}\right)}$ & $v_{2}\left(a_{2}^{E Q}\right)-\frac{k}{q\left(\theta_{2}^{E Q}\right)}$ & $p_{2}^{F B}-t_{2}$ \\
\hline$t_{1}$ & 0 & 0 & $-\frac{\pi_{2}}{m\left(\theta_{1}^{F B}\right)}\left(U_{2}^{F B}-U_{1}^{F B}\right)+\pi_{2}(\tau-1) \phi_{2}\left(a_{1}^{F B}\right)$ \\
\hline$t_{2}$ & 0 & 0 & $\frac{\pi_{1}}{m\left(\theta_{2}^{F B}\right)}\left(U_{2}^{F B}-U_{1}^{F B}\right)-\pi_{1}(\tau-1) \frac{m\left(\theta_{1}^{F B}\right)}{m\left(\theta_{2}^{F B}\right)} \phi_{2}\left(a_{1}^{F B}\right)$ \\
\hline
\end{tabular}

Table 2: Different allocations in the rat race. Here $t_{i}$ denotes the tax amount levied on buyers in the submarket $\left(a_{i}, p_{i}\right)$ in the constrained efficient allocation. The tax on other submarkets is sufficiently large, and $t_{0}=0$.

ström and Myerson (1983) and Maskin and Tirole (1992). I try to keep my notation as close as possible to the last two papers so as to make the comparison simpler. First, I formally define several notions: ex-ante incentive efficient, interim incentive efficient, and ex-post classically efficient allocations introduced by Holmström and Myerson (1983), and Rothchild-Stiglitz-Willson (RSW or least-cost separating) and weakly interim incentive efficient allocations introduced by Maskin and Tirole (1992). Second, using the language of this section, I restate Theorems 1-4, so that my results can be directly comparable with this literature. Finally, I explain what search frictions add to the picture.

There is a finite number of agents in Holmström and Myerson (1983), each with private information, and there are exactly two agents in Maskin and Tirole (1992) with only one having private information. In my paper, I have a continuum of sellers and buyers. To make the comparison possible, I use the following interpretation. Instead of having a continuum of agents, consider an environment with only one seller and one buyer (only for this section). The seller is of type $i$ with probability $\pi_{i}>0$ and has private information about his type. Define $\pi \equiv\left(\pi_{1}, \pi_{2}, \ldots, \pi_{I}\right)$. The buyer has no private information. Let $\delta:\{1,2, \ldots, I\} \rightarrow$ $\mathbb{R}_{+} \times \mathbb{A} \times \mathbb{R}_{+}$denote an allocation. Elements of $\delta$ are simply $(\theta, a, s)$ and $\delta(i) \equiv\left(\theta_{i}, a_{i}, s_{i}\right)$. Let $D$ denote the set of all technologically feasible allocations:

$$
D=\left\{(\theta, a, s) \in \mathbb{R}_{+} \times \mathbb{A} \times \mathbb{R}_{+} \mid \theta \leq M, q(0)\left(v_{i}(a)-s\right) \geq 0 \text { and } u_{i}(a)+s \geq 0 \text { for all } i\right\}
$$

where $M$ is a sufficiently large number. Let $\Delta$ denote the set of all classically feasible allocations: $\Delta=\{\delta:\{1,2, \ldots, I\} \rightarrow D\}$. Let the payoff to the buyer and the seller of type $i$ who reports type $j$ from allocation $\delta$ be, respectively, denoted by:

$$
\begin{gathered}
V(\delta(j), i)=m\left(\theta_{j}\right) v_{i}\left(a_{j}\right)-k \theta_{j}-m\left(\theta_{j}\right) s_{j}, \\
U(\delta(j), i)=m\left(\theta_{j}\right) u_{i}\left(a_{j}\right)+m\left(\theta_{j}\right) s_{j} .
\end{gathered}
$$


Note that search frictions have been incorporated in the payoff function of agents. In a technical sense, matching probability is simply another dimension of the contracting problem and is not different from, for example, hours of work in the labor market application, except that it enters in the payoff function in a certain way. The set of all incentive compatible (IC) allocations is given by:

$$
\Delta^{*}=\{\delta \in \Delta \mid U(\delta(i), i) \geq U(\delta(j), i) \text { for all } i, j\} .
$$

Allocation $\bar{\delta}$ is defined to be ex-ante or interim incentive efficient relative to the prior beliefs $\pi$, i.e., $\bar{\delta} \in \Delta_{A}^{*}(\pi)$ or $\bar{\delta} \in \Delta_{I}^{*}(\pi)$, if it solves

$$
\max _{\delta} \sum \pi_{i} \lambda_{S}(i) U(\delta(i), i)
$$

subject to $\delta \in \Delta^{*}, U(\delta(i), i) \geq 0$ for all $i$ and $\sum \pi_{i} V(\delta(i), i) \geq \sum \pi_{i} V(\bar{\delta}(i), i)$,

with respective measurability restrictions on $\lambda_{S}(i)$ : if $\lambda_{S}(i)$ is independent of $i$, then we get ex-ante incentive efficient allocations, and if it depends on $i$ arbitrarily, then we get interim incentive efficient allocations. Obviously, any ex-ante incentive efficient allocation is interim incentive efficient. Regarding the constraints of the problem, the first one is IC and the second one is seller's participation constraint. ${ }^{18}$ The last one is buyer's ex-ante and also interim participation constraint. This constraint is not type-by-type because the buyer does not know the type of the seller at the ex-ante or interim stage.

Similarly, allocation $\bar{\delta}$ is defined to be ex-post classically efficient, i.e., $\bar{\delta} \in \Delta_{P}$, if it solves

$$
\max _{\delta} \sum \lambda_{S}(i) U(\delta(i), i)
$$

subject to $\delta \in \Delta, U(\delta(i), i) \geq 0$ and $V(\delta(i), i) \geq V(\bar{\delta}(i), i)$ for all $i$

for some positive weights $\lambda_{S}(i)$, where $\lambda_{S}(i)$ can depend on $i$ arbitrarily. ${ }^{19}$

Allocation $\delta^{R S W}$ is defined relative to $\delta_{O}$, with associated payoff $U^{R S W, i}$ to type $i$, to be RSW if for all $i$,

$$
U^{R S W, i} \equiv U\left(\delta^{R S W}(i), i\right)=\max _{\delta} U(\delta(i), i)
$$

\footnotetext{
${ }^{18} \mathrm{I}$ have assumed that the seller cannot commit to participate after learning his type. Otherwise, the seller's participation constraint for ex-ante case would be an average taken over all types, i.e., $\sum \pi_{i} U(\delta(i), i) \geq 0$.

${ }^{19} \mathrm{~A}$ similar formulation of efficiency concepts were first introduced by Holmström and Myerson (1983): Denote by $\Delta_{J}\left(\Delta_{J}^{*}\right)$ the set of allocations that maximizes $W(\delta)=\sum_{i=1}^{I} \pi_{i}\left(\lambda_{B}(i) V(\delta(i), i)+\lambda_{S}(i) U(\delta(i), i)\right)$ over $\Delta\left(\Delta^{*}\right)$ with respective measurability constraints on $\lambda_{B}(i)$ and $\lambda_{S}(i)$ for $J \in\{A, I, P\}$. If $\lambda_{B}(i)$ and $\lambda_{S}(i)$ depend on $i$ arbitrarily, then we obtain ex-post efficient allocations. If $\lambda_{B}(i)$ is independent of $i$, but $\lambda_{S}(i)$ depends on $i$ arbitrarily, then we obtain interim efficient allocations. If both are independent of $i$, then we obtain ex-ante efficient allocations. Unlike their paper, I have incorporated participation constraints at different stages into the definitions of efficiency. If participation constraints are not taken into account, then the following results will immediately follow: $\Delta_{A} \subseteq \Delta_{I} \subseteq \Delta_{P}, \Delta_{A}^{*} \subseteq \Delta_{I}^{*} \subseteq \Delta_{P}^{*}$, and $\Delta_{J} \cap \Delta^{*} \subseteq$ $\Delta_{J}^{*}$ for all $J \in\{A, I, P\}$. See also Myerson (1983).
} 


$$
\text { subject to } \delta \in \Delta^{*} \text {, and } V(\delta(i), i) \geq V\left(\delta_{O}(i), i\right) \text { for all } i,
$$

where, for simplicity and to conform to the formulation in the previous sections, I define $\delta_{O}$ to be no-trade allocation, i.e., $\delta_{O}(i)=(0,0,0)$ for all $i$. Denote the set of all RSW allocations by $\Delta^{R S W}$. Allocation $\bar{\delta}$ is defined to be weakly interim incentive efficient, i.e., $\bar{\delta} \in \Delta_{W I}^{*}$, if it solves

$$
\max _{\delta} \sum \lambda_{S}(i) U(\delta(i), i)
$$

subject to $\delta \in \Delta^{*}$, and $V(\delta(i), i) \geq V(\bar{\delta}(i), i)$ for all $i$

for some positive weights $\lambda_{S}(i)$.

\subsection{Results in the Language of Adverse Selection Literature}

It is straightforward to show that the set of first best allocations defined in Section 3.1 is exactly equal to the set of ex-post classically efficient allocations defined in this section, and that the set of equilibrium allocations in GSW is exactly equal to the set of RSW allocations in this section (within the class of deterministic allocations, as I do not allow for lotteries). ${ }^{20}$ Furthermore, the set of constrained efficient allocations as defined in Section 2 coincides exactly with the set of ex-ante incentive efficient allocations in this section. Finally, the set of implementable allocations that are not Pareto dominated by another implementable allocation coincides exactly with the set of interim incentive efficient allocations in this section. To summarize, all efficiency and equilibrium concepts defined in previous sections have completely standard counterparts in this literature if matching probability is appropriately incorporated into the payoff function of buyers and sellers.

Now that all needed material has been introduced, Theorems 1-4 in this paper are stated in the language of this section for the class of quasi-linear two-agent economies in which one agent has private information about his type:

$\Delta^{R S W} \cap \Delta_{P}=\emptyset$ plus other requirements specified in Theorem $1 \Rightarrow \Delta^{R S W} \cap \Delta_{A}^{*}(\pi)=\emptyset$ for all $\pi$ $\Delta^{R S W} \cap \Delta_{P}=\emptyset$ plus other requirements specified in Theorem $2 \Rightarrow \Delta^{R S W} \cap \Delta_{I}^{*}(\pi)=\emptyset$ for some $\pi$ requirements specified in Theorem $3 \Rightarrow \Delta_{A}^{*}(\pi) \subseteq \Delta_{P}$ $\delta \in \Delta_{A}^{*}(\pi)$ plus other requirements specified in Theorem $4 \Rightarrow \delta$ satisfies Equations (2), (3)

\footnotetext{
${ }^{20}$ For characterization of GSW equilibrium, look at $P_{i}$ in Section 3.2 or Propositions 1-3 in GSW for more details. Characterization method of Proposition 2 in Maskin and Tirole (1992) is very similar to GSW: Start from the lowest type and assign them the best possible allocation subject to the participation constraints, and then solve higher types' problems subject to the participation constraints and incentive compatibility of some lower types. There are two minor differences: First, their contract space is only two-dimensional and thus more limited than GSW. Second, they only consider the incentive compatibility of the adjacent lower type, but in GSW, all downward IC constraints should be taken into account.
} 
Remember that Theorems 1 and 2 require that the GSW equilibrium does not achieve the first best. In the language of this section, it means that the set of RSW allocations have an empty intersection with ex-post efficient allocations. Interestingly, all requirements of Theorems 1 and 2 on the LHS of the arrow are independent of the distribution of types. Hence, Theorem 1 (2) basically states that any RSW allocation is inefficient for all (some) type distributions in the respective sense. Theorem 3 states that under certain conditions, any ex-ante incentive efficient allocation is ex-post efficient; that is, adverse selection is basically irrelevant. Theorem 4 provides some necessary conditions for ex-ante incentive efficient allocations.

To the best of my knowledge, Theorems 1 and 2 have not been established by Maskin and Tirole (1992) or other papers in the literature in their generality: no restriction on the number of types or dimensions of contract space (except being finite) and no restriction on the concavity of the payoff or matching functions. Not only are these results more general, but they also extend the basic intuitions regarding adverse selection environments to those with search frictions.

Maskin and Tirole (1992) characterize RSW allocation and show that any RSW allocation is weakly interim incentive efficient and that any such allocation is interim incentive efficient relative to some beliefs. They imply that any RSW allocation is interim incentive efficient relative to some beliefs. However, Theorem 2 here states that, again in the language of this section, any RSW allocation is not interim incentive efficient relative to some beliefs. Furthermore, they characterize the equilibrium of informed principal game, in which an informed principal offers a mechanism to an uninformed agent. They show that the equilibrium is unique if and only if RSW allocation is interim incentive efficient. As a byproduct of my analysis, Theorem 2 provides sufficient conditions for the equilibrium of the informed principal game to be not unique. ${ }^{21}$

\subsection{Role of Search Frictions}

First, as pointed out by GSW, introducing search frictions with capacity constraints solves the equilibrium non-existence problem that is present in standard adverse selection models such as Rothschild and Stiglitz (1976). ${ }^{22}$ This is probably the most important role of search frictions. Second, search allows us to model several phenomena, which are otherwise hard

\footnotetext{
${ }^{21}$ For the informed principal problem with common values, see Myerson (1983), Cella (2008), Severinov (2008), and Balkenborg and Makris (2015).

${ }^{22}$ Similar to GSW, Gale (1996), Inderst and Wambach (2001) and Inderst and Wambach (2002) use capacity constraints to guarantee equilibrium existence. Other papers such as Wilson (1977), Miyazaki (1977), Riley (1979), and more recently Lester et al. (2015), have modified the game to guarantee existence. Some others have used the competitive markets framework in which agents take the price and composition of types as given not only for on- but also off-the-equilibrium-path prices. See Gale (1992), Dubey and Geanakoplos (2002) and Dubey et al. (2005).
} 
to study with standard economic theory, such as liquidity in financial and other markets, unemployment and the time it takes for a house to sell.

Why do we use a directed search model as opposed to, perhaps, a random search model? Directed search models have some advantages: First, directed search allows economic agents to compete in offering terms of trade to attract agents on the other side of the market. This feature is absent in random search models where agents are more passive in attracting a trade partner. As shown in the literature already cited, equilibrium in directed search models with complete information is usually efficient, thanks to the competition between agents in posting contracts. Second, directed search may be more realistic in many contexts, such as wage posting in the labor markets, than random search. Third, directed search is more suitable for handling heterogeneity. For example, it is difficult to generate different trading delays for heterogeneous agents in financial markets or heterogeneous extensive (firms entry) and intensive (number of hires) margins in labor markets using a random search model, but it is easy to do so using a directed search model. ${ }^{23}$

Which results in this paper are solely due to search frictions? Except equilibrium existence, which is guaranteed by search frictions (and perhaps more precisely by search frictions and capacity constraints), it is possible to incorporate the dependence of the payoff functions of agents to search technology into modified payoff functions for buyers and sellers, as in this section, and work with them. In that case, matching probability is simply another dimension of contract space, and my results will be easily comparable with other adverse selection models, regardless of whether they have search frictions or not. In fact, this allows me to draw a perfect comparison between my results and other classic results in this literature, and I can clearly show that GSW equilibrium is equivalent to RSW or least-cost separating allocation.

\section{Asset Market with a Continuous Type Space}

In Section 4, the optimal tax, the tax schedule that implements the constrained efficient allocation, requires high-price assets to be taxed and low-price assets to be subsidized. An interesting question is whether the optimal tax is generally monotone in the price of assets. To study this question, the model of Section 4 is extended to a continuous type space. Studying this extension allows me to consider cases in which the value of assets to sellers does not have the same order as the value of assets to buyers. Moreover, various policy

${ }^{23}$ Also, directed search can feature block recursively (Menzio and Shi (2011), Menzio et al. (2013) and Shi (2009)), which proves to be convenient in tracking the evolution of the distribution in dynamic models with heterogeneous agents. 
questions can be addressed about the optimal taxation in the asset markets. ${ }^{24}$ The setting in this section can be seen as a static version of Chang (2014) (or a static version of Guerrieri and Shimer (2014a) with only one asset and some minor adjustments), so I study constrained efficiency in their environments using the analysis in this section. Fortunately, the main ideas regarding equilibrium and constrained efficiency are captured in this static model. ${ }^{25}$

The main ideas discussed in previous sections are used in this section as well, and all main results go through, but the mathematical tools used in the continuous case are different. Some definitions need to be introduced again (counterparts of Definitions 2 and 3), but for the sake of brevity, I only define implementable and constrained efficient allocations here and relegate the discussion of many details to the Appendix.

\subsection{Environment}

There is a continuum of measure one of heterogeneous sellers indexed by $z \in Z \equiv\left[z_{L}, z_{H}\right] \subset$ $\mathbb{R}$, with $F(z)$ denoting the measure of sellers with types below $z$. $F$ is continuously differentiable and strictly increasing in $z$ and $F^{\prime}$ is its derivative. Type $z$ is seller's private information. Similar to the original setting, buyers' and sellers' payoffs are quasi-linear in the numeraire good. A buyer's payoff who enters the market and matches with a type $z$ is $h(z)-p-k$, where $p \in \mathbb{R}$ denotes the amount of the numeraire good that he produces, $h(z)$ denotes the value of the asset to the buyer and $k$ is the entry cost, all in terms of the numeraire good. His payoff is $-k$ if unmatched. The payoff of a type $z$ seller matched with a buyer is $p-c(z)$, where $p \in \mathbb{R}$ denotes the amount of the numeraire good that he consumes and $c(z)$ is the value of the asset to the seller in terms of the muneraire good. His payoff is 0 if unmatched. Functions $h: Z \rightarrow \mathbb{R}$ and $c: Z \rightarrow \mathbb{R}$ are twice continuously differentiable. Matching function $m($.$) is increasing, strictly concave and twice differentiable.$

As a benchmark, I characterize the complete information allocation or first best here, exactly following the discussion in Section 3: $U^{F B}(z)=\max _{\theta}\{m(\theta)(h(z)-c(z))-k \theta\}$, and $\theta^{F B}(z) \equiv \arg \max _{\theta}\{m(\theta)(h(z)-c(z))-k \theta\}$, so

$$
m^{\prime}\left(\theta^{F B}(z)\right)(h(z)-c(z))=k
$$

\footnotetext{
${ }^{24}$ I could do the same exercise with a discrete type space with more than two types, but the technical analysis with a continuous type space is simpler.

25 In a dynamic setting, the planner would have some inter-temporal considerations, because the distribution of types does not necessarily remain the same over time, as some types match more quickly than others and exit the market. The interesting tradeoff is whether the planner wants low-type sellers to find a match early and exit the market, or he wants all sellers to exit the market with approximately the same rate all the way to the end. The analysis of the dynamic setting is beyond the scope of this paper. Since the equilibrium allocation is distribution-free, the equilibrium analysis in the dynamic case is much easier than the analysis of the planner's problem in the dynamic case. However, if one assumes in the dynamic setting that when sellers sell their assets, an offspring is born and endowed with a new asset of the same quality, the same results can be obtained in the dynamic setting, too, as the distribution remains constant.
} 
for both the planner and the market economy with complete information. I assume for simplicity that there are positive gains from trade for all types, i.e., $U^{F B}(z)>0$ for all $z$. Similarly to the discrete type space, it turns out that all types will be active both in equilibrium and in the constrained efficient allocation.

\subsection{Definition of the Planner's Problem}

Let an allocation, $\{G, \mathcal{P}, \Theta, \mu\}$, be a measure $G$ on the set of all possible prices, $\mathbb{P} \equiv \mathbb{R}_{+}$, with support $\mathcal{P}$, a tightness function, $\Theta: \mathbb{P} \rightarrow[0, \infty]$, and a conditional density function of buyers' beliefs regarding the type of sellers who would apply to any price, $\mu(z \mid p): \mathbb{P} \times Z \rightarrow[0,1]$. Let a tax function, $t: \mathbb{P} \rightarrow \mathbb{R}$, denote the amount of tax levied on buyers at each submarket conditional on trade, and finally, let $t_{0} \in \mathbb{R}_{+}$denote the amount of the numeraire good to be transferred to all sellers in a lump sum way.

Definition 5. An allocation, $\{G, \mathcal{P}, \Theta, \mu\}$, is implementable through policy $\left\{t, t_{0}\right\}$ if the following conditions are satisfied:

(i) Buyers' profit maximization and free entry

For any $p \in \mathbb{P}$,

$$
q(\Theta(p))\left(\int h(z) \mu(z \mid p) d z-p-t(p)\right) \leq k
$$

with equality if $p \in \mathcal{P}$.

(ii) Sellers' optimal search

Let $U(z)=\max \left\{0, \max _{p^{\prime} \in \mathcal{P}}\left\{m\left(\Theta\left(p^{\prime}\right)\right)\left(p^{\prime}-c(z)\right)\right\}\right\}+t_{0}$ and $U(z)=t_{0}$ if $\mathcal{P}=\emptyset$. Then, for any $p \in \mathbb{P}$ and $z, U(z) \geq m(\Theta(p))(p-c(z))+t_{0}$ with equality if $\Theta(p)<\infty$ and $\mu(z \mid p)>0$. Moreover, if $p-c(z)<0$, either $\Theta(p)=\infty$ or $\mu(z \mid p)>0$.

(iii) Feasibility or market clearing For all $z, \int_{\mathcal{P}} \frac{\mu(z \mid p)}{\Theta(p)} d G(p) \leq F^{\prime}(z)$, with equality if $U(z)>t_{0}$.

(iv) Planner's budget-balance condition

$$
\int_{\mathcal{P}} q(\Theta(p)) t(p) d G(p) \geq t_{0} \cdot{ }^{26}
$$

Using the revelation principle, I assume without loss of generality that sellers are allocated to different submarkets through a direct mechanism to characterize IC schemes. Let $\left\{\tilde{\theta}(),. \tilde{p}(),. \tilde{t}(),. \tilde{t}_{0}\right\}$ be a direct mechanism where $\tilde{\theta}: Z \rightarrow \mathbb{R}_{+}, \tilde{p}: Z \rightarrow \mathbb{R}_{+}, \tilde{t}: Z \rightarrow \mathbb{R}$ and $\tilde{t}_{0} \in \mathbb{R}_{+}$. Whenever $\sim$ is used for a variable, it indicates that a direct mechanism

\footnotetext{
${ }^{26} \mathrm{I}$ assume in this section that there are positive gains from trade for all types, so $t_{0}$ is redundant in this section and is set to 0 as it can be incorporated into $t($.$) . I included t_{0}$ so that the model can be easily used even if there are no gains from trade for some types.
} 
is concerned. In a direct mechanism if a type $z$ seller reports $\hat{z}$, his payoff is given by: $U(z, \hat{z}) \equiv\{m(\tilde{\theta}(\hat{z}))(\tilde{p}(\hat{z})-c(z))\}+\tilde{t}_{0}$. Therefore, the payoff to type $z$ is given by:

$$
U(z)=\max _{\hat{z}} U(z, \hat{z})
$$

Incentive compatibility (IC) constraint implies that $\hat{z}=z$.

An equilibrium allocation, $\left\{G^{E Q}, \mathcal{P}^{E Q}, \Theta^{E Q}, \mu^{E Q}\right\}$, in the market economy is basically an implementable allocation through the policy of zero taxes, $t(p)=0$ for all $p \in \mathbb{P}$ and $t_{0}=0$. Denote by $\left\{\tilde{\theta}^{E Q}(),. \tilde{p}^{E Q}().\right\}$ the direct mechanism associated with the equilibrium in the market economy, and denote by $U^{E Q}($.$) the equilibrium payoff. { }^{27}$ Denote by $\left\{\tilde{\theta}^{*}(),. \tilde{p}^{*}(),. \tilde{t}^{*}(),. \tilde{t}_{0}^{*}\right\}$ the direct mechanism associated with the constrained efficient allocation. In the Appendix, I state and prove the analogous of Lemma 1 to show that there is no loss of generality in working with direct mechanisms.

Definition 6. A constrained efficient allocation is an implementable allocation that maximizes welfare among all implementable allocations. That is, a constrained efficient allocation solves the following problem:

$$
\max _{\{G, \mathcal{P}, \Theta, \mu\},\left\{t, t_{0}\right\}} \int U(z) d F(z)
$$

subject to $\{G, \mathcal{P}, \Theta, \mu\}$ is implementable through $\left\{t, t_{0}\right\}$,

where $U(z)$ is defined in part (ii) of Definition 5.

\subsection{Constrained Efficiency versus Equilibrium}

Define $H_{1}(z) \equiv-\int_{z}^{z_{H}} m\left(\theta^{F B}(\hat{z})\right) h^{\prime}(\hat{z}) d \hat{z}$ and $H_{2}(z) \equiv \int_{z_{L}}^{z} m\left(\theta^{F B}(\hat{z})\right) h^{\prime}(\hat{z}) d \hat{z}$. An allocation is constrained Pareto efficient if there does not exist another allocation that is implementable and gives all sellers a weakly higher payoff and a strictly positive measure of sellers a strictly higher payoff. The results in this section are continuous-type analogous of Theorems 2 and 3. The proof ideas and the intuition are similar. Theorem 1 is easy to extend so I do not repeat it here. A counterpart of Theorem 4 is available upon request.

Proposition 3. Suppose $c^{\prime}(z)>0$ and $U^{F B}(z)>0$ for all $z$.

(i) If $h^{\prime}(z) \leq 0$, then the planner achieves the first best.

(ii) If $h^{\prime}(z) \leq 0$ and

$$
\bar{H}_{1} \equiv \int H_{1}(z) d F(z) \geq \int\left(m\left(\tilde{\theta}^{E Q}(z)\right)-m\left(\theta^{F B}(z)\right)\right) c^{\prime}(z) d z
$$

\footnotetext{
${ }^{27}$ Taxes are zero in the market economy, so they were simply eliminated from the description of the mechanism.
} 
then the constrained efficient allocation Pareto dominates the equilibrium allocation.

(iii) Assume $h^{\prime}(z) \leq c^{\prime}(z)$. The planner achieves the first best if and only if

$$
\bar{H}_{2} \equiv \int H_{2}(z) d F(z) \geq \int m\left(\theta^{F B}(z)\right) c^{\prime}(z) d z-U^{F B}\left(z_{L}\right)
$$

(iv) If $0 \leq h^{\prime}(z) \leq c^{\prime}(z)$ and

$$
\bar{H}_{2} \geq \int\left(m\left(\theta^{F B}(z)\right)-m\left(\tilde{\theta}^{E Q}(z)\right)\right) c^{\prime}(z) d z,
$$

then the constrained efficient allocation Pareto dominates the equilibrium allocation.

It is shown in the proof of Proposition 3 that all types trade in submarkets with different market tightness; therefore, the allocation is separating and $\tilde{p}^{*}(z), \tilde{\theta}^{*}(z)$ and $\tilde{t}^{*}(z)$ are just functions (as opposed to correspondences) of $z$, and are given by:

$$
\begin{gathered}
\tilde{\theta}^{*}(z)=\theta^{F B}(z) \text { for all } z \\
\tilde{p}^{*}(z)=c(z)+\frac{U\left(z_{H}\right)+\int_{z}^{z_{H}} m\left(\tilde{\theta}^{*}\left(z_{0}\right)\right) c^{\prime}\left(z_{0}\right) d z_{0}}{m\left(\tilde{\theta}^{*}(z)\right)} \text { for all } z,
\end{gathered}
$$

where $U\left(z_{H}\right)=\int\left[m\left(\tilde{\theta}^{*}(z)\right)(h(z)-c(z))-k \tilde{\theta}^{*}(z)-m\left(\tilde{\theta}^{*}(z)\right) c^{\prime}(z) \frac{F(z)}{F^{\prime}(z)}\right] d F(z)$, and

$$
\tilde{t}^{*}(z)=h(z)-\tilde{p}^{*}(z)-\frac{k}{q\left(\tilde{\theta}^{*}(z)\right)} \text { for all } z \text {. }
$$

Some comments are in order regarding conditions (6), (7) and (8). First, the RHS of these conditions is independent of the distribution of types and all effects of the distribution are captured in the LHS, so the cost of assets for sellers does not have direct interactions with the distribution of types. That is, the average cost to the sellers is irrelevant as long as the average value of the respective $H_{i}$ (not $h$ ) over the population is sufficiently high. Second, it is easy to see that (7) is stronger than (8). Third, (7) is equivalent to $\int\left[m\left(\theta^{F B}(z)\right)(h(z)-c(z)-\right.$ $\left.\left.c^{\prime}(z) \frac{F(z)}{F^{\prime}(z)}\right)-k \theta^{F B}(z)\right] F^{\prime}(z) d z \geq 0$, or

$$
\int\left(\frac{h(z)-c(z)}{c^{\prime}(z)} \eta\left(\theta^{F B}(z)\right)-\frac{F(z)}{F^{\prime}(z)}\right) m\left(\theta^{F B}(z)\right) c^{\prime}(z) F^{\prime}(z) d z \geq 0,
$$

where $\eta(\theta) \equiv-\frac{\theta q^{\prime}(\theta)}{q(\theta)}$. See the derivation in the proof of Proposition 3(iii) in the Appendix. For the latter inequality to hold, it is sufficient that the terms inside the brackets are positive for all $z$. Fourth, (6) and (8) are hard to verify, because they have been stated in terms of $\tilde{\theta}^{E Q}($.$) , for which the closed-form solution is not generally available. Rather, it is available in$ the form of a differential equation, as shown in (14). In Example 3 in the Appendix, I adopt a specific matching function, solve for $\tilde{\theta}^{E Q}($.$) , and give explicit conditions equivalent to (6)$ and (8). Fifth, technically speaking, part (i) of the proposition is redundant, as it is implied 
by part (iii). This is because if $h^{\prime}(z) \leq 0$, then both $h^{\prime}(z) \leq c^{\prime}(z)$ and (7) are satisfied. I included part (i) to have a clear characterization.

Here is an outline of the proof for parts (i) and (iii) regarding how the first best can be achieved. This proof is similar to that of Theorem 3. The assumption that $c^{\prime}()>$. is kept throughout this section. To characterize incentive compatible allocations, note that the seller's payoff function can be written as $-m(\theta) c(z)$ plus some transfers. This function satisfies the single crossing condition in $(\theta, z)$ (Theorem 7.3 in Fudenberg and Tirole (1991)). Therefore, $\tilde{\theta}(z)$ being decreasing in $z$ implies that there exists a set of transfers to sellers that satisfies IC constraints. Now assume $h^{\prime}(z) \leq 0$ for all $z$ or $h^{\prime}(z) \leq c^{\prime}(z)$ for all $z$. In either case, $\theta^{F B}(z) \equiv m^{\prime-1}\left(\frac{k}{h(z)-c(z)}\right)$ is decreasing in $z$ according to (4). Therefore, if $\tilde{\theta}^{*}(z)=\theta^{F B}(z)$ for all $z$, one can find such transfers. The envelope theorem can then be applied to (5) to obtain:

$$
U^{*}(z)-U^{*}\left(z_{H}\right)=-\int_{z_{H}}^{z} m\left(\tilde{\theta}^{*}(z)\right) c^{\prime}(z) d z,
$$

which together with (5) and the fact that $\hat{z}=z$ yields to (9).

As mentioned earlier, the environment here is basically the static version of Chang (2014), so I take the characterization of equilibrium from her paper.

Proposition 4 (Equivalent to Proposition 1 in Chang (2014)). Suppose $c^{\prime}(z)>0, h^{\prime}(z) \geq 0$ and $U^{F B}(z)>0$ for all $z$. Given the policy of zero taxes, a unique implementable allocation (equilibrium) exists. The equilibrium is separating. The market tightness solves the differential equation (14). The initial condition and prices are given by $\tilde{\theta}^{E Q}\left(z_{L}\right)=\theta^{F B}\left(z_{L}\right)$ and $\tilde{p}^{E Q}(z)=h(z)-\frac{k}{q\left(\tilde{\theta}^{E Q}(z)\right)}$.

Similar to the discrete-type case, whenever I use equilibrium without any reference, I mean an allocation that is implementable through the policy of zero taxes. The IC constraints faced by agents in the market economy are the same as those faced by the planner; therefore, (5) can be used to describe IC constraints in equilibrium too. However, the amount of transfers that each type receives is different in equilibrium than the constrained efficient allocation, because they are pinned down by the free-entry condition absent of any cross-subsidization in equilibrium.

Following GSW, Chang shows that the equilibrium under Assumption $c^{\prime}()>$.0 and $h^{\prime}() \geq$.0 is separating, so free entry implies that $\tilde{p}^{E Q}(z)=h(z)-\frac{k}{q\left(\tilde{\theta}^{E Q}(z)\right)}$ for all $z$. Therefore, the payoff to type $z$ in the market economy, denoted by $U^{E Q}(z)$, is calculated as follows:

$$
U^{E Q}(z)=\max _{\hat{z}}\left\{m\left(\tilde{\theta}^{E Q}(\hat{z})\right)(h(\hat{z})-c(z))-k \tilde{\theta}^{E Q}(\hat{z})\right\},
$$

where the objective function is the payoff to type $z$ if he reports type $\hat{z}$. FOC with respect to $\hat{z}$ (together with the assumption of differentiability of $\tilde{\theta}(z)$ almost everywhere) yields

$$
\left[m^{\prime}\left(\tilde{\theta}^{E Q}(z)\right)(h(z)-c(z))-k\right] \frac{d \tilde{\theta}^{E Q}(z)}{d z}+m\left(\tilde{\theta}^{E Q}(z)\right) h^{\prime}(z)=0
$$




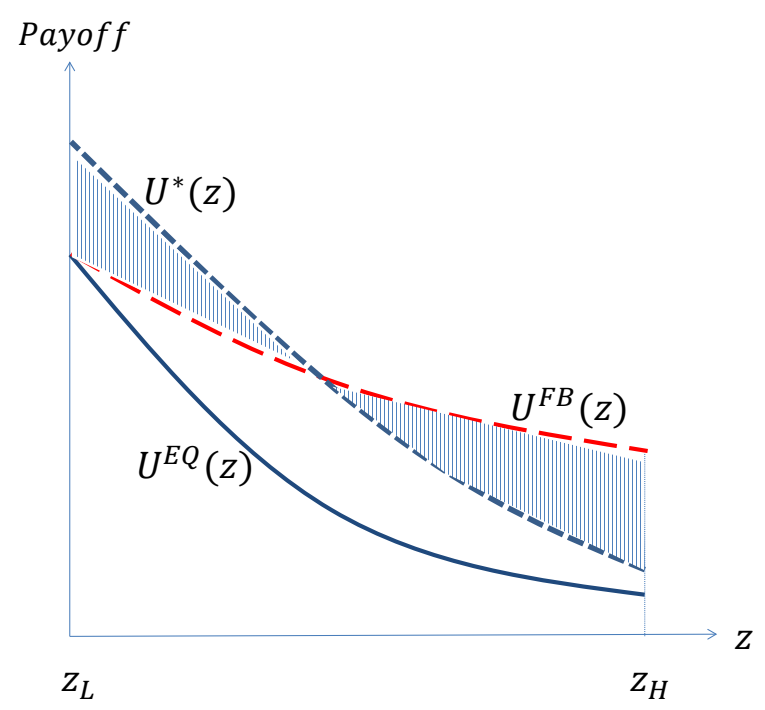

Figure 1: Schematic diagram for sellers' payoffs in different allocations under requirements of Proposition 3 (iv).

where I used the fact that at the solution, $\hat{z}=z$ because of IC. With respect to the initial condition, roughly speaking, the market delivers the complete information payoff to the type that has the most incentive to deviate. For example, when $h^{\prime} \geq 0$, the lowest type has the most incentive to deviate, so the market tightness for this type is set to the complete information level, i.e., $\tilde{\theta}^{E Q}\left(z_{L}\right)=\theta^{F B}\left(z_{L}\right)$.

Now more intuition about Proposition 3, especially part (iv), can be provided. In Figure 1 , the sellers' payoffs in different allocations are illustrated for the case that (8) is satisfied and that $0 \leq h^{\prime}()<.c^{\prime}($.$) . The slope of payoffs in the constrained efficient allocation and$ equilibrium is pinned down by (12), where $\tilde{\theta}^{*}($.$) is replaced by \tilde{\theta}^{E Q}($.$) for the equilibrium$ allocation. The market tightness in the equilibrium is distorted downward, i.e., $\tilde{\theta}^{E Q}($. $\tilde{\theta}^{*}(.) \equiv \theta^{F B}(z)$ by $(14)$, so $U^{*}(z)$ is steeper than $U^{E Q}(z)$. As a result, in order for the constrained efficient allocation to Pareto dominate the equilibrium allocation, the necessary and sufficient condition is that the payoff to the highest type should be weakly higher in the former than the latter. This gives a lower bound for the amount of the payment to the highest type, and the slope of $U^{*}(z)$ determines the payoff to other types. To finance transfers to low-type sellers, the distribution should satisfy (8) so that that the weighted average of the two dashed areas in Figure 1 are the same.

\subsubsection{Disagreement in the Ranking of Assets Between Buyers and Sellers or Two-dimensional Private Information}

Chang (2014) extends her analysis to allow for two-dimensional private information. (See 


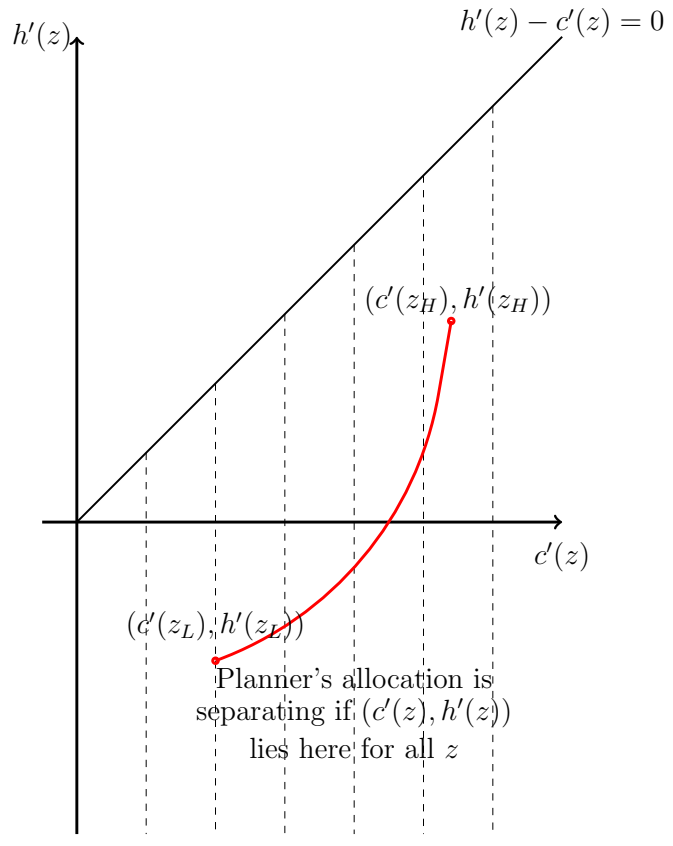

(a)

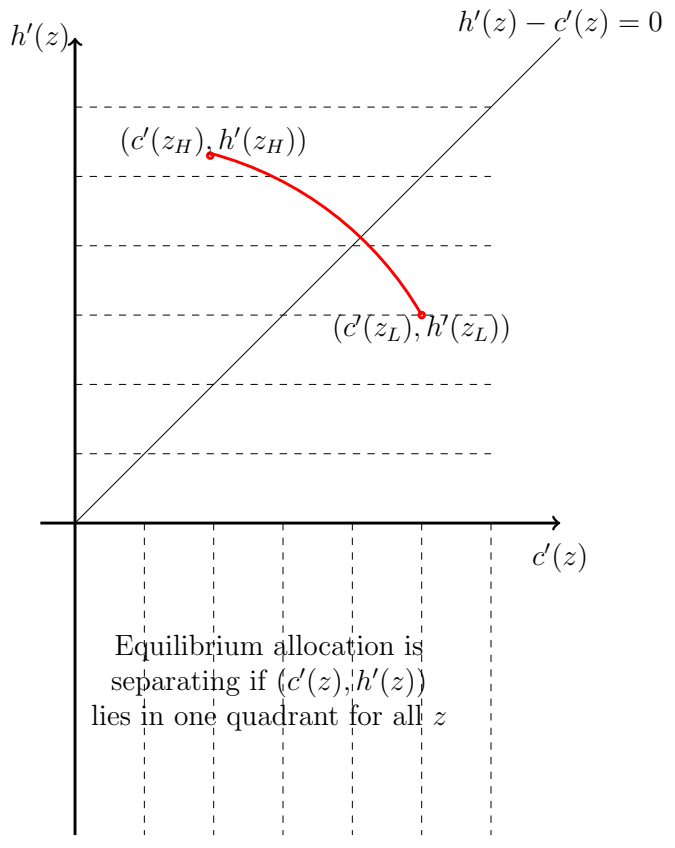

(b)

Figure 2: Assume (7) is satisfied. In (a), $h$ is not monotone in $z$, so the equilibrium will involve some pooling. However, since $h^{\prime}()-.c^{\prime}() \leq$.0 , the planner achieves the first best through a separating allocation (Proposition 3(iii)). In (b), equilibrium is separating because $h^{\prime}() \geq$.0 . However, $h-c$ is not decreasing, so the planner cannot achieve the first best and the constrained efficient allocation may not be separating. 
also Guerrieri and Shimer (2014b) for a closely related model.) What is relevant to this discussion is that following this extension, function $h$ may have a strict local maximum and, therefore, full separation of types in the market is not possible (Proposition 4 in Chang (2014)). Using the same argument and keeping the assumption $c^{\prime}(z)>0$ fixed, it can be shown more generally that if $h$ is not monotone, then full separation of types is not possible. Characterization of the planner's problem in the present paper, in contrast, shows that under $h^{\prime}(z) \leq c^{\prime}(z)$ and also (7), even if $h$ is not monotone, then the constrained efficient allocation is separating. This case is depicted in Figure 2(a) where $h^{\prime}(z)$ is drawn in terms of $c^{\prime}(z)$ for all $z$.

Suppose now that $h^{\prime}(z)-c^{\prime}(z) \leq 0$ is violated for some $z$. For example, $h-c$ has one local minimum, but $h^{\prime}(z) \geq 0$ and $c^{\prime}(z)>0$ both hold, as depicted in Figure 2(b). The equilibrium in this case is separating. The constrained efficient allocation, in contrast, may involve pooling of types, because the monotonicity constraint, that $\tilde{\theta}^{*}(z)$ should be decreasing in $z$, may not be satisfied through any separating allocation. Solving explicitly for the planner's allocation in this case does not give us new insights, so I skip it. The main point is that pooling of types occurs under different conditions in the planner's allocation and the equilibrium allocation. Therefore, drawing conclusions about efficiency of allocation by simply considering whether it is pooling or separating is misleading. There are cases in which the equilibrium allocation is pooling while the efficient allocation is separating and vice versa.

\subsection{Examples of Optimal Taxation}

Two examples are presented in this section to compare the first best (FB), equilibrium (EQ) and constrained efficient $\left(^{*}\right)$ allocations, to identify the types who should be taxed and the ones who should be subsidized, and to examine whether or not taxes are monotone in the type of sellers or in the price of assets.

Example 1. Model parameters: $m(\theta)=1-e^{-\theta}, Z=[1,2], c(z)=z, \quad h(z)=0.5(z-1)^{2}+4$, $k=1.85$, and $F($.$) is uniform.$

Here, $c^{\prime}>0, h^{\prime} \geq 0$ and $h^{\prime}-c^{\prime} \leq 0$. It is easy to check that Proposition 3(iii) applies, so the market tightness for the constrained efficient allocation is given by $\tilde{\theta}^{*}(z)=$ $\theta^{F B}(z)=m^{\prime-1}\left(\frac{k}{h(z)-c(z)}\right)=\ln \left(\frac{h(z)-c(z)}{k}\right)$, and $\tilde{p}^{*}(z)$ and $\tilde{t}^{*}(z)$ are given by (9) and (10). The net payment that buyers make in the constrained efficient allocation, $\tilde{p}^{*}(z)+\tilde{t}^{*}(z)$, is equal to $p^{F B}(z) \equiv h(z)-\frac{k}{q\left(\theta^{F B}(z)\right)}$. For the equilibrium allocation, $\tilde{\theta}^{E Q}(z)$ is given by (14): $\left[\exp \left(-\tilde{\theta}^{E Q}(z)\right)(h(z)-c(z))-k\right] \frac{d \tilde{\theta}^{E Q}(z)}{d z}+\left(1-\exp \left(-\tilde{\theta}^{E Q}(z)\right)\right) h^{\prime}(z)=0$, with the initial condition $\tilde{\theta}^{E Q}(1)=\theta^{F B}(1)$ and $\tilde{\theta}^{E Q}(z)$ being decreasing in $z$. The price that buyers pay in equilibrium is $\tilde{p}^{E Q}(z)=h(z)-\frac{k}{q\left(\tilde{\theta}^{E Q}(z)\right)}$. 
Figure 3 illustrates the first best, equilibrium and constrained efficient allocations for Example 1. Here, $\tilde{\theta}^{E Q}(.) \leq \theta^{F B}($.$) . Market tightness is basically the tool that buyers in the$ market economy use to screen high-type sellers. Low-type sellers prefer to sell their assets more quickly, because they do not want to get stuck with their "lemons." Consequently, $\tilde{p}^{E Q}(z)$ is generally greater than $p^{F B}(z) \equiv h(z)-\frac{k}{q\left(\theta^{F B}(z)\right.}$, the price that buyers pay in the market with complete information. Also, $\tilde{p}^{*}($.$) is higher for lower types and lower for higher$ types compared with $p^{F B}($.$) . Since the market tightness is the same in the first best and$ constrained efficient allocation, the price that buyers should pay is the same in both cases so that buyers' zero profit condition is satisfied. ${ }^{28}$ On the other hand, $\tilde{p}^{*}$ is the payment that sellers should receive in the constrained efficient allocation. Therefore, the amount of tax that buyers should pay, $\tilde{t}^{*}($.$) , is just equal to the difference, p^{F B}()-.\tilde{p}^{*}($.$) .$

Next, I study another example in which $h$ is not monotone and, therefore, separation of types in equilibrium is not possible, as explained in the last subsection.

Example 2. Model parameters: $m(\theta)=1-e^{-\theta}, Z=[0,2], c(z)=z, \quad h(z)=0.5(z-1)^{2}+4$, $k=1.85$, and $F($.$) is uniform.$

Proposition 3(iii) applies, so the market tightness at the constrained efficient allocation is similarly given by $\tilde{\theta}^{*}(z)=\theta^{F B}(z)=m^{\prime-1}\left(\frac{h(z)-c(z)}{k}\right)=\ln \left(\frac{h(z)-c(z)}{k}\right)$. According to Proposition 5 in Chang (2014), I construct one semi-pooling equilibrium in which types $z \in[0,1)$ trade in a pool with a low price but with high probability. Types $z \in(1,2]$ trade in separating submarkets. Type $z=1$ is indifferent between the pool and one of the separating submarkets. She calls this situation fire sale of assets, in which many low-type sellers and some high-type sellers who need liquidity sell their assets with a lower price but very quickly. Prices and taxes are calculated similarly, as explained in Example 1.

Figure 4 is similar to Figure 3 for parameters in Example 2. Market tightness in the constrained efficient allocation is the same as that in the first best. Market tightness in equilibrium is higher than that in the first best for types $z \in[0,1)$ and is less for other types. In Figure 5, the payoff to sellers of different assets is depicted for both Examples 1 and 2.

In the upper left (right) graph of Figure $6, \tilde{t}^{*}(z)$ is drawn in terms of $z$ (in terms of $\tilde{p}^{*}(z)$ ). An interesting fact here is that in both examples, even in Example 1 in which buyers and sellers agree on the ranking of assets as $h$ and $c$ are both increasing, the amount of tax levied on buyers is neither monotone in the type of sellers that buyers meet, nor in the price paid to the sellers. The following lemma provides sufficient conditions for non-monotonicity of

\footnotetext{
${ }^{28} \mathrm{As}$ seen in this figure, the price range in the constrained efficient allocation is smaller than that in the equilibrium. Similarly to the rat race application, this result can provide intuition why price cap and price floor in this market may be welfare enhancing. These policies are basically the same as levying large taxes on very high and very low prices, thus reducing the incentives of low-type sellers to go to the markets designed for higher types.
} 
sale tax schedule if buyers and sellers agree on the ranking of assets. If they don't, deriving sufficient conditions for non-monotonicity is even simpler.

Lemma 2. Assume $0<c^{\prime}($.$) and 0 \leq h^{\prime}(.) \leq c^{\prime}($.$) . Also assume (7) holds, then$

$$
\frac{d \tilde{t}^{*}}{d z}=h^{\prime}(z)+\frac{\left(m^{\prime}\left(\theta^{F B}(z)\right)\right)^{2}}{m\left(\theta^{F B}(z)\right) m^{\prime \prime}\left(\theta^{F B}(z)\right)} \frac{\tilde{t}^{*}(z)}{h(z)-c(z)}\left(h^{\prime}(z)-c^{\prime}(z)\right) .
$$

If $h^{\prime}\left(z_{L}\right)=0$ and $0<h^{\prime}($.$) for a strictly positive measure of sellers, then the optimal sales$ tax schedule is not monotone in the price of assets. Specifically, $\left.\frac{d t^{*}(p)}{d p}\right|_{p=\tilde{p}^{*}\left(z_{L}\right)}<0$ and $\left.\frac{d t^{*}(p)}{d p}\right|_{p=\tilde{p}^{*}\left(z_{0}\right)}>0$ for some $z_{0} \in\left(z_{L}, z_{H}\right]$.

Changes in $\tilde{t}^{*}(z)$ are not only influenced by changes in $h(z)$ but also by another term that has the same sign as $\tilde{t}^{*}(z)$. Therefore, when the first term above is sufficiently small, the second term may dominate the first term and $\tilde{t}^{*}(z)$ may become decreasing. More generally and intuitively, the free-entry condition can be written as follows, if the constrained efficient allocation is separating:

$$
\tilde{t}^{*}(z)=h(z)-\underbrace{\frac{k}{q\left(\tilde{\theta}^{*}(z)\right)}}_{\text {decreasing }}-\underbrace{\tilde{p}^{*}(z)}_{\text {increasing }} .
$$

The term $\frac{k}{q\left(\tilde{\theta}^{*}(z)\right)}$ is decreasing in $z$ because $\tilde{\theta}^{*}(z)$ is decreasing in $z$. Also, $\tilde{p}^{*}($.$) is increasing$ (formally proved in Lemma 7 in the Appendix), as high-type sellers should be compensated for selling with lower probability. Hence, $\tilde{t}^{*}(z)$ may not be generally monotone in $z$.

\subsection{Sales Tax and Entry Tax}

Implementation of a non-monotone tax schedule is difficult in a practical sense, as it requires the planner to have precise information about the distribution of types. Although it is usually assumed in the literature, including in this paper, that the planner has such information, one ideally wants to reduce the dependence of what the planner should do on the details of the economy.

Entry tax is introduced in this subsection, so buyers will be subject to two types of taxes: sales tax, which is conditional on trade as before, and entry tax, which is conditional on entry to each submarket. The definition of implementable allocation should be slightly modified to include both types of taxes. See the Appendix for the details. I show in the following proposition that, in general, any feasible mechanism can be implemented by both a decreasing entry tax and an increasing sales tax in the price of assets. ${ }^{29}$

\footnotetext{
${ }^{29}$ If the entry tax for a submarket is less than $-k$, then buyers pay this negative tax, i.e., receive a positive subsidy of $\tilde{t}_{e}+k$, and then do not participate in the matching stage that delivers them a strictly negative payoff. Therefore, another constraint that should be added to the definition of implementable allocation is $k+\tilde{t_{e}}(z) \geq 0$. See Definition 10 in the Appendix.
} 
Proposition 5 (Implementation of the direct mechanism with monotone entry and sales tax). Take any feasible mechanism in which all types receive a strictly positive payoff, and in which the market tightness allocated to different types is all different. Then there exists an associated implementable allocation with monotone tax schedules in the price of assets, decreasing entry tax and increasing sales tax, such that all types receive the same payoff as their payoff in the feasible mechanism.

The idea to design a monotone $\tilde{t}^{*}(z)$ is to add an entry tax for each submarket, $\tilde{t}_{e}^{*}(z)$, so the free-entry condition can be written as follows:

$$
\tilde{t}^{*}(z)=h(z)-\frac{k+\tilde{t}_{e}^{*}(z)}{q(\tilde{\theta}(z))}-\tilde{p}^{*}(z) .
$$

If ${\tilde{t_{e}}}^{*}(z)$ is constructed to be decreasing sufficiently fast in $z$, then the effect of $\frac{k+\tilde{t_{e}}(z)}{q(\tilde{\theta}(z))}$ dominates the effect of $\tilde{p}^{*}(z)$, and $\tilde{t}^{*}(z)$ becomes increasing in $z$. The following corollary is implied directly by Proposition 5 together with the equivalent of Lemma 1 in the continuoustype space.

Corollary 2. Take an optimal mechanism. Under requirements of Proposition 5, there exists an associated constrained efficient allocation such that all types receive exactly the same payoff as in the mechanism, and the associated entry tax and sales tax are, respectively, decreasing and increasing in the price of assets.

A schedule of monotone sales and entry tax, which implements the first best allocation, is depicted for both examples in Figure 7. 

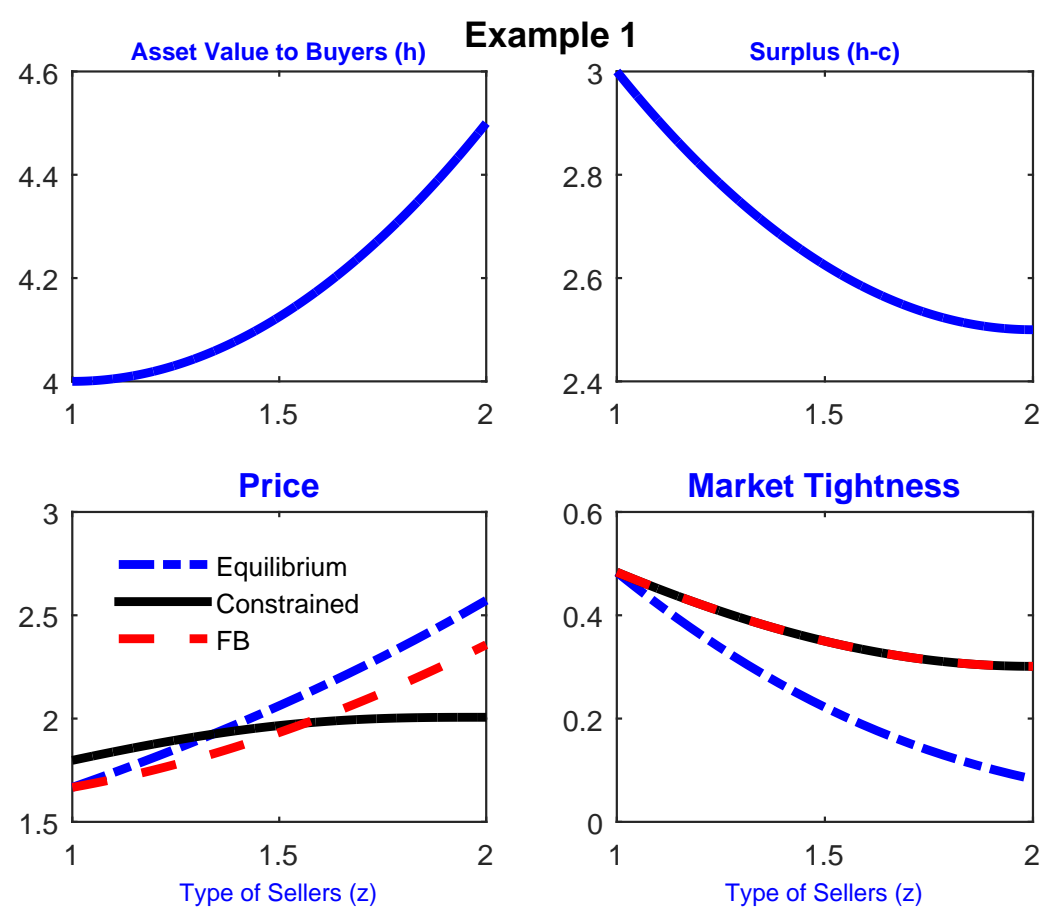

Figure 3: Model parameters are defined in Example 1. In the upper left (right) graph, the value of type $z$ asset to buyers (surplus from the match) is depicted. In the lower left (right) graph, the price that sellers receive (the market tightness) in the first best, constrained efficient and equilibrium allocation is depicted.
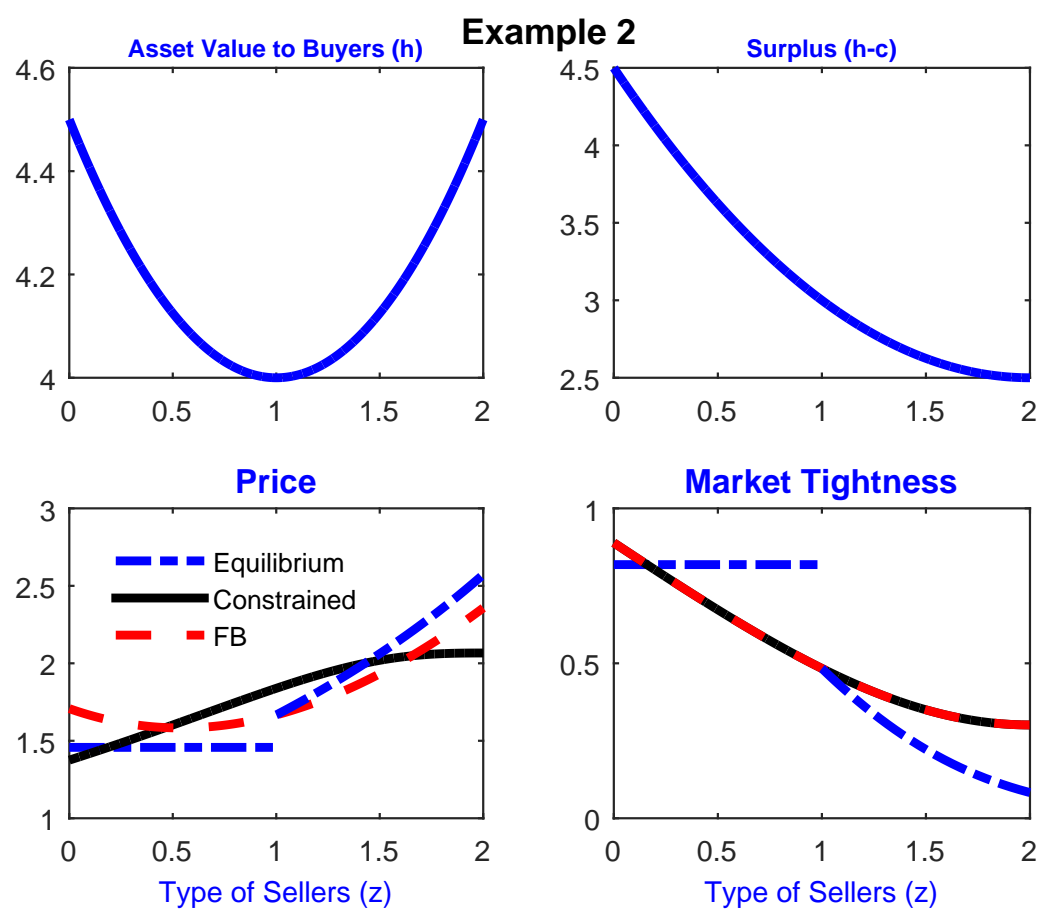

Figure 4: This figure is similar to Figure 3 but with model parameters defined in Example 2. 

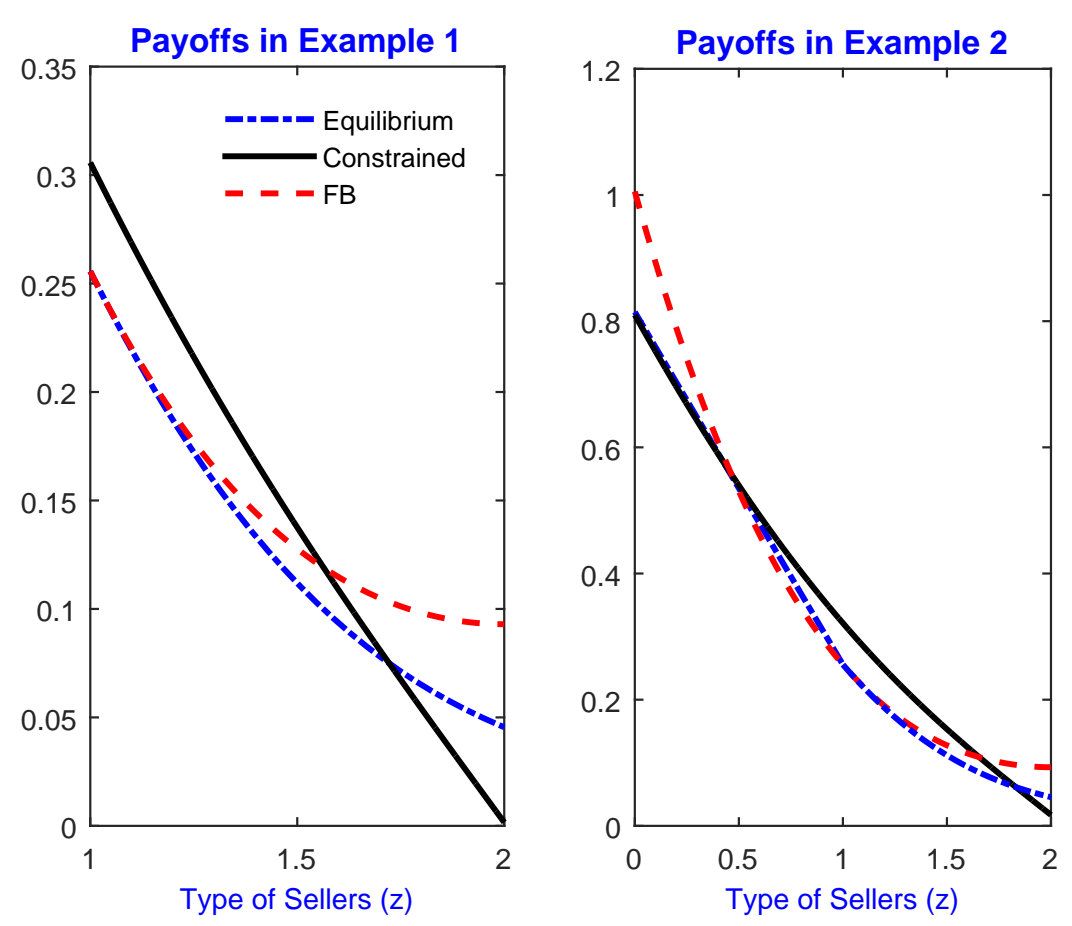

Figure 5: Model parameters are defined in Example 1 (2) for the left (right) graph. The expected payoff to sellers in the first best, constrained efficient and equilibrium allocation is depicted.
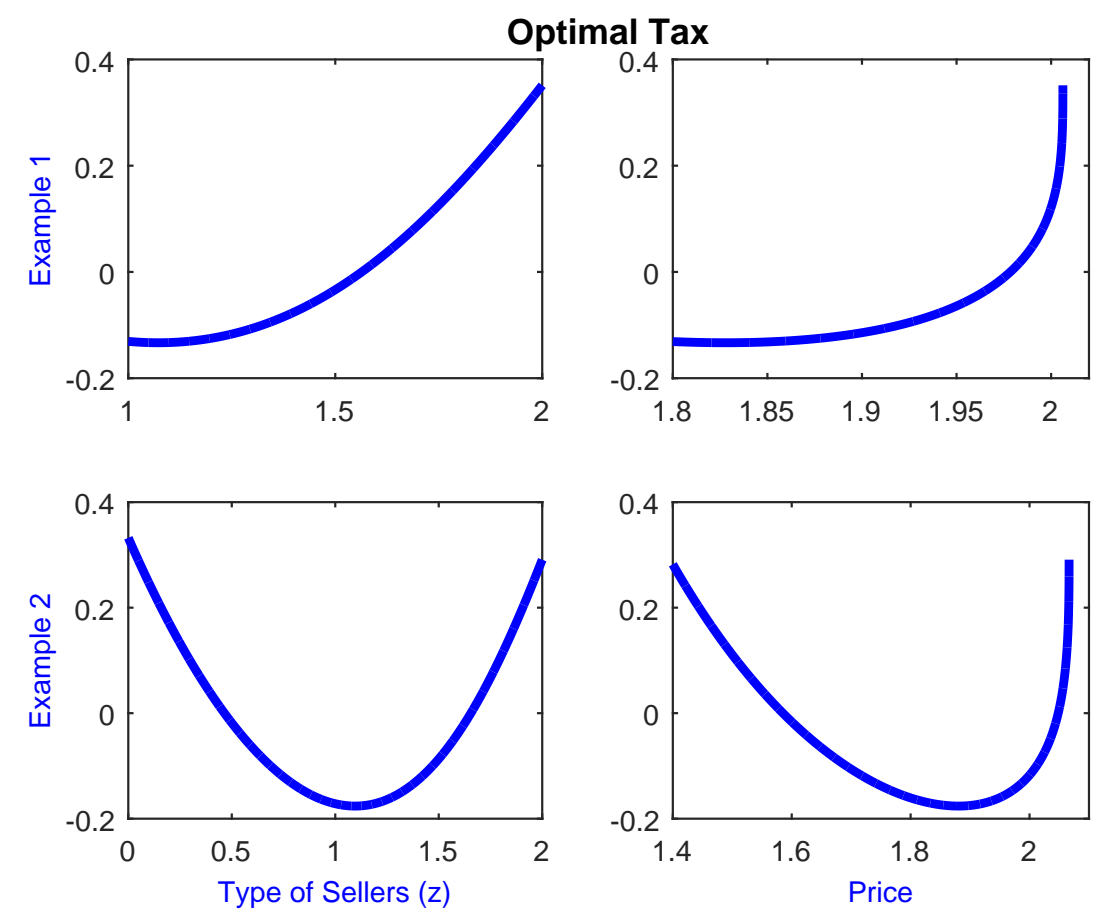

Figure 6: The optimal sales tax schedule is non-monotone in the type or price of assets in both Examples 1 and 2. Specifically in Example 1, the optimal tax has a strict global minimum at $z=1.07$. 

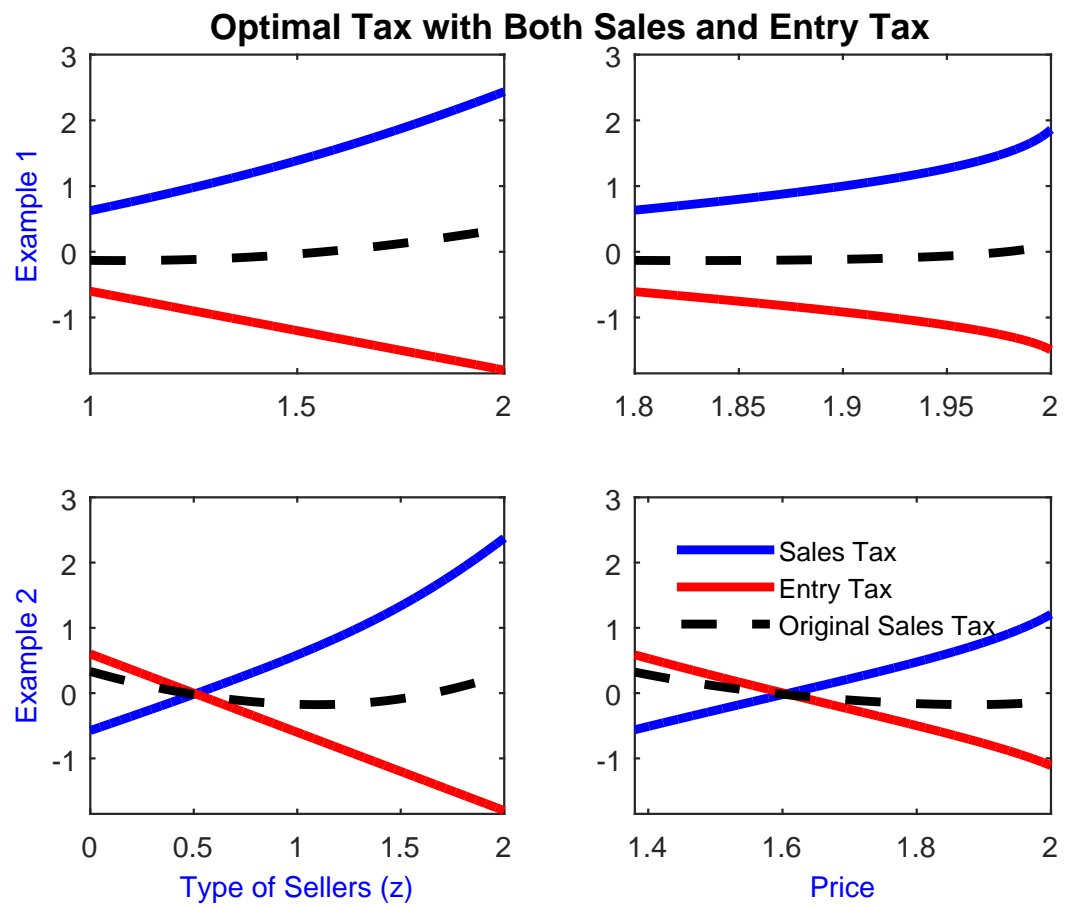

Figure 7: When entry tax is introduced, it can be designed so that both entry tax and sales tax become monotone in the type of sellers or price of assets in both Examples 1 and 2. 


\section{Conclusion}

I characterized the constrained efficient allocation in an environment with adverse selection and directed search. Under similar assumptions that GSW make to characterize the unique equilibrium, the planner can achieve strictly higher welfare than equilibrium if equilibrium fails to achieve the first best. Moreover, if the weight of low types in the population is relatively low, then the equilibrium allocation is Pareto dominated by another implementable allocation. Under some conditions, the planner can even achieve the first best. These results highlight the role of cross-subsidization between different submarkets in such an environment.

In the market economy, the buyers do not take into account the effects of their entry on the set of feasible submarkets available to buyers who want to attract other types of sellers. Entry of one more buyer to a submarket changes the payoff to sellers in that submarket and, through incentive compatibility constraints, changes the set of feasible contracts that buyers can offer in other submarkets, and eventually changes the payoff to sellers in other submarkets. The planner takes this externality into account and is therefore able to increase welfare by imposing appropriate taxes and subsidies.

Guerrieri and Shimer (2014a) use a similar framework to analyze the effects of asset purchase and subsidy programs, such as Troubled Asset Relief Program in 2008 or the PublicPrivate Investment Program for Legacy Assets in 2009. They show that a sales tax schedule monotone in the asset prices increases the price and sale probability of all assets. They do not study the optimal policy, nor do they consider budget-balanced policies. My results show that a monotone sales tax schedule is not generally welfare-maximizing. However, if the planner uses entry tax as well as sales tax, then monotone tax schedules (increasing sales tax and decreasing entry tax) can be found to implement the constrained efficient allocation.

There are many important related research questions that can be considered for future work. For example, if instead of bilateral meetings discussed in this paper, one considers many-on-one meetings, i.e., allowing several sellers to meet with a buyer so that sellers face some competition after meeting a buyer, does it induce sellers to reveal their types in a less costly manner, and importantly, does the equilibrium remain constrained inefficient? Another area for research is to investigate the shape of the optimal tax schedule in more stylized models of asset, insurance, labor and housing markets. This should include relevant details such as common and private values, multi-dimensional heterogeneity, dynamics, possibility of resale, and even symmetric uncertainty shared by buyers and sellers about the value or resale value of the good or asset. Even characterizing the optimal tax schedule in these applications within a certain class of tax functions, such as increasing and convex functions, would be interesting. Finally, modifying the GSW restrictions on the off-the-equilibrium-path beliefs or looking for other equilibrium concepts so that the constrained efficient allocation can be maintained as an equilibrium allocation would be other promising areas for research. 


\section{Appendix}

\section{Differences Between My Environment and GSW}

There are two minor differences between GSW and my environment. The first is that I impose quasi-linearity on the payoff functions, while they do not. I do so to ensure that my results do not come from concavity of the utility functions, and also to make the analysis slightly simpler, as the transfers can be substituted out from the planner's problem by using the budget balance condition. Without quasi-linearity, two changes should be applied to the definition of feasible mechanism (Definition 3). First, the mechanism should have two types of payments to each type of seller: one payment conditional on meeting and one unconditional payment. Here, because of quasi-linearity, I captured the expected payment only in one term, $s_{i}$, without losing any generality. Second, the buyers' participation constraint should be separated from the planner's budget-balance condition as in Definition 1. By applying these two changes, a modified version of Lemma 1 will continue to hold.

The second difference is that I do not allow the planner to use lotteries. By lotteries, I mean that the planner allocates type $i$ sellers to different 3-tuples, $(\theta, a, s)$ and $\left(\theta^{\prime}, a^{\prime}, s^{\prime}\right)$, with positive probabilities, where these 3-tuples may deliver type $i$ sellers different payoffs. In contrast, GSW allow for lotteries on $(a, p)$. However, it does not make their problem a convex one (despite the fact that lotteries are typically used for convexification). The reason is that in the set of problems used to characterize the equilibrium in their paper, both the objective function and the constraint set include $\theta$, and allowing lotteries only on $(a, p)$ does not generally make the constraint set convex or the objective function (quasi-)concave. For the planner, however, allowing for lotteries on both $\theta$ and $(a, p)$ (or equivalently on $(\theta, a, s)$ ) makes sense, as the goal is to characterize the best outcome that can be achieved. It can also make the planner's problem a convex one. The problem, in that case, is that the comparison between the market allocation and the planner's allocation may not be fair, as too much power, lotteries on both $\theta$ and $(a, p)$, has been given to the planner while the market can use lotteries only on $(a, p)$. In short, allowing for lotteries only on $(a, p)$ does not convexify GSW's problem nor the planner's problem.

On the other hand, it seems arbitrary if the planner is allowed to use lotteries along only one dimension, $(a, p)$, and not along the other, $\theta$. That would make the analysis a bit more general at the cost of making the proofs more complicated and less intuitve. For this reason, I decided not to allow for lotteries on $\theta$ nor $(a, p)$ for both the market and the planner, to make the exposition and proofs more intuitive and to have a fair comparison between the market and planner's allocation, without losing any major insights. All results will continue to hold even without quasi-linearity or if lotteries on $(a, p)$ are allowed for both the market and the planner. 


\section{Proofs of Sections 2 and 3}

Before I discuss the results, note that the budget-balance condition in the optimal mechanism is always binding. Otherwise, one can increase all $s_{i}$ by an identical small amount so that all other conditions continue to be met, but welfare strictly increases.

Proof of Lemma 1. Given the feasible mechanism $\left\{\left(\theta_{i}, a_{i}, s_{i}\right)\right\}_{i \in\{1,2, \ldots, I\}}$, an allocation is constructed, shown to be implementable for a proposed policy, and shown to deliver the same welfare as the mechanism. Define $N$ to be the set of all types who match with a strictly positive probability in the direct mechanism, that is, $N \equiv\left\{i \mid \theta_{i}>0\right\}$. Now I construct the following objects:

$$
\begin{gathered}
y_{r_{i}}=\left(a_{i}, \frac{s_{i}-t_{0}}{m\left(\theta_{i}\right)}\right), \Theta\left(y_{r_{i}}\right)=\theta_{i}, \gamma_{i}\left(y_{r_{i}}\right)=1, \lambda\left(\left\{y_{r_{i}}\right\}\right)=\pi_{i} \theta_{i} \text { for all } i \in N, \mathcal{Y}=\left\{y_{r_{i}} \mid i \in N\right\}, \\
t\left(y_{r_{i}}\right)=v_{i}\left(a_{i}\right)-\frac{s_{i}-t_{0}}{m\left(\theta_{i}\right)}-\frac{k}{q\left(\theta_{i}\right)} \text { for all } i \in N, \text { and } t_{0}=\left\{\begin{array}{ll}
s_{i} & \text { if } \exists i \text { such that } \theta_{i}=0 \\
0 & \text { otherwise }
\end{array} .\right.
\end{gathered}
$$

For any other submarket, i.e., $y \notin \mathcal{Y}$, define $K(y)=\left\{j \mid u_{j}(a)+p>0\right\}$ to denote the set of types who would receive a strictly positive payoff by applying to $y$. If $K(y) \neq \emptyset$ and $\min _{j \in K(y)}\left\{\frac{U_{j}}{u_{j}(a)+p}\right\} \leq \bar{m} \equiv \lim _{\theta \rightarrow \infty} m(\theta)$, then set $\Theta(y)$ such that $m(\Theta(y))=\min _{j \in K(y)}\left\{\frac{U_{j}}{u_{j}(a)+p}\right\}$. If the latter equation holds for several $\Theta(y)$, then pick the smallest one. If it holds for no real number, or if $K(y)=\emptyset$ or $\min _{j \in K(y)}\left\{\frac{U_{j}}{u_{j}(a)+p}\right\}>\bar{m}$, then set $\Theta(y)=\infty$. To define the composition function for $y \notin \mathcal{Y}$, define $n_{o}=\min \left\{\arg \min _{j \in K(y)}\left\{\frac{U_{j}}{u_{j}(a)+p}\right\}\right\}$ and set $\gamma_{n_{o}}(y)=1$. If $K(y)=\emptyset$, then $\Gamma(y)$ can be chosen arbitrarily, so, for example, set $\gamma_{1}(y)=1$. Also for $y \notin \mathcal{Y}$, set $t(y)=\max \left\{0, \max _{i} v_{i}(a)-p\right\}$.

If there exist $i$ and $j \neq i$ such that $y_{r_{i}}=y_{r_{j}}$, assuming without loss of generality that $\theta_{i} \leq \theta_{j}$, then keep only $y_{r_{i}}$ and remove $y_{r_{j}}$ from $\mathcal{Y}$ and let $\gamma_{i}\left(y_{r_{i}}\right)=\frac{\pi_{i}}{\pi_{i}+\pi_{j}}, \gamma_{j}\left(y_{r_{i}}\right)=\frac{\pi_{j}}{\pi_{i}+\pi_{j}}$, $\lambda\left(\left\{y_{r_{i}}\right\}\right)=\left(\pi_{i}+\pi_{j}\right) \theta_{i}$ and $t\left(y_{r_{i}}\right)=\frac{\pi_{i} v_{i}\left(a_{i}\right)+\pi_{j} v_{j}\left(a_{i}\right)}{\pi_{i}+\pi_{j}}-\frac{s_{i}-t_{0}}{m\left(\theta_{i}\right)}-\frac{k}{q\left(\theta_{i}\right)} \cdot{ }^{30}$ Now I show that if $y_{r_{i}}=y_{r_{j}}$, then $m\left(\theta_{i}\right)=m\left(\theta_{j}\right)$, so the above construction is well defined. According to the sellers' incentive compatibility constraint for type $i$ to report $j$, one can write: $m\left(\theta_{i}\right) u_{i}\left(a_{i}\right)+s_{i} \geq$ $m\left(\theta_{j}\right) u_{i}\left(a_{j}\right)+s_{j}=m\left(\theta_{j}\right)\left(u_{i}\left(a_{j}\right)+\frac{s_{j}-t_{0}}{m\left(\theta_{j}\right)}\right)+t_{0}=m\left(\theta_{j}\right)\left(u_{i}\left(a_{i}\right)+\frac{s_{i}-t_{0}}{m\left(\theta_{i}\right)}\right)+t_{0}$ where the second equality follows from the assumption that $y_{r_{i}}=y_{r_{j}}$. This implies that $\left(m\left(\theta_{i}\right)-m\left(\theta_{j}\right)\right)\left(u_{i}\left(a_{i}\right)+\right.$ $\left.\frac{s_{i}-t_{0}}{m\left(\theta_{i}\right)}\right) \geq 0$. If $\theta_{k}>0$ for all $k$, then $t_{0}=0$ by construction, and due to the participation constraint for type $i, m\left(\theta_{i}\right) u_{i}\left(a_{i}\right)+s_{i} \geq 0$. If there exists $k$ such that $\theta_{k}=0$, then IC constraint for type $i$ to report $k$ implies that $m\left(\theta_{i}\right) u_{i}\left(a_{i}\right)+s_{i} \geq s_{k}=t_{0}$ for all $i$. Therefore, whether there exists $k$ with $\theta_{k}=0$ or not, $u_{i}\left(a_{i}\right)+\frac{s_{i}-t_{0}}{m\left(\theta_{i}\right)} \geq 0$. Thus, $m\left(\theta_{i}\right) \geq m\left(\theta_{j}\right)$. Similarly, by considering the sellers' incentive compatibility constraint for $j$ to report $i$, one can obtain $m\left(\theta_{j}\right) \geq m\left(\theta_{i}\right)$. Therefore, $m\left(\theta_{j}\right)=m\left(\theta_{i}\right)$.

Moreover, notice that $t_{0}$ is well defined, because if there are more than one $i$ with $\theta_{i}=0$, then $s_{i}$ must be the same for all of them, for otherwise, the sellers' incentive compatibility

\footnotetext{
${ }^{30}$ If there are more than two types with the same $y_{r_{i}}$, then keep the one with the lowest $\theta_{i}$ again, remove the rest, and construct $\gamma_{i}, \lambda\left(\left\{y_{r_{i}}\right\}\right)$ and $t\left(y_{r_{i}}\right)$ similarly.
} 
constraint in the definition of feasible mechanism is violated. Also, $y_{r_{i}}$ is well defined for all $i$, because $\theta_{i}$ cannot be equal to 0 , for all $i \in N$. Furthermore, $t(y)$ is well defined, since no $\theta_{i}$ can be equal to $\infty$. If $\theta_{i}$ goes to $\infty$ for some $i$, then the planner's budget-balance condition will be violated, because the planner needs to spend an infinite amount of resources to finance entry of buyers (the LHS of the planner's budget-balance condition goes to $-\infty$ ).

I show next that $\{\lambda, \mathcal{Y}, \Theta, \Gamma\}$ is implementable through $\left\{t, t_{0}\right\}$. Regarding sellers' optimal search, first consider $i \in N$. I need to show

$$
\begin{gathered}
U_{i} \equiv m\left(\theta_{r_{i}}\right)\left(u_{i}\left(a_{r_{i}}\right)+p_{r_{i}}\right)+t_{0} \geq m\left(\theta_{r_{j}}\right)\left(u_{i}\left(a_{r_{j}}\right)+p_{r_{j}}\right)+t_{0} \text { for } j \in N, \\
m\left(\theta_{r_{i}}\right)\left(u_{i}\left(a_{r_{i}}\right)+p_{r_{i}}\right) \geq 0 .
\end{gathered}
$$

To show the above inequalities, note that

$$
m\left(\theta_{r_{i}}\right)\left(u_{i}\left(a_{r_{i}}\right)+p_{r_{i}}\right)=m\left(\theta_{i}\right) u_{i}\left(a_{i}\right)+s_{i}-t_{0} \geq m\left(\theta_{i}\right) u_{i}\left(a_{j}\right)+s_{j}-t_{0} \text { for all } j,
$$

where the equality follows from the construction of $y_{r_{i}}$ and the inequality follows from the incentive compatibility condition in the direct mechanism. The RHS equals to 0 if $j \notin N$ and equals to $m\left(\theta_{r_{j}}\right)\left(u_{i}\left(a_{r_{j}}\right)+p_{r_{j}}\right)$ if $j \in N$. Thus, (15) is established. Regarding (16), if $N \neq\{1,2, \ldots, I\}$, then consider a $j \in\{1,2, \ldots, I\} \backslash N$. Since $\theta_{j}=0$ by definition of $N$, then IC in the feasible mechanism implies (16). If $N=\{1,2, \ldots, I\}$, then $t_{0}=0$ and $m\left(\theta_{r_{i}}\right)\left(u_{i}\left(a_{r_{i}}\right)+p_{r_{i}}\right) \geq 0$ due to the participation constraint in the direct mechanism.

To show $U_{i} \geq m(\Theta(y))\left(u_{i}(a)+p\right)+t_{0}$ for all $y \in \mathcal{Y}$ and $i \notin N$, I need to show $t_{0} \geq$ $m\left(\theta_{r_{j}}\right)\left(u_{i}\left(a_{r_{j}}\right)+p_{r_{j}}\right)+t_{0}$ for all $j \in N$, but

$m\left(\theta_{r_{j}}\right)\left(u_{i}\left(a_{r_{j}}\right)+p_{r_{j}}\right)+t_{0}=m\left(\theta_{j}\right)\left(u_{i}\left(a_{j}\right)+p_{j}\right)+s_{j} \leq m\left(\theta_{i}\right) u_{i}\left(a_{i}\right)+s_{i}=s_{i}=t_{0}$ for all $j \in N$.

The first equality follows from the construction of $y_{r_{j}}$ and $j \in N$. The inequality follows from the incentive compatibility condition in the direct mechanism. The next equality holds because $\theta_{i}=0$ as $i \notin N$. The last equality holds due to the construction of $t_{0}$ and that $i \notin N$.

I now show formally that condition (ii) is satisfied. By construction of $\Theta($.$) and \Gamma($.$) and$ as shown above: $U_{i} \geq m(\Theta(y))\left(u_{i}(a)+p\right)+t_{0}$ for all $y \in \mathcal{Y}$ with equality if $\Theta(y)<\infty$ and $\gamma_{i}(y)>0$. The inequality also holds for $y \notin \mathcal{Y}$ due to the construction of $\Theta($.$) and \Gamma($.$) .$ Given $y$, if $u_{i}(a)+p<0$ for some $i$, then $i \notin K(y)$. Thus, if $K(y)=\emptyset$, then $\Theta(y)=\infty$. If $K(y) \neq \emptyset$, then $\gamma_{n_{o}}(y)=1$ for some $n_{o} \in K(y)$, therefore $\gamma_{i}(y)=0$.

Buyers' profit maximization and free-entry condition holds because of the following reasons. For $y \in \mathcal{Y}$, there exists $i \in N$ such that $y=y_{r_{i}}$. Thus, $q\left(\theta_{r_{i}}\right)\left(v_{i}\left(a_{r_{i}}\right)-p_{r_{i}}-t\left(y_{r_{i}}\right)\right)=$ $k$ by construction of $t($.$) , so this condition holds with equality for y \in \mathcal{Y}$. For $y \notin \mathcal{Y}$, $q(\Theta(y)) \sum \gamma_{i}(y)\left(v_{i}(a)-p-t(y)\right)<k$ because of the construction of $t(y)$. Therefore, condition (i) is satisfied. ${ }^{31}$

\footnotetext{
${ }^{31}$ It is clear from this step of the proof that the restrictions on off-the-equilibrium-path beliefs do not play any role in my analysis. That is, any other off-the-equilibrium-path beliefs would work with the proposed taxes. This is because the planner does not face any restriction on the tax amount that he can impose.

Moreover, one may want to add another restriction that buyers' payoff from trade before taxation must be positive. Otherwise, buyers may want to fake trading. Adding this restriction may be interesting in some contexts, but it is beyond the scope of this paper.
} 
Feasibility condition is obviously satisfied following the construction of $\lambda$.

Planner's budget-balance condition is satisfied because:

$$
\begin{gathered}
\int q(\Theta) t(y) d \lambda(\{y\})-t_{0} \geq \sum_{i \in N} q\left(\theta_{i}\right)\left(v_{i}\left(a_{i}\right)-p_{i}-\frac{s_{i}-t_{0}}{m\left(\theta_{i}\right)}-\frac{k}{q\left(\theta_{i}\right)}\right) \pi_{i} \theta_{i}-t_{0} \\
=\sum_{i \in N} \pi_{i}\left[m\left(\theta_{i}\right) v_{i}\left(a_{i}\right)-s_{i}-k \theta_{i}\right]+\sum_{i \notin N} \pi_{i}\left[m\left(\theta_{i}\right) v_{i}\left(a_{i}\right)-s_{i}-k \theta_{i}\right] \geq 0 .
\end{gathered}
$$

The first inequality holds due to the construction of the allocation. Note that if several types have the same $y_{r_{i}}$, then it was shown that $m\left(\theta_{r_{i}}\right)=m\left(\theta_{r_{j}}\right)$ and if $\theta_{r_{i}} \neq \theta_{r_{j}}$, then the lowest one was chosen. The equality holds due to the fact that for $i \notin N, \theta_{i}=0$ and $s_{i}=t_{0}$. The last inequality holds due to the budget-balance condition in the feasible mechanism.

Proof of Theorem 1. As stated in the text, the budget-balance constraint should be binding in the planner's problem, so the problem can be written as:

$$
\max _{\left\{\theta_{i}, a_{i}, s_{i}\right\}} \sum_{i=1}^{I} \pi_{i}\left(m\left(\theta_{i}\right)\left(u_{i}\left(a_{i}\right)+v_{i}\left(a_{i}\right)\right)-k \theta_{i}\right)
$$

subject to $\mathrm{IC}, \mathrm{PC}$ and $\mathrm{BB}$ with equality.

\section{Step 1: Constructing an allocation close to the equilibrium allocation.}

I begin from the equilibrium allocation and modify it in a particular way to improve welfare. Consider type $r$ such that $0<U_{r}<U_{r}^{F B}$. Such a type exists because the equilibrium fails to achieve the first best. I use the same set of problems proposed by GSW to construct a feasible mechanism and show that it yields strictly higher welfare for the planner than the equilibrium allocation. According to Propositions 1-3 in their paper, GSW show that the following set of problems characterizes the equilibrium.

Problem $1\left(P_{i}\right)$.

$$
\begin{gathered}
\max _{\theta \in[0, \infty],(a, p) \in \bar{Y}}\left\{m(\theta)\left(u_{i}(a)+p\right)\right\} \\
\text { subject to: } q(\theta)\left(v_{i}(a)-p\right) \geq k, \\
m(\theta)\left(u_{j}(a)+p\right) \leq \bar{U}_{j} \text { for all } j<i .
\end{gathered}
$$

More precisely, define problem $P$ to be the set of problems $P_{i}$ for all $i$. Denote by $I^{*} \subseteq$ $\{1,2, \ldots, I\}$ the set of types such that the constraint set in $P_{i}$ is non-empty and $\bar{U}_{i}$ is strictly greater than 0 . Let $\bar{U}_{i}$ be the value of the objective function in $P_{i}$ given $\left(\bar{U}_{1}, \bar{U}_{2}, \ldots, \bar{U}_{i-1}\right)$ if $i \in I^{*}$, and $\bar{U}_{i}=0$ otherwise. For any $i \in I^{*}$, let $\left(\bar{\theta}_{i}, \bar{a}_{i}, \bar{p}_{i}\right)$ denote the solution to $P_{i}$ given $\left(\bar{U}_{1}, \bar{U}_{2}, \ldots, \bar{U}_{i-1}\right)$. Now, consider the following problem for type $r$, which is basically a perturbation of the above problem in a specific way. 
Problem $2\left(P_{r}(\epsilon), \epsilon \geq 0\right)$.

$$
\begin{gathered}
\max _{\theta \in[0, \infty],(a, p) \in \bar{Y}}\left\{m(\theta)\left(u_{r}(a)+p\right)\right\} \\
\text { subject to: } q(\theta)\left(v_{r}(a)-p\right) \geq k, \\
m(\theta)\left(u_{j}(a)+p\right) \leq \bar{U}_{j}+\epsilon \text { for all } j<r .
\end{gathered}
$$

Let $\bar{U}_{r}(\epsilon)$ be the value of the objective function and let $\left(\bar{\theta}_{r}(\epsilon), \bar{a}_{r}(\epsilon), \bar{p}_{r}(\epsilon)\right)$ denote the solution to this problem given $\left(\bar{U}_{1}(\epsilon), \bar{U}_{2}(\epsilon), \ldots, \bar{U}_{r-1}(\epsilon)\right)$. All $\bar{U}_{r}(\epsilon)$ and $\left(\bar{\theta}_{r}(\epsilon), \bar{a}_{r}(\epsilon), \bar{p}_{r}(\epsilon)\right)$ are functions (correspondences in general) of $\epsilon$, and at $\epsilon=0$, they are equal to their equilibrium counterparts.

\section{Step 2: Eliminating $p$ from $P_{r}(\epsilon)$ by showing that its first constraint is binding.}

Assume by way of contradiction that the constraint is not binding. First note that $\bar{\theta}_{r}(\epsilon)>0$ because $\bar{U}_{r}(0)>0$ and that the constraint set for $\epsilon>0$ includes that of $\epsilon=0$. By Assumption 1(ii), for every $\tau>0$, there exists an $a^{\prime} \in B_{\tau}\left(\bar{a}_{r}(\epsilon)\right)$ such that $u_{r}\left(a^{\prime}\right)>u_{r}\left(\bar{a}_{r}(\epsilon)\right)$ and $u_{j}\left(a^{\prime}\right)<u_{j}\left(\bar{a}_{r}(\epsilon)\right)$ for all $j<r$.

Set $\tau>0$ sufficiently small such that $q\left(\bar{\theta}_{r}(\epsilon)\right)\left(v_{r}\left(a^{\prime}\right)-\bar{p}_{r}(\epsilon)\right) \geq k$ for all $B_{\tau}\left(\bar{a}_{r}(\epsilon)\right)$. Now consider $\left(\bar{\theta}_{r}(\epsilon), a^{\prime}, \bar{p}_{r}(\epsilon)\right)$. The first constraint in problem $P_{r}(\epsilon)$ is satisfied following the choice of $\tau$. Other constraints are satisfied because: $m\left(\bar{\theta}_{r}(\epsilon)\right)\left(u_{j}\left(a^{\prime}\right)+\bar{p}_{r}(\epsilon)\right)<$ $m\left(\bar{\theta}_{r}(\epsilon)\right)\left(u_{j}\left(\bar{a}_{r}(\epsilon)\right)+\bar{p}_{r}(\epsilon)\right) \leq \bar{U}_{j}+\epsilon$ for all $j<r$. But the value of the objective function is now higher: $m\left(\bar{\theta}_{r}(\epsilon)\right)\left(u_{r}\left(a^{\prime}\right)+\bar{p}_{r}(\epsilon)\right)>m\left(\bar{\theta}_{r}(\epsilon)\right)\left(u_{r}\left(\bar{a}_{r}(\epsilon)\right)+\bar{p}_{r}(\epsilon)\right)$. This is a contradiction with $\left(\bar{\theta}_{r}(\epsilon), \bar{a}_{r}(\epsilon), \bar{p}_{r}(\epsilon)\right)$ being a solution to problem $P_{r}(\epsilon)$.

Given that the first constraint is always binding, $P_{r}(\epsilon)$ can be written as follows after eliminating $p$ :

$$
\begin{gathered}
\max _{\theta \in[0, \infty],(., a) \in \bar{Y}}\left\{m(\theta)\left(u_{r}(a)+v_{r}(a)\right)-k \theta\right\} \\
\text { subject to } m(\theta)\left(u_{j}(a)+v_{r}(a)\right)-k \theta \leq \bar{U}_{j}+\epsilon \text { for all } j<r .
\end{gathered}
$$

This is the problem that was used in the text. Since type $r$ does not achieve the first best, some constraints of $P_{r}(0)$ must be binding in equilibrium. Following Assumption 1(iii), $m(\theta)\left(u_{i}(a)+v_{i}(a)\right)-k \theta$ has a single peak for all $i$, so locally relaxing the constraints of the problem by increasing $\epsilon=0$ to a strictly positive number increases $\bar{U}_{r}$, which in turn improves welfare.

Since continuity in $\epsilon$ is needed later, in the next step I apply the theorem of the maximum to this problem and show that $\bar{U}_{r}(\epsilon)$ is continuous in $\epsilon$ and $\bar{a}_{r}(\epsilon), \bar{\theta}_{r}(\epsilon)$ and $\bar{p}_{r}(\epsilon)$ are all upperhemi-continuous (UHC) in $\epsilon$.

\section{Step 3: Continuity in $\epsilon$.}

Claim 1. There exists $\epsilon_{0}>0$ such that $\bar{U}_{r}(\epsilon)$ is continuous and $\bar{a}_{r}(\epsilon), \bar{\theta}_{r}(\epsilon)$ and $\bar{p}_{r}(\epsilon)$ are UHC for $\epsilon \in\left[0, \epsilon_{0}\right]$.

Claim 2. There exists $\epsilon_{j}>0$ for any $j>r$ such that $e_{j}(\epsilon)>0$ for $\epsilon \in\left[0, \epsilon_{j}\right]$ where

$$
e_{j}(\epsilon) \equiv \bar{U}_{j}-m\left(\bar{\theta}_{r}(\epsilon)\right)\left(u_{j}\left(\bar{a}_{r}(\epsilon)\right)+p_{r}(\epsilon)\right) \text { for all } j>r .
$$


Intuitively, $e_{j}(\epsilon)$ is the net loss of type $j$ by reporting type $r$. It is shown here that it is strictly positive.

Proof of claim 1: First, the constraint set is compact because $a \in \bar{A}$ and $\bar{A}$ is compact. Also, $\theta$ lies in a closed interval that is a subset of $\mathbb{R}_{+}$. Suppose by way of contradiction that $\theta$ is unbounded, then the objective function goes to $-\infty$, because $m(\theta) \leq 1, u_{i}(a)+v_{i}(a)$ is bounded, and $k>0$. Therefore, it can be assumed without loss of generality that $(\theta, a) \in$ $[0, M] \times \bar{A}$ for some $M \in \mathbb{R}_{+}$. Second, it is easy to verify that the constraint correspondence is continuous in $\epsilon$ following the continuity of $m, u_{i}$ and $v_{i}$. Third, the objective function is continuous in $(\theta, a)$ and $\epsilon$. All requirements of the theorem of the maximum holds, so $\bar{U}_{r}(\epsilon)$ is continuous in $\epsilon$ and $\bar{a}_{r}(\epsilon), \bar{\theta}_{r}(\epsilon)$ and $\bar{p}_{r}(\epsilon)$ are all UHC in $\epsilon$. Both $\bar{\theta}_{r}(\epsilon)$ and $\bar{a}_{r}(\epsilon)$ are UHC in $\epsilon$, and $m($.$) and u_{i}($.$) are continuous functions, therefore, m\left(\bar{\theta}_{r}(\epsilon)\right)$ and $u_{i}\left(\bar{a}_{r}(\epsilon)\right)$ are UHC in $\epsilon$. (See Theorem 17.23 in Aliprantis and Border (1986).)

Proof of claim 2: Since $e_{j}(\epsilon)$ is just the sum of UHC correspondences, $e_{j}(\epsilon)$ is also UHC. But $\left.e_{j}(\epsilon)\right|_{\epsilon=0}>0$ according to Lemma 3. I show below that because $e_{j}(\epsilon)$ is UHC in an interval close to 0 and its value at 0 is strictly positive, there must exist a neighborhood $\left[0, \epsilon_{j}\right]$ for some $\epsilon_{j}>0$ such that $e_{j}(\epsilon)$ is strictly positive, too. Suppose by way of contradiction that such a neighborhood does not exist. This implies that there exists $j>r$ such that for any $\epsilon>0$, there exists a function $e(\epsilon) \in e_{j}(\epsilon)$ with $e(\epsilon) \leq 0$. Consider $\left\{\epsilon_{s}\right\}_{s \in \mathbb{N}}$ where $\epsilon_{s}=\frac{1}{s}$. Since $e_{j}($.$) is UHC and because \epsilon_{s} \rightarrow 0$, there exists a convergent sub-sequence $\left\{e_{s}\right\}_{s \in \mathbb{N}}$ of $\left\{e\left(\epsilon_{s}\right)\right\}_{s \in \mathbb{N}}$ such that its limit point is in $e_{j}(0)$. This is a contradiction, because $e_{j}(0)>0$ and $e_{s} \leq 0$ for all $s$, so its limit point cannot be a strictly positive number.

\section{Step 4: Proposing a direct mechanism.}

I propose the following direct mechanism, $\left\{\left(\theta_{i}, a_{i}, s_{i}\right)\right\}_{i \in\{1,2, \ldots, I\}}$, such that

$$
\left(\theta_{i}, a_{i}, s_{i}\right)= \begin{cases}\left(\bar{\theta}_{i}, \bar{a}_{i}, m\left(\bar{\theta}_{i}\right) \bar{p}_{i}+\epsilon-\tilde{\epsilon}\right) & \text { if } 1 \leq i<r \text { or } i \notin I^{*} \\ \left(\bar{\theta}_{i} \bar{a}_{i}, m\left(\bar{\theta}_{i}\right) \bar{p}_{i}-\tilde{\epsilon}\right) & \text { if } i \in\{r+1, r+2, \ldots, I\} \cap I^{*} \\ \left(\bar{\theta}_{i}(\epsilon) \bar{a}_{i}(\epsilon), m\left(\bar{\theta}_{i}(\epsilon)\right) \bar{p}_{i}(\epsilon)-\tilde{\epsilon}\right) & \text { if } i=r\end{cases}
$$

where $\tilde{\epsilon} \equiv\left(\sum_{i=1}^{r-1} \pi_{i}+\sum_{i=r+1, i \notin I^{*}}^{I} \pi_{i}\right) \epsilon$. Note that for $i \notin I^{*}, \bar{\theta}_{i}=0$, and $\bar{a}_{i}$ and $\bar{s}_{i}$ are chosen arbitrarily.

I show below that if $\epsilon>0$ is sufficiently close to 0 , then this mechanism is feasible and yields strictly higher welfare than the equilibrium.

\section{Step 5: The proposed mechanism is incentive compatible.}

Due to my construction method, it is rather obvious that all IC constraints hold, because all downward IC constraints are slack in the original allocation and the new allocation was constructed in the neighborhood of that, while keeping all upward IC constraints satisfied. Now I formally prove that IC constraints hold for all possible cases and show that no seller of type $i$ gains by reporting type $j$ for sufficiently small $\epsilon>0$ for all $i, j$.

Case 1: $i \notin I^{*}$ or $i<r ; j \notin I^{*}$ or $j<r$

$m\left(\theta_{i}\right) u_{i}\left(a_{i}\right)+s_{i}=m\left(\bar{\theta}_{i}\right)\left(u_{i}\left(\bar{a}_{i}\right)+\bar{p}_{i}\right)+\epsilon-\tilde{\epsilon} \geq m\left(\bar{\theta}_{j}\right)\left(u_{i}\left(\bar{a}_{j}\right)+\bar{p}_{j}\right)+\epsilon-\tilde{\epsilon}=m\left(\theta_{j}\right) u_{i}\left(a_{j}\right)+s_{j}$, 
where the inequality holds following the sellers' optimal search in the equilibrium allocation.

Case 2: $i \notin I^{*}$ or $i<r ; j=r$

$m\left(\theta_{i}\right) u_{i}\left(a_{i}\right)+s_{i}=m\left(\bar{\theta}_{i}\right)\left(u_{i}\left(\bar{a}_{i}\right)+\bar{p}_{i}\right)+\epsilon-\tilde{\epsilon} \geq m\left(\bar{\theta}_{j}(\epsilon)\right)\left(u_{i}\left(\bar{a}_{j}(\epsilon)\right)+\bar{p}_{j}(\epsilon)\right)-\tilde{\epsilon}=m\left(\theta_{j}\right) u_{i}\left(a_{j}\right)+s_{j}$,

where the inequality is basically the same as one of the constraints in problem $P_{r}(\epsilon)$ if $i \in I^{*}$. If $i \notin I^{*}$, then the inequality holds because $0 \geq m\left(\bar{\theta}_{j}\right)\left(u_{i}\left(\bar{a}_{j}\right)+\bar{p}_{j}\right)$.

Case 3: $i \notin I^{*}$ or $i<r ; j \in I^{*}$ and $j>r$

$$
m\left(\theta_{i}\right) u_{i}\left(a_{i}\right)+s_{i}=m\left(\bar{\theta}_{i}\right)\left(u_{i}\left(\bar{a}_{i}\right)+\bar{p}_{i}\right)+\epsilon-\tilde{\epsilon} \geq m\left(\bar{\theta}_{j}\right)\left(u_{i}\left(\bar{a}_{j}\right)+\bar{p}_{j}\right)-\tilde{\epsilon}=m\left(\theta_{j}\right) u_{i}\left(a_{j}\right)+s_{j}
$$

where, again, the inequality holds because in the equilibrium allocation: $m\left(\bar{\theta}_{i}\right)\left(u_{i}\left(\bar{a}_{i}\right)+\bar{p}_{i}\right) \geq$ $m\left(\bar{\theta}_{j}\right)\left(u_{i}\left(\bar{a}_{j}\right)+\bar{p}_{j}\right)$ if $i \in I^{*}$, and $0 \geq m\left(\bar{\theta}_{j}\right)\left(u_{i}\left(\bar{a}_{j}\right)+\bar{p}_{j}\right)$ if $i \notin I^{*}$.

Case 4: $i=r ; j \notin I^{*}$ or $j<r$

$$
\begin{aligned}
m\left(\theta_{i}\right) u_{i}\left(a_{i}\right)+s_{i} & =m\left(\bar{\theta}_{i}(\epsilon)\right)\left(u_{i}\left(\bar{a}_{i}(\epsilon)\right)+\bar{p}_{i}(\epsilon)\right)-\tilde{\epsilon}>m\left(\bar{\theta}_{i}(0)\right)\left(u_{i}\left(\bar{a}_{i}(0)\right)+\bar{p}_{i}(0)\right)-\tilde{\epsilon} \\
& \geq m\left(\bar{\theta}_{j}\right)\left(u_{i}\left(\bar{a}_{j}\right)+\bar{p}_{j}\right)+\epsilon-\tilde{\epsilon}=m\left(\theta_{j}\right) u_{i}\left(a_{j}\right)+s_{j} .
\end{aligned}
$$

The first inequality follows from the fact that by making strictly positive transfers to types below $r$, type $r$ receives a strictly higher payoff due to the relaxation of the constraints of $P_{r}$. The second inequality follows from Lemma 3 stating that higher types are strictly worse off by reporting lower types in the equilibrium, i.e., when $\epsilon=0$. Therefore, the inequality holds for $\epsilon>0$ sufficiently small. For $j \notin I^{*}$, the inequality follows from the fact that $U_{i}^{E Q}>0=U_{j}^{E Q}$ and $\epsilon$ is sufficiently small.

Case 5: $i=r ; j \in I^{*}$ and $j>r$

$$
\begin{aligned}
m\left(\theta_{i}\right) u_{i}\left(a_{i}\right)+s_{i}= & m\left(\bar{\theta}_{i}(\epsilon)\right)\left(u_{i}\left(\bar{a}_{i}(\epsilon)\right)+\bar{p}_{i}(\epsilon)\right)-\tilde{\epsilon}>m\left(\bar{\theta}_{i}(0)\right)\left(u_{i}\left(\bar{a}_{i}(0)\right)+\bar{p}_{i}(0)\right)-\tilde{\epsilon} \\
& \geq m\left(\bar{\theta}_{j}\right)\left(u_{i}\left(\bar{a}_{j}\right)+\bar{p}_{j}\right)-\tilde{\epsilon}=m\left(\theta_{j}\right) u_{i}\left(a_{j}\right)+s_{j} .
\end{aligned}
$$

The first inequality is the same as the first one in the previous case. The second inequality is the same as one of the constraints in $P_{j}$.

Case 6: $i \in I^{*}$ and $i>r ; j \notin I^{*}$ or $j<r$

$$
m\left(\theta_{i}\right) u_{i}\left(a_{i}\right)+s_{i}=m\left(\bar{\theta}_{i}\right)\left(u_{i}\left(\bar{a}_{i}\right)+\bar{p}_{i}\right)-\tilde{\epsilon} \geq m\left(\bar{\theta}_{j}\right)\left(u_{i}\left(\bar{a}_{j}\right)+\bar{p}_{j}\right)+\epsilon-\tilde{\epsilon}=m\left(\theta_{j}\right) u_{i}\left(a_{j}\right)+s_{j},
$$

where the inequality holds for the same reason as in the second inequality in case 4 .

Case 7: $i \in I^{*}$ and $i>r ; j=r$

$m\left(\theta_{i}\right) u_{i}\left(a_{i}\right)+s_{i}=m\left(\bar{\theta}_{i}\right)\left(u_{i}\left(\bar{a}_{i}\right)+\bar{p}_{i}\right)-\tilde{\epsilon} \geq m\left(\bar{\theta}_{j}(\epsilon)\right)\left(u_{i}\left(\bar{a}_{j}(\epsilon)\right)+\bar{p}_{j}(\epsilon)\right)-\tilde{\epsilon}=m\left(\theta_{j}\right) u_{i}\left(a_{j}\right)+s_{j}$,

where part 2 of claim 2 is used here to establish the inequality for sufficiently small $\epsilon$.

Case 8: $i \in I^{*}$ and $i>r ; j \in I^{*}$ and $j>r$

$$
m\left(\theta_{i}\right) u_{i}\left(a_{i}\right)+s_{i}=m\left(\bar{\theta}_{i}\right)\left(u_{i}\left(\bar{a}_{i}\right)+\bar{p}_{i}\right)-\tilde{\epsilon} \geq m\left(\bar{\theta}_{j}\right)\left(u_{i}\left(\bar{a}_{j}\right)+\bar{p}_{j}\right)-\tilde{\epsilon}=m\left(\theta_{j}\right) u_{i}\left(a_{j}\right)+s_{j},
$$


where the inequality holds following from the sellers' optimal search in the equilibrium allocation.

\section{Step 6: The proposed mechanism satisfies $\mathrm{PC}$ and BB with equality.}

The budget-balance condition is obviously satisfied with equality due to the construction of $\tilde{\epsilon}$. Regarding PC, consider the following cases. If $i \notin I^{*}$ or $i<r$, then type $i$ is better off by the amount of $\epsilon-\tilde{\epsilon}>0$. If $i=r$, then $m\left(\theta_{i}\right) u_{i}\left(a_{i}\right)+s_{i}=m\left(\bar{\theta}_{i}(\epsilon)\right)\left(u_{i}\left(\bar{a}_{i}(\epsilon)\right)+\bar{p}_{i}(\epsilon)\right)-\tilde{\epsilon}>$ $m\left(\bar{\theta}_{i}(0)\right)\left(u_{i}\left(\bar{a}_{i}(0)\right)+\bar{p}_{i}(0)\right)-\tilde{\epsilon}>0$ where the last inequality holds following the facts that type $r$ gets a strictly positive payoff in equilibrium and $\epsilon>0$ is small. If $i \in I^{*}$ and $i>r$, $m\left(\theta_{i}\right) u_{i}\left(a_{i}\right)+s_{i}=m\left(\bar{\theta}_{i}\right)\left(u_{i}\left(\bar{a}_{i}\right)+\bar{p}_{i}\right)-\tilde{\epsilon}>0$ where the inequality holds true following the same reasons as in the previous case.

\section{Step 7: The proposed mechanism achieves higher welfare than equilibrium.}

The surplus created by types $i \in I \backslash\{r\}$ is the same as that in equilibrium but type $r$ has created strictly more surplus, so the total amount of surplus has increased.

Lemma 3. There exist $I^{*} \subseteq\{1,2, . ., I\},\left\{\bar{U}_{i}\right\}_{i \in\{1,2, . ., I\}}$ and $\left\{\left(\bar{\theta}_{i}, \bar{a}_{i}, \bar{p}_{i}\right)\right\}_{i \in I^{*}}$ that solve problem $P$. The following holds at any solution to $P_{i}$ for $i \in I^{*}$ :

$$
\begin{gathered}
q\left(\bar{\theta}_{i}\right)\left(v_{i}\left(\bar{a}_{i}\right)-\bar{p}_{i}\right)=k, \\
m\left(\bar{\theta}_{i}\right)\left(u_{j}\left(\bar{a}_{i}\right)+\bar{p}_{i}\right)<\bar{U}_{j} \text { for all } j>i .
\end{gathered}
$$

Proof. For the existence proof and also the fact that the first constraint in $P_{i}$ is binding, see Lemma 1 in GSW. Here, I prove only the last part, stating that higher types are strictly worse off if they apply to submarkets designed for lower types. GSW show only that IC constraints are satisfied, but they do not show whether they are binding or not. I show that downward IC constraints are satisfied and never binding following the assumption that $v_{i}(a)$ is strictly increasing in $i$ for all $a \in \bar{A}$, while they simply assume weak monotonicity.

Fix $i \in I^{*}$. Assume by way of contradiction that there exists $r$ such that $r>i$ and $m\left(\bar{\theta}_{i}\right)\left(u_{r}\left(\bar{a}_{i}\right)+\bar{p}_{i}\right) \geq \bar{U}_{r}$. Denote the smallest such $r$ by $h$. That is, $m\left(\bar{\theta}_{i}\right)\left(u_{j}\left(\bar{a}_{i}\right)+\bar{p}_{i}\right)<$ $\bar{U}_{j}$ for all $i \leq j<h$, and $m\left(\bar{\theta}_{i}\right)\left(u_{h}\left(\bar{a}_{i}\right)+\bar{p}_{i}\right) \geq \bar{U}_{h}$. Now I show that $\left(\bar{\theta}_{i}, \bar{a}_{i}, \bar{p}_{i}\right)$ is feasible for $P_{h}$. The first constraint in $P_{h}$ is satisfied because $q\left(\bar{\theta}_{i}\right)\left(v_{h}\left(\bar{a}_{i}\right)-\bar{p}_{i}\right)>q\left(\bar{\theta}_{i}\right)\left(v_{i}\left(\bar{a}_{i}\right)-\bar{p}_{i}\right) \geq k$, where the first inequality follows from the fact that $q\left(\bar{\theta}_{i}\right)>0$ as $i \in I^{*}$ and from Assumption 1(i) as $a_{i} \in \bar{A}$. The latter holds true for the following reason: $\left(\bar{\theta}_{i}, \bar{a}_{i}, \bar{p}_{i}\right)$ is feasible for $P_{i}$, so $q\left(\bar{\theta}_{i}\right)\left(v_{i}\left(\bar{a}_{i}\right)-\bar{p}_{i}\right) \geq k$ and $\bar{\theta}_{i} \geq 0$, thus $q(0)\left(v_{i}\left(\bar{a}_{i}\right)-\bar{p}_{i}\right) \geq k$. Also $m\left(\bar{\theta}_{i}\right)\left(u_{i}\left(\bar{a}_{i}\right)+\bar{p}_{i}\right)=\bar{U}_{i}$, but $\bar{U}_{i}>0$ by construction of $I^{*}$, therefore, $u_{i}\left(\bar{a}_{i}\right)+\bar{p}_{i} \geq 0$, thus $\bar{a}_{i} \in \bar{A}$. Regarding other constraints, $m\left(\bar{\theta}_{i}\right)\left(u_{j}\left(\bar{a}_{i}\right)+\bar{p}_{i}\right) \leq \bar{U}_{j}$ holds true for any $j$ with $i<j<h$ by construction of $h$, and holds true for any $j$ with $j \leq i$, because $\left(\bar{\theta}_{i}, \bar{a}_{i}, \bar{p}_{i}\right)$ is feasible for $P_{i}$.

Choose $\left(a^{\prime}, p^{\prime}\right) \in \bar{A} \times \mathbb{R}$ sufficiently close to $\left(\bar{a}_{i}, \bar{p}_{i}\right)$ such that

$$
\max \left\{\max _{j<h}\left\{u_{j}\left(a^{\prime}\right)-u_{j}\left(\bar{a}_{i}\right)\right\}, \bar{p}_{i}-v_{h}\left(a^{\prime}\right)+\frac{k}{q\left(\bar{\theta}_{i}\right)}\right\}<\bar{p}_{i}-p^{\prime}<u_{h}\left(a^{\prime}\right)-u_{h}\left(\bar{a}_{i}\right) .
$$


Such $\left(a^{\prime}, p^{\prime}\right)$ exists because the LHS is strictly less than the RHS for $a^{\prime}$ sufficiently close to $\bar{a}_{i}$ owing to Assumption 1(ii) and because $q\left(\bar{\theta}_{i}\right)\left(v_{h}\left(\bar{a}_{i}\right)-\bar{p}_{i}\right)>k$. The above inequalities can be written in the following simpler form:

$$
\begin{gathered}
u_{h}\left(a^{\prime}\right)+p^{\prime}>u_{h}\left(\bar{a}_{i}\right)+\bar{p}_{i}, \\
u_{j}\left(a^{\prime}\right)+p^{\prime}<u_{j}\left(\bar{a}_{i}\right)+\bar{p}_{i} \text { for all } j<h, \\
\text { and } q\left(\bar{\theta}_{i}\right)\left(v_{h}\left(a^{\prime}\right)-\bar{p}_{i}\right) \geq k .
\end{gathered}
$$

The claim is that $\left(\bar{\theta}_{i}, a^{\prime}, p^{\prime}\right)$ is feasible for $P_{h}$ but delivers a strictly higher utility for type $h$. The first constraint in $P_{h}$ is satisfied by the choice of $a^{\prime}$. All incentive compatibility constraints are satisfied because $m\left(\bar{\theta}_{i}\right)\left(u_{j}\left(a^{\prime}\right)+p^{\prime}\right)<m\left(\bar{\theta}_{i}\right)\left(u_{j}\left(\bar{a}_{i}\right)+\bar{p}_{i}\right) \leq \bar{U}_{j}$ for all $j<h$, where the weak inequality follows from the fact that $\left(\bar{\theta}_{i}, \bar{a}_{i}, \bar{p}_{i}\right)$ is feasible for $P_{h}$. The value of the objective function is strictly greater than $\bar{U}_{h}$ because $m\left(\bar{\theta}_{i}\right)\left(u_{h}\left(a^{\prime}\right)+p^{\prime}\right)>m\left(\bar{\theta}_{i}\right)\left(u_{h}\left(\bar{a}_{i}\right)+\right.$ $\left.\bar{p}_{i}\right) \geq \bar{U}_{h} \geq 0$. Therefore, the constraint set of $P_{h}$ is non-empty and $\left(\bar{\theta}_{i}, a^{\prime}, p^{\prime}\right)$ delivers a strictly positive value for the objective function. Thus $h \in I^{*}$, but it was just shown that $\bar{U}_{h}$ is not the maximized value for $P_{h}$. This is a contradiction.

Proof of Theorem 2. Suppose such $r$ exists. I propose the following direct mechanism, which implements an allocation in the neighborhood of the equilibrium allocation:

$$
\left(\theta_{i}, a_{i}, s_{i}\right)= \begin{cases}\left(\bar{\theta}_{i}, \bar{a}_{i}, m\left(\bar{\theta}_{i}\right) \bar{p}_{i}+\epsilon-\tilde{\epsilon}\right) & \text { if } 1 \leq i<r \\ \left(\bar{\theta}_{i}, \bar{a}_{i}, m\left(\bar{\theta}_{i}\right) \bar{p}_{i}\right) & \text { if } r<i \leq I, \\ \left(\bar{\theta}_{i}(\epsilon), \bar{a}_{i}(\epsilon), m\left(\bar{\theta}_{i}(\epsilon)\right) \bar{p}_{i}(\epsilon)-\tilde{\epsilon}\right) & \text { if } i=r\end{cases}
$$

where $\tilde{\epsilon} \equiv \frac{\sum_{j=1}^{r-1} \pi_{j}}{\sum_{j=1}^{r} \pi_{j}} \epsilon$. First, types $1,2, \ldots, r-1$ are given $\epsilon$ units of the numeraire good. Next, the problem for type $r$ is solved again to characterize new $\theta, a$ and $p$ to be allocated to type $r$ and then types $1,2, \ldots, r$ are taxed in the lump sum way by amount $\tilde{\epsilon}$. Note that in the proof of Theorem 1, to finance the transfers to types $1,2, \ldots, r-1$, the planner taxed all types, but here, types above $r$ are not taxed as we do not want them to become worse off relative to the equilibrium allocation, because Pareto efficiency is concerned here. ${ }^{32}$ Finally, it is shown that this mechanism is feasible and all types are weakly better off and some types are strictly better off than equilibrium.

As shown in Lemma 3, the free-entry condition is always binding, so the problem for type $r$ can be written as

$$
\begin{gathered}
\max _{\theta \in[0, \infty],(., a) \in \bar{Y}}\left\{m(\theta)\left(u_{r}(a)+v_{r}(a)\right)-k \theta\right\} \\
\text { subject to } m(\theta)\left(u_{j}(a)+v_{r}(a)\right)-k \theta \leq \bar{U}_{j}+\epsilon \text { for all } j<r,
\end{gathered}
$$

\footnotetext{
${ }^{32}$ There are positive gains from trade for all types, so all types are active in equilibrium. I use this fact in the proof to ensure that when making $\epsilon$ transfers to types lower than $r$, there is no need to make transfers to the types above $r$ who are inactive. Even if this assumption fails to hold, as long as all types with positive gains from trade are active in equilibrium, the same logic as in the proof of Theorem 1 will go through, but types higher than $r$ with no gains from trade should be treated similarly to those types below $r$. To simplify the exposition and save space, I focus on this simpler case where $U_{i}^{F B}>0$ for all $i$ in this proof.
} 
where $\epsilon$ is the amount of transfers given to types below $r$. The Lagrangian can be written as

$$
\mathcal{L}=m(\theta)\left(u_{r}(a)+v_{r}(a)\right)-k \theta-\sum_{j=1}^{r-1} \lambda_{j r}\left(m(\theta)\left(u_{j}(a)+v_{r}(a)\right)-k \theta-\bar{U}_{j}-\epsilon\right) .
$$

By assumptions in part (i), (ii) and (iii) in Theorem 2, all requirements of envelope theorem are satisfied (as stated in Theorem 1 in Milgrom and Segal (2002)), so

$$
\frac{\partial \mathcal{L}}{\partial \epsilon}=\sum_{j=1}^{r-1} \lambda_{j r}
$$

We need to ensure that if $\epsilon>0$ is sufficiently close to 0 , no type is worse off and some types are strictly better off than equilibrium. Types $1,2, \ldots, r-1$ are strictly better off by the amount of $\epsilon-\frac{\sum_{j=1}^{r-1} \pi_{j}}{\sum_{j=1}^{r} \pi_{j}} \epsilon$. Types $r+1, r+2, \ldots, I$ receive the same payoff as in equilibrium. Finally, type $r$ receives

$$
\bar{U}_{r}(\epsilon)-\tilde{\epsilon} \approx \bar{U}_{r}(0)+\epsilon \frac{\partial \mathcal{L}}{\partial \epsilon}-\frac{\sum_{j=1}^{r-1} \pi_{j}}{\sum_{j=1}^{r} \pi_{j}} \epsilon=\bar{U}_{r}(0)+\underbrace{\left(\sum_{j=1}^{r-1} \lambda_{j r}-\frac{\sum_{j=1}^{r-1} \pi_{j}}{\sum_{j=1}^{r} \pi_{j}}\right) \epsilon}_{>0 \text { due to }(1)}
$$

so if $\epsilon>0$ is chosen sufficiently small, type $r$ will be weakly better off.

In the proposed mechanism, PC is satisfied, because no type is worse off than in equilibrium and $\mathrm{PC}$ is satisfied in equilibrium. Budget-balance is obviously satisfied by construction of $\tilde{\epsilon}$. The line of argument for verifying IC constraints is exactly the same as that in the proof of Theorem 1, so I do not repeat it here. Briefly speaking, all types are strictly worse off if they report a lower type in the equilibrium allocation, according to Lemma 3. Thus, if $\epsilon$ is sufficiently small, the proposed allocation satisfies IC constraints. Reporting higher types is not profitable either owing to the construction method.

Proof of Theorem 3. I construct a feasible mechanism in which type $i$ sellers match with probability $m\left(\theta_{i}^{F B}\right)$ and produce $a_{i}^{F B}$ for all $i$.

Under Assumption 2(i) and 4(i) or 4(ii), it can be easily shown that $U_{i}^{F B}$ is increasing in $i$. Let $\hat{i}$ denote the highest type of sellers without gains from trade. Then all types 1,2 , ..., $\hat{i}$ should be inactive, i.e., matched with probability 0 . In this case, the same construction method can be used to establish the proof with only one adjustment: the lowest type who should receive a positive payoff is the type $\hat{i}+1$. Since this adjustment is straightforward, to make the exposition simpler, I assume without loss of generality that there are positive gains from trade for all types, i.e., $U_{i}^{F B}>0$ for all $i$, and then I construct a feasible direct mechanism that achieves the first best for all these types.

Define $K_{i}(\theta, a) \equiv m(\theta) u_{i}(a), x \equiv(\theta, a)$ and $x_{i}^{*} \equiv\left(\theta_{i}^{*}, a_{i}^{*}\right)$. Construct the direct mecha$\operatorname{nism}\left\{\left(\theta_{i}^{*}, a_{i}^{*}, s_{i}^{*}\right)\right\}_{i \in\{1,2, \ldots, I\}}$ as follows: $\left(\theta_{i}^{*}, a_{i}^{*}\right)=\left(\theta_{i}^{F B}, a_{i}^{F B}\right)$ for all $i, s_{1}^{*}=-m\left(\theta_{1}^{*}\right) u_{1}\left(a_{1}^{*}\right)+s_{0}$, $s_{i}^{*}$ is defined recursively for $i>1$ :

$$
m\left(\theta_{i}^{*}\right) u_{i}\left(a_{i}^{*}\right)+s_{i}^{*}=m\left(\theta_{i-1}^{*}\right) u_{i}\left(a_{i-1}^{*}\right)+s_{i-1}^{*},
$$


and

$$
s_{0} \equiv \sum_{i=1}^{I} \pi_{i}\left[m\left(\theta_{i}^{*}\right)\left(v_{i}\left(a_{i}^{*}\right)+u_{i}\left(a_{i}^{*}\right)\right)-k \theta_{i}^{*}-\sum_{j=2}^{i}\left[K_{j}\left(\theta_{j-1}^{*}, a_{j-1}^{*}\right)-K_{j-1}\left(\theta_{j-1}^{*}, a_{j-1}^{*}\right)\right]\right] .
$$

The proposed allocation achieves the maximum welfare, so one simply needs to verify conditions for feasibility.

\section{Incentive Compatibility of Sellers}

I prove that this condition is satisfied in four steps. Figure 8 illustrates the steps used to establish the global incentive compatibility.

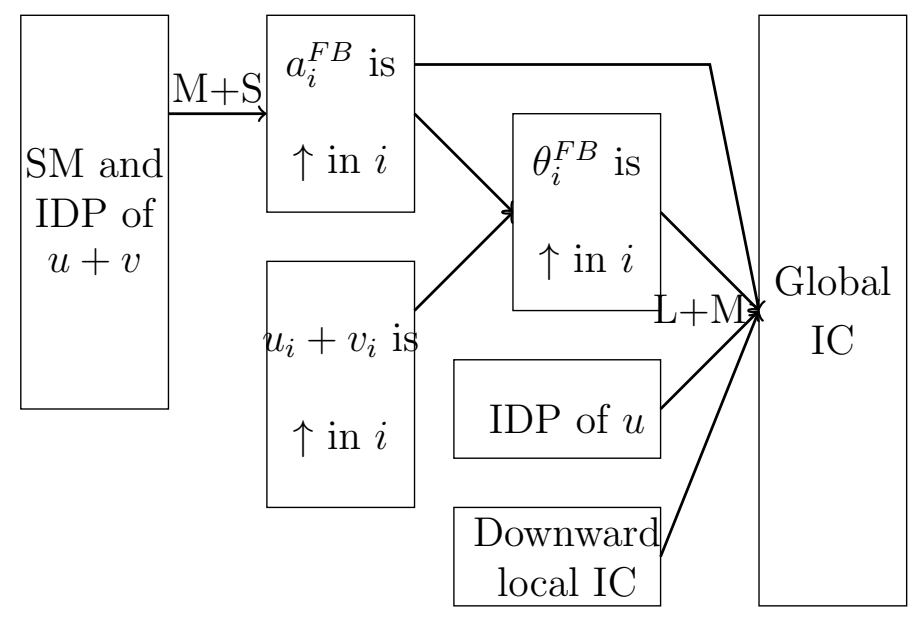

Figure 8: Schematic diagram indicating the proof steps of Theorem 3. SM and IDP refer to supermodularity and increasing differences property, respectively. M+S refers to Milgrom and Shannon (1994), $\mathrm{L}+\mathrm{M}$ refers to Laffont and Martimort (2009).

Step 1: $\left(\theta_{i}^{*}, a_{i}^{*}\right)$ is increasing in $i$.

First, $a_{i}^{*}=a_{i}^{F B}$ is increasing in $i$ because $u_{i}(a)+v_{i}(a)$ is super-modular in $a$ and has increasing differences in $(a ; i)$ under Assumption 3 (according to Theorem 5 in Milgrom and Shannon (1994)). Furthermore, $m(\theta)\left(u_{i}\left(a_{i}^{F B}\right)+v_{i}\left(a_{i}^{F B}\right)\right)-k \theta$ satisfies increasing differences property in $(\theta ; i)$ for the following reason. Suppose $\theta^{\prime}>\theta$ and $i>j$, then $m\left(\theta^{\prime}\right) f_{i}\left(a_{i}^{F B}\right)-$ $m(\theta) f_{i}\left(a_{i}^{F B}\right) \geq m\left(\theta^{\prime}\right) f_{j}\left(a_{j}^{F B}\right)-m(\theta) f_{j}\left(a_{j}^{F B}\right)$ is equivalent to $\left(f_{i}\left(a_{i}^{F B}\right)-f_{i}\left(a_{j}^{F B}\right)\right)+\left(f_{i}\left(a_{j}^{F B}\right)-\right.$ $\left.f_{j}\left(a_{j}^{F B}\right)\right) \geq 0$ since $m$ is increasing. But the latter is true because $a_{i}^{F B}$ is a maximizer of $\max _{a} f_{i}(a)$ so $f_{i}\left(a_{i}^{F B}\right) \geq f_{i}\left(a_{j}^{F B}\right)$, and $f_{i}(a)$ is increasing in $i$ under Assumption 2(i) and 4(i) or under Assumption 4(ii), so $f_{i}\left(a_{j}^{F B}\right) \geq f_{j}\left(a_{j}^{F B}\right)$. Consequently, $\theta_{i}^{*}=\theta_{i}^{F B}$ is increasing in $i$.

\section{Step 2: Local IC constraints are satisfied.}

Construction of $s_{i}^{*}$ is such that all local downward incentive compatibility constraints are satisfied and binding. That is, type $i$ is indifferent between reporting $i$ and $i-1$ for all $i>1$. Now I show that all local upward incentive compatibility constraints are also satisfied, i.e., 
type $i-1$ weakly prefers to report $i-1$ over $i$. The payoff to type $i-1$ by reporting $i$ is:

$$
\begin{aligned}
& m\left(\theta_{i}^{*}\right) u_{i-1}\left(a_{i}^{*}\right)+s_{i}^{*}=m\left(\theta_{i-1}^{*}\right) u_{i-1}\left(a_{i-1}^{*}\right)+s_{i-1}^{*}+m\left(\theta_{i-1}^{*}\right)\left(u_{i}\left(a_{i-1}^{*}\right)-u_{i-1}\left(a_{i-1}^{*}\right)\right)-m\left(\theta_{i}^{*}\right)\left(u_{i}\left(a_{i}^{*}\right)-u_{i-1}\left(a_{i}^{*}\right)\right) \\
& \leq m\left(\theta_{i-1}\right) u_{i-1}\left(a_{i-1}^{*}\right)+s_{i-1}^{*}+m\left(\theta_{i}^{*}\right)\left(u_{i}\left(a_{i-1}^{*}\right)-u_{i-1}\left(a_{i-1}^{*}\right)-u_{i}\left(a_{i}^{*}\right)+u_{i-1}\left(a_{i}^{*}\right)\right) \leq m\left(\theta_{i-1}^{*}\right) u_{i-1}\left(a_{i-1}^{*}\right)+s_{i-1}^{*}
\end{aligned}
$$

The first equality follows from the construction of $s_{i}^{*}$. The first inequality follows from the fact that $\theta_{i}^{*}$ and $u_{i}($.$) are both increasing in i$. The second inequality follows from increasing differences property of $u_{i}$ in $(a ; i)$ and also from the fact that $a_{i-1}^{*} \leq a_{i}^{*}$.

\section{Step 3: Other upward IC constraints are satisfied.}

I show that type $r$ does not gain by reporting $i$ for all $r$ and $i$ with $r<i$ :

$$
\begin{gathered}
m\left(\theta_{r}^{*}\right) u_{r}\left(a_{r}^{*}\right)+s_{r}^{*}-\left(m\left(\theta_{i}^{*}\right) u_{r}\left(a_{i}^{*}\right)+s_{i}^{*}\right) \\
=\sum_{j=r}^{i-1}\left[m\left(\theta_{j}^{*}\right) u_{j}\left(a_{j}^{*}\right)+s_{j}^{*}-\left(m\left(\theta_{j+1}^{*}\right) u_{j}\left(a_{j+1}^{*}\right)+s_{j+1}^{*}\right)+K_{j}\left(x_{j+1}^{*}\right)-K_{j}\left(x_{j}^{*}\right)-K_{r}\left(x_{j+1}^{*}\right)+K_{r}\left(x_{j}^{*}\right)\right] \\
\geq \sum_{j=r}^{i-1}\left[K_{j}\left(x_{j+1}^{*}\right)-K_{j}\left(x_{j}^{*}\right)-K_{r}\left(x_{j+1}^{*}\right)+K_{r}\left(x_{j}^{*}\right)\right] \\
\geq \sum_{j=r}^{i-1}\left[m\left(\theta_{j}^{*}\right)\left(u_{j}\left(a_{j+1}^{*}\right)-u_{j}\left(a_{j}^{*}\right)-u_{r}\left(a_{j+1}^{*}\right)+u_{r}\left(a_{j}^{*}\right)\right)\right] \geq 0 .
\end{gathered}
$$

The first equality is derived by using the telescoping technique. The first inequality uses the fact that type $i-1$ weakly prefers to report $i-1$ over $i$ (see step 2 ). The second inequality uses $\theta_{j+1}^{*} \geq \theta_{j}^{*}$ and also the fact that $u_{i}$ is increasing in $i$ for $a \in \bar{A}$. The last inequality is implied by the increasing differences property of $u$ and the fact that $a_{j+1}^{*} \geq a_{j}^{*}$.

\section{Step 4: Other downward IC constraints are satisfied.}

Similarly as above, I show that type $r$ does not gain by reporting $i<r$ :

$$
\begin{gathered}
m\left(\theta_{r}^{*}\right) u_{r}\left(a_{r}^{*}\right)+s_{r}^{*}-\left(m\left(\theta_{i}^{*}\right) u_{r}\left(a_{i}^{*}\right)+s_{i}^{*}\right) \\
=\sum_{j=i+1}^{r}\left[m\left(\theta_{j}^{*}\right) u_{j}\left(a_{j}^{*}\right)+s_{j}^{*}-\left(m\left(\theta_{j-1}^{*}\right) u_{j}\left(a_{j-1}^{*}\right)+s_{j-1}^{*}\right)+K_{j}\left(x_{j-1}^{*}\right)-K_{j}\left(x_{j}^{*}\right)-K_{r}\left(x_{j-1}^{*}\right)+K_{r}\left(x_{j}^{*}\right)\right] \\
\geq \sum_{j=i+1}^{r}\left[K_{j}\left(x_{j-1}^{*}\right)-K_{j}\left(x_{j}^{*}\right)-K_{r}\left(x_{j-1}^{*}\right)+K_{r}\left(x_{j}^{*}\right)\right] \\
\geq \sum_{j=i+1}^{r}\left[m\left(\theta_{j-1}^{*}\right)\left(u_{j}\left(a_{j-1}^{*}\right)-u_{j}\left(a_{j}^{*}\right)-u_{r}\left(a_{j-1}^{*}\right)+u_{r}\left(a_{j}^{*}\right)\right)\right] \geq 0 .
\end{gathered}
$$

The reasoning is exactly the same as that in step 3 and thus omitted here.

PC constraints, $U_{i}^{*} \equiv m\left(\theta_{i}^{*}\right) u_{i}\left(a_{i}^{*}\right)+s_{i}^{*} \geq 0$ for all $i$ 
$\mathrm{PC}$ is verified by induction. For $i=1$, I show below that $U_{1}^{*} \geq 0$. For $i>1$, note that $U_{i+1}^{*}=K_{i+1}\left(x_{i+1}^{*}\right)+s_{i+1}^{*}=K_{i+1}\left(x_{i}^{*}\right)+s_{i}^{*}=U_{i}^{*}+K_{i+1}\left(\theta_{i}^{*}, a_{i}^{*}\right)-K_{i}\left(\theta_{i}^{*}, a_{i}^{*}\right) \geq 0$ where the second equality holds because of the construction of $s_{i+1}^{*}$ and the inequality holds by the induction hypothesis and due to the fact that $u_{i}$ is increasing in $i$.

But $U_{1}^{*}=s_{0}$ because of the construction of $s_{1}^{*}$, so the important step is to show that $s_{0} \geq 0$. I consider two cases separately. In the first case, I prove it under Assumption 4(a), and in the second case, under Assumptions 4(ii) and 4(iii) .

Case 1: $s_{0} \geq 0$ under Assumption 4(i).

I show that each term in the RHS of (20) is positive. That is, $m\left(\theta_{i}^{F B}\right)\left(v_{i}\left(a_{i}^{F B}\right)+u_{i}\left(a_{i}^{F B}\right)\right)-$ $k \theta_{i}^{F B}-\sum_{j=2}^{i}\left[K_{j}\left(\theta_{j-1}^{F B}, a_{j-1}^{F B}\right)-K_{j-1}\left(\theta_{j-1}^{F B}, a_{j-1}^{F B}\right)\right] \geq 0$ for all $i$. I proceed by induction on $i$.

For $i=1$, the term is positive because $U_{1}^{F B} \geq 0$.

For $i=2$, one can write: $m\left(\theta_{2}^{F B}\right)\left(v_{2}\left(a_{2}^{F B}\right)+u_{2}\left(a_{2}^{F B}\right)\right)-k \theta_{2} \geq m\left(\theta_{1}^{F B}\right)\left(v_{2}\left(a_{1}^{F B}\right)+\right.$ $\left.u_{2}\left(a_{1}^{F B}\right)\right)-k \theta_{1}^{F B} \geq m\left(\theta_{1}^{F B}\right)\left(u_{2}\left(a_{1}^{F B}\right)-u_{1}\left(a_{1}^{F B}\right)\right)+m\left(\theta_{1}^{F B}\right)\left(v_{1}\left(a_{1}^{F B}\right)+u_{1}\left(a_{1}^{F B}\right)\right)-k \theta_{1}^{F B} \geq$ $K_{2}\left(\theta_{1}^{F B}, a_{1}^{F B}\right)-K_{1}\left(\theta_{1}^{F B}, a_{1}^{F B}\right)$. The first inequality holds true due to the fact that $\theta_{1}^{F B}$ and $a_{1}^{F B}$ are feasible for the type 2 maximization problem $\left.\max _{\theta, a}\left\{m(\theta)\left(v_{2}(a)+u_{2}(a)\right)-k \theta\right\}\right)$. The second one holds because $v_{i}($.$) is increasing in i$. The third one holds because $U_{1}^{F B} \geq 0$.

For $i \geq 2$, one has $m\left(\theta_{i}^{F B}\right)\left(v_{i}\left(a_{i}^{F B}\right)+u_{i}\left(a_{i}^{F B}\right)\right)-k \theta_{i}^{F B} \geq m\left(\theta_{i-1}^{F B}\right)\left(v_{i}\left(a_{i-1}^{F B}\right)+u_{i}\left(a_{i-1}^{F B}\right)\right)-k \theta_{i-1}^{F B}$ $\geq m\left(\theta_{i-1}^{F B}\right)\left(v_{i-1}\left(a_{i-1}^{F B}\right)+u_{i-1}\left(a_{i-1}^{F B}\right)\right)-k \theta_{i-1}^{F B}+m\left(\theta_{i-1}^{F B}\right)\left(u_{i}\left(a_{i-1}^{F B}\right)-u_{i-1}\left(a_{i-1}^{F B}\right)\right)$

$$
\begin{gathered}
\geq \sum_{j=2}^{i-1}\left[K_{j}\left(\theta_{j-1}^{F B}, a_{j-1}^{F B}\right)-K_{j-1}\left(\theta_{j-1}^{F B}, a_{j-1}^{F B}\right)\right]+K_{i}\left(\theta_{i-1}^{F B}, a_{i-1}^{F B}\right)-K_{i-1}\left(\theta_{i-1}^{F B}, a_{i-1}^{F B}\right) \\
=\sum_{j=2}^{i}\left[K_{j}\left(\theta_{j-1}^{F B}, a_{j-1}^{F B}\right)-K_{j-1}\left(\theta_{j-1}^{F B}, a_{j-1}^{F B}\right)\right]
\end{gathered}
$$

where the reasoning is exactly similar to the case of $i=2$.

Case 2: $s_{0} \geq 0$ under Assumptions 4(ii) and 4(iii).

I cannot show here that the terms inside the sigma in (20) are positive for each $i$, so I simplify the summation algebraically:

$$
\begin{aligned}
& s_{0}=\sum_{i=1}^{I} \pi_{i}\left[m\left(\theta_{i}^{F B}\right)\left(v_{i}\left(a_{i}^{F B}\right)+u_{i}\left(a_{i}^{F B}\right)\right)-k \theta_{i}^{F B}\right]-\sum_{i=1}^{I} \pi_{i}\left[\sum_{j=2}^{i}\left[K_{j}\left(\theta_{j-1}^{F B}, a_{j-1}^{F B}\right)-K_{j-1}\left(\theta_{j-1}^{F B}, a_{j-1}^{F B}\right)\right]\right] \\
& =\sum_{i=1}^{I} \pi_{i}\left[m\left(\theta_{i}^{F B}\right)\left(v_{i}\left(a_{i}^{F B}\right)+u_{i}\left(a_{i}^{F B}\right)\right)-k \theta_{i}^{F B}-\left[K_{i+1}\left(\theta_{i}^{F B}, a_{i}^{F B}\right)-K_{i}\left(\theta_{i}^{F B}, a_{i}^{F B}\right)\right] \frac{\Pi_{i}}{\pi_{i}}\right] \geq 0 .
\end{aligned}
$$

For the second equality, I changed the order of summations for the double sigma term and then used the definition of $\Pi_{i}$. Assumption 4(iii) is used to establish the last inequality.

\section{Planner's budget-balance condition}

It is algebraically easy to verify this condition due to the construction of $s_{0}$. 


\section{Proof of Theorem 4.}

\section{Step 1: Constructing a relaxed problem.}

First, I maximize the planner's objective function given only the budget-balance condition and local downward IC constraints. Remember the planner's original problem:

$$
\max _{\left\{\left(\theta_{i}, a_{i}, s_{i}\right)\right\}_{i \in\{1,2, \ldots, I\}}} \sum_{i=1}^{I} \pi_{i}\left(m\left(\theta_{i}\right) u_{i}\left(a_{i}\right)+s_{i}\right) \text { subject to: IC, PC and BB with equality. }
$$

BB with equality can be written as $\sum_{i=1}^{I} \pi_{i}\left[m\left(\theta_{i}\right)\left(u_{i}\left(a_{i}\right)+v_{i}\left(a_{i}\right)\right)-k \theta_{i}\right]=\sum_{i=1}^{I} \pi_{i}\left[m\left(\theta_{i}\right) u_{i}\left(a_{i}\right)+\right.$ $s_{i}$ ]. Therefore, the planner's problem is equivalent to:

$$
\max _{\left\{\left(a_{i}, s_{i}, \theta_{i}\right)\right\}_{i \in\{1,2, \ldots, I\}}} \sum_{i=1}^{I} \pi_{i}\left[m\left(\theta_{i}\right)\left(u_{i}\left(a_{i}\right)+v_{i}\left(a_{i}\right)\right)-k \theta_{i}\right]
$$

subject to: IC, PC, and $\sum_{i=1}^{I} \pi_{i}\left[m\left(\theta_{i}\right)\left(u_{i}\left(a_{i}\right)+v_{i}\left(a_{i}\right)\right)-k \theta_{i}\right]=\sum_{i=1}^{I} \pi_{i} U_{i}$.

To remind the reader of the notation, we have $\Pi_{i} \equiv \sum_{j=i+1}^{I} \pi_{j}, U_{i} \equiv m\left(\theta_{i}\right) u_{i}\left(a_{i}\right)+s_{i}$, $x_{i}=\left(\theta_{i}, a_{i}\right), K_{i}(\theta, a) \equiv m(\theta) u_{i}(a)$, so $K_{i}\left(x_{j}\right) \equiv m\left(\theta_{j}\right) u_{i}\left(a_{j}\right)$.

Now I use local downward IC constraints to simplify the last constraint in the problem:

$$
\sum_{i=1}^{I} \pi_{i} U_{i}=U_{1}+\sum_{i=1}^{I} \Pi_{i}\left(U_{i+1}-U_{i}\right) \geq U_{1}+\sum_{i=1}^{I} \Pi_{i}\left(K_{i+1}\left(x_{i}\right)-K_{i}\left(x_{i}\right)\right) \geq \sum_{i=1}^{I} \Pi_{i}\left(K_{i+1}\left(x_{i}\right)-K_{i}\left(x_{i}\right)\right),
$$

where the equality is derived by using the telescoping technique. The first inequality is implied by the local downward IC constraint: $U_{i+1}=K_{i+1}\left(x_{i+1}\right)+s_{i+1} \geq K_{i+1}\left(x_{i}\right)+s_{i}=$ $K_{i+1}\left(x_{i}\right)+U_{i}-K_{i}\left(x_{i}\right)$, so $U_{i+1}-U_{i} \geq K_{i+1}\left(x_{i}\right)-K_{i}\left(x_{i}\right)$. The last inequality follows from $\mathrm{PC}$ for type 1. Therefore, if BB together with local downward IC constraints and PC for type 1 are satisfied, then the following should hold: $\sum_{i=1}^{I} \pi_{i}\left(m\left(\theta_{i}\right)\left(u_{i}\left(a_{i}\right)+v_{i}\left(a_{i}\right)\right)-k \theta_{i}\right.$ $\geq \sum_{i=1}^{I} \Pi_{i}\left(K_{i+1}\left(\theta_{i}, a_{i}\right)-K_{i}\left(\theta_{i}, a_{i}\right)\right)$. Taking into account only the above constraint, a more relaxed problem is given by:

$$
\max _{\left\{\left(\theta_{i}, a_{i}\right)\right\}_{i \in\{1,2, \ldots, I\}}} \sum_{i=1}^{I} \pi_{i}\left[m\left(\theta_{i}\right)\left(u_{i}\left(a_{i}\right)+v_{i}\left(a_{i}\right)\right)-k \theta_{i}\right]
$$

subject to: $\sum_{i=1}^{I} \pi_{i}\left[m\left(\theta_{i}\right)\left(u_{i}\left(a_{i}\right)+v_{i}\left(a_{i}\right)\right)-k \theta_{i}-\left(K_{i+1}\left(\theta_{i}, a_{i}\right)-K_{i}\left(\theta_{i}, a_{i}\right)\right) \frac{\Pi_{i}}{\pi_{i}}\right] \geq 0$.

\section{Step 2: Finding necessary conditions for optimality.}

Let $\left\{\left(\theta_{i}^{\prime}, a_{i}^{\prime}\right)\right\}_{i \in\{1,2, \ldots, I\}}$ denote the solution to the relaxed problem. The Lagrangian for this problem can be written as $\mathcal{L}=\sum_{i=1}^{I} \pi_{i} \mathcal{L}_{i}\left(\theta_{i}, a_{i}, \zeta\right)$ where

$$
\mathcal{L}_{i}(\theta, a, \zeta) \equiv\left(m(\theta)\left(u_{i}(a)+v_{i}(a)\right)-k \theta_{i}\right)(1+\zeta)-\zeta m(\theta)\left(u_{i+1}(a)-u_{i}(a)\right) \frac{\Pi_{i}}{\pi_{i}},
$$


and $\zeta$ is the Lagrangian multiplier associated with the constraint. FOC is necessary at the optimal solution, so

$$
\begin{aligned}
\frac{\partial \mathcal{L}_{i}\left(\theta_{i}^{\prime}, a_{i}^{\prime}, \zeta^{*}\right)}{\partial \theta}=0 \text { and } \frac{\partial \mathcal{L}_{i}\left(\theta_{i}^{\prime}, a_{i}^{\prime}, \zeta^{*}\right)}{\partial a^{l}}=0 \text { for all } i \in\{1,2, \ldots, I\} \text { and } l \in\{1,2, \ldots, n\} \\
\sum_{i=1}^{I} \pi_{i} \mathcal{L}_{i}\left(\theta_{i}^{\prime}, a_{i}^{\prime}, \zeta^{*}\right) \geq 0 \text { with equality if } \zeta^{*}>0,
\end{aligned}
$$

with the Lagrangian at the optimal solution denoted by $\zeta^{*}$. In the lemma below, I show that any optimal mechanism is a solution to the relaxed problem, so the claim is established.

Lemma 4. Suppose Assumptions 2, 5 and 6 hold and all $u_{i}(a), v_{i}(a)$ and $m(\theta)$ are continuously differentiable. The optimal mechanism $\left\{\left(\theta_{i}^{*}, a_{i}^{*},.\right)\right\}$ solves (21) subject to (22).

Proof. I call the planner's problem with all IC, PC and BB constraints the original problem, and call (21) subject to (22) the relaxed problem. Denote the solution to the relaxed problem by $\left\{\left(\theta_{i}^{\prime}, a_{i}^{\prime}\right)\right\}_{i \in\{1,2, \ldots, I\}}$. The objective function is the same for both problems. The constraint set of the relaxed problem includes that of the original problem, so it is sufficient to show that for any solution to the relaxed problem, there exists a feasible mechanism associated with that. It will then follow that the value of objective function for both problems should be the same. As a result, $\left\{\left(\theta_{i}^{*}, a_{i}^{*}\right)\right\}_{i \in\{1,2, \ldots, I\}}$, an arbitrary solution to the original problem, must be associated with a solution to the relaxed problem, too.

Take an arbitrary solution to the relaxed problem, $\left\{\left(\theta_{i}^{\prime}, a_{i}^{\prime}\right)\right\}_{i \in\{1,2, \ldots, I\}}$, and set $s_{0} \equiv$ $\sum_{j=1}^{I} \pi_{i}\left[m\left(\theta_{i}^{\prime}\right)\left(v_{i}\left(a_{i}^{\prime}\right)+u_{i}\left(a_{i}^{\prime}\right)\right)-k \theta_{i}^{\prime}-\sum_{j=2}^{i}\left[K_{j}\left(\theta_{j-1}^{\prime}, a_{j-1}^{\prime}\right)-K_{j-1}\left(\theta_{j-1}^{\prime}, a_{j-1}^{\prime}\right)\right]\right], s_{1}^{\prime}=-m\left(\theta_{1}^{\prime}\right) u_{1}\left(a_{1}^{\prime}\right)+$ $s_{0}$, and for $i \geq 2$ :

$$
m\left(\theta_{i}^{\prime}\right) u_{i}\left(a_{i}^{\prime}\right)+s_{i}^{\prime}=m\left(\theta_{i-1}^{\prime}\right) u_{i}\left(a_{i-1}^{\prime}\right)+s_{i-1}^{\prime},
$$

or equivalently,

$$
m\left(\theta_{i}^{\prime}\right) u_{i}\left(a_{i}^{\prime}\right)+s_{i}^{\prime}=m\left(\theta_{1}^{\prime}\right) u_{1}\left(a_{1}^{\prime}\right)+s_{1}+\sum_{j=2}^{i}\left[K_{j}\left(x_{j-1}^{\prime}\right)-K_{j-1}\left(x_{j-1}^{\prime}\right)\right]
$$

where $x_{i}^{\prime} \equiv\left(\theta_{i}^{\prime}, a_{i}^{\prime}\right)$.

Claim 1: Mechanism $\left\{\left(\theta_{i}^{\prime}, a_{i}^{\prime}, s_{i}^{\prime}\right)\right\}_{i \in\{1,2, \ldots, I\}}$ satisfies PC in the original problem.

$\mathrm{PC}$ is verified by a simple induction. For $i=1, U_{1}=s_{0} \geq 0$ from (22). For $i>1$ : $U_{i+1}=U_{i}+K_{i+1}\left(\theta_{i}^{\prime}, a_{i}^{\prime}\right)-K_{i}\left(\theta_{i}^{\prime}, a_{i}^{\prime}\right) \geq 0$, where the inequality holds by induction hypothesis and the fact that $u_{i}$ is increasing in $i$.

Claim 2: Mechanism $\left\{\left(\theta_{i}^{\prime}, a_{i}^{\prime}, s_{i}^{\prime}\right)\right\}_{i \in\{1,2, \ldots, I\}}$ satisfies IC constraints in the original problem.

IC constraints are verified in claims 2-a to 2-d:

Claim 2-a: $\left(\theta_{i}^{\prime}, a_{i}^{\prime}\right)$ is increasing in $i$.

$\left(\theta_{i}^{\prime}, a_{i}^{\prime}\right)$ satisfies FOC of the relaxed problem, specially the first one. Therefore, $a_{i}^{\prime}$ is increasing in $i$ because $r_{i}^{\rho}(a)$ is super-modular in $a$ and has increasing differences in $(a ; i)$ 
by Assumption 5 (according to Theorem 5 in Milgrom and Shannon (1994)), where $\rho=$ $\frac{\zeta}{\zeta+1} \in[0,1]$. Furthermore, $\left\{\left(m(\theta)\left(u_{i}(a)+v_{i}(a)\right)-k \theta\right)(1+\zeta)-\zeta\left(K_{i+1}\left(\theta_{i}, a_{i}\right)-K_{i}\left(\theta_{i}, a_{i}\right)\right) \frac{\Pi_{i}}{\pi_{i}}\right\}$, which is equal to $\left(m(\theta) r_{i}^{\rho}\left(a_{i}^{\prime}\right)-k \theta\right)(1+\zeta)$, satisfies increasing differences property in $(\theta ; i)$ for the same reason as in step 1 in Theorem 3 (only $f_{i}$ should be replaced by $r_{i}^{\rho}$ ). Hence, $\theta_{i}^{\prime} \equiv \arg \max _{\theta}\left(m(\theta) r_{i}^{\rho}\left(a_{i}^{\prime}\right)-k \theta\right)$, is increasing in $i$.

Claim 2-b: Local IC constraints are satisfied.

Claim 2-c: Other upward IC constraints are satisfied.

Claim 2-d: Other downward IC constraints are satisfied.

Since $s_{i}^{\prime}$ has been recursively constructed similar to the construction of $s_{i}^{*}$ in the proof of Theorem 3, and since $\left(\theta_{i}^{\prime}, a_{i}^{\prime}\right)$ is increasing in $i$, claims 2-b to 2-d can be proved exactly as in steps 2 to 4 in the aforementioned proof, thus they are omitted here.

Claim 3: Mechanism $\left\{\left(\theta_{i}^{\prime}, a_{i}^{\prime}, s_{i}^{\prime}\right)\right\}_{i \in\{1,2, \ldots, I\}}$ satisfies BB in the original problem. It is algebraically easy to verify $\mathrm{BB}$ following from the constraint in the relaxed problem and because of the construction of $\left\{s_{i}^{\prime}\right\}_{i \in\{1,2, \ldots, I\}}$.

\section{Proofs of Section 4}

I suppress superscript * for the proofs in the asset market with lemons to make the notation simpler, unless there is a danger of confusion.

Proof of Proposition 1: Optimal mechanism if $\pi_{1} b_{1}+\pi_{2} b_{2} \geq c_{2}$. Consider the following direct mechanism: $\left\{\left(\theta_{i}^{*}, a_{i}^{*}, s_{i}^{*}\right)\right\}_{i \in\{1,2\}}$ with $a_{i}^{*}=1, s_{i}^{*}=\pi_{1} b_{1}+\pi_{2} b_{2}$, $\theta_{i}^{*}=1$ for all $i$. Incentive compatibility of sellers is clearly satisfied, because both types receive the same $\left(\theta_{i}, a_{i}, s_{i}\right)$. Also, $\mathrm{PC}$ and $\mathrm{BB}$ can be easily verified. The objective function is maximized because the $\theta$ and $a$ allocated to both types are the same as what they receive under complete information.

Proof of Proposition 1: Optimal mechanism if $\pi_{1} b_{1}+\pi_{2} b_{2}<c_{2}$.

Here, the planner cannot achieve the first best by using a pooling allocation, because type two gets a strictly negative payoff in the pooling allocation. It is not possible to use Theorem 3 , because if $b_{2}-c_{2}>b_{1}-c_{1}$, then Assumption 4(ii) is violated. If $b_{2}-c_{2} \leq b_{1}-c_{1}$, then Assumption 4 (iii) requires $\pi_{1} b_{1}+\pi_{2} b_{2} \geq c_{2}$. Therefore, the planner's problem shall be solved directly by taking all constraints into account.

\section{Step 1: Formulating the planner's problem.}

Let $\left\{\left(\theta_{i}, a_{i}, s_{i}\right)\right\}_{i \in\{1,2\}}$ denote the direct mechanism. As explained before, the planner's budget-balance condition must be binding, so $\sum_{i=1}^{2} \pi_{i} s_{i}=\sum_{i=1}^{2} \pi_{i}\left[\min \left\{\theta_{i}, 1\right\} a_{i} h_{i}-k \theta_{i}\right]$, thus the planner's problem can be summarized as follows:

Problem 3.

$$
\begin{gathered}
\max _{\left\{\left(\theta_{i}, a_{i}, s_{i}\right)\right\}_{i=1,2}} \sum_{i=1}^{2} \pi_{i}\left(\min \left\{\theta_{i}, 1\right\} a_{i}\left(h_{i}-c_{i}\right)-k \theta_{i}\right) \\
\text { subject to : } s_{1}-\min \left\{\theta_{1}, 1\right\} a_{1} c_{1} \geq s_{2}-\min \left\{\theta_{2}, 1\right\} a_{2} c_{1}(I C-12),
\end{gathered}
$$




$$
\begin{gathered}
s_{2}-\min \left\{\theta_{2}, 1\right\} a_{2} c_{2} \geq s_{1}-\min \left\{\theta_{1}, 1\right\} a_{1} c_{2}(I C-21), \\
s_{1}-\min \left\{\theta_{1}, 1\right\} a_{1} c_{1} \geq 0(P C-1), s_{2}-\min \left\{\theta_{2}, 1\right\} a_{2} c_{2} \geq 0(P C-2), \\
\sum_{i=1}^{2} \pi_{i}\left(\min \left\{\theta_{i}, 1\right\} a_{i} h_{i}-k \theta_{i}-s_{i}\right)=0(B B) .
\end{gathered}
$$

Step 2: $\theta_{1}>0$ and $\theta_{2}>0$.

If both $\theta_{1}$ and $\theta_{2}$ are 0 , then the welfare level equals 0 , but this is not possible because the equilibrium allocation is feasible and delivers a strictly positive value. To rule out the case that one of them is 0 , note that IC-12 and IC-21 together imply that:

$$
\left(m\left(\theta_{1}\right) a_{1}-m\left(\theta_{2}\right) a_{2}\right) c_{1} \leq s_{1}-s_{2} \leq\left(m\left(\theta_{1}\right) a_{1}-m\left(\theta_{2}\right) a_{2}\right) c_{2}
$$

But $c_{1}<c_{2}$, therefore,

$$
m\left(\theta_{1}\right) a_{1} \geq m\left(\theta_{2}\right) a_{2} .
$$

If $\theta_{1}=0$, then $\theta_{2}$ must be 0 as well, and this leads to 0 level of welfare, which is not possible as mentioned above, thus $\theta_{1}>0$. If $\theta_{2}=0$, then it is easy to check that the maximum possible welfare in this case, even if $\theta_{1}=1$, is less than the welfare level under the proposed solution. Therefore, $\theta_{2}>0$.

Step 3: $\theta_{1} \leq 1$ and $\theta_{2} \leq 1$.

Suppose $\theta_{i}>1$ for some $i$. Then consider the following: $\theta_{i}^{\prime}=1, s_{i}^{\prime}=s_{i}+k\left(\theta_{i}-1\right) \pi_{i}$ and $s_{j}^{\prime}=s_{j}+k\left(\theta_{i}-1\right) \pi_{i}$ where $j \neq i$. If $\left(\theta_{i}, s_{1}, s_{2}\right)$ is replaced by $\left(\theta_{i}^{\prime}, s_{1}^{\prime}, s_{2}^{\prime}\right)$, the new solution satisfies all the constraints: Obviously, IC-12 and IC-21 constraints are still satisfied, because the change in $s_{1}$ is the same as the change in $s_{2}$ and also $\min \left\{\theta_{1}, 1\right\}$ and $\min \left\{\theta_{2}, 1\right\}$ have not changed. PC-1 and PC-2 are satisfied because $s_{1}^{\prime}>s_{1}$ and $s_{2}^{\prime}>s_{2}$. BB is also satisfied by construction of $s_{1}^{\prime}$ and $s_{2}^{\prime}$. This is a contradiction because the value of the objective function has increased by $k\left(\theta_{i}-1\right)$. Therefore, for all $i \in\{1,2\}, \theta_{i} \leq 1$.

Step 4: $a_{1}=a_{2}=1$.

Suppose $a_{i}<1$ for some $i$. Let $a_{i}^{\prime} \equiv \frac{a_{i} \theta_{i}}{\theta_{i}-\epsilon}$ where $0<\epsilon<\theta_{i}\left(1-a_{i}\right)$. Fix $\epsilon$ and consider the following: $\theta_{i}^{\prime}=\theta_{i}-\epsilon, s_{i}^{\prime}=s_{i}+k \epsilon \pi_{i}$ and $s_{j}^{\prime}=s_{j}+k \epsilon \pi_{i}$ where $j \neq i$. Now, replace $\left(\theta_{i}, a_{i}, s_{1}, s_{2}\right)$ by $\left(\theta_{i}^{\prime}, a_{i}^{\prime}, s_{1}^{\prime}, s_{2}^{\prime}\right)$. The proposed solution satisfies all the constraints: Obviously, IC-12 and IC-21 constraints are still satisfied, because $\min \left\{\theta_{i}, 1\right\} a_{i}=\min \left\{\theta_{i}^{\prime}, 1\right\} a_{i}^{\prime} . \mathrm{PC}-1$ and PC-2 are satisfied because $s_{1}^{\prime}>s_{1}$ and $s_{2}^{\prime}>s_{2}$. BB is satisfied by construction of $s_{1}^{\prime}$ and $s_{2}^{\prime}$. This is a contradiction, because the value of the objective function has increased by $k \epsilon$. Therefore, $a_{i}=1$ for all $i \in\{1,2\}$. I write the planner's problem again incorporating the results so far:

\section{Problem 4.}

$$
\begin{gathered}
\max _{\left\{\left(\theta_{i}, s_{i}\right)\right\}_{i=1,2}} \sum_{1}^{2} \pi_{i}\left(\theta_{i}\left(h_{i}-c_{i}\right)-k \theta_{i}\right), \\
\text { s.t.: } s_{1}-\theta_{1} c_{1} \geq s_{2}-\theta_{2} c_{1}(I C-12), s_{2}-\theta_{2} c_{2} \geq s_{1}-\theta_{1} c_{2}(I C-21),
\end{gathered}
$$




$$
\begin{gathered}
s_{1}-\theta_{1} c_{1} \geq 0(P C-1), s_{2}-\theta_{2} c_{2} \geq 0(P C-2), \\
\sum_{1}^{2} \pi_{i}\left(\theta_{i} h_{i}-k \theta_{i}-s_{i}\right)=0(B B) .
\end{gathered}
$$

Step 5: $\theta_{1}=1$.

According to step $4, a_{1}=a_{2}=1$, so $\theta_{1} \geq \theta_{2}$ following (26). By way of contradiction, assume that at the solution $\theta_{1}<1$. Two cases are considered. First, assume that PC-2 is not binding. Consider the following: $\theta_{i}^{\prime}=\theta_{i}+\epsilon$ and $s_{i}^{\prime}=s_{i}+\left(\pi_{1} b_{1}+\pi_{2} b_{2}\right) \epsilon$ for all $i$ where $\epsilon \in\left(0, \min \left\{1-\theta_{1}, \frac{s_{2}-\theta_{2} c_{2}}{c_{2}-\pi_{1} b_{1}-\pi_{2} b_{2}}\right\}\right)$. It is easy to check that all constraints are satisfied, but the value of the objective function has now increased by $\left(\pi_{1}\left(b_{1}-c_{1}\right)+\pi_{2}\left(b_{2}-c_{2}\right)\right) \epsilon$, a contradiction. Note that (26) was used to ensure that $\theta_{2}+\epsilon<1$.

Second, assume that PC-2 is binding. Consider the following: $\theta_{1}^{\prime}=\theta_{1}+\epsilon$ where $\epsilon<1-\theta_{1}$, $s_{1}^{\prime}=s_{1}+b_{1} \epsilon$. It is again easy to check that all constraints are satisfied. The only tricky thing here is to check that IC-21 is satisfied: the LHS in IC-21 is fixed, but the RHS increases by $\epsilon\left(b_{1}-c_{2}\right)$, which is negative, so IC-21 is not violated. (Note that $b_{1}-c_{2}<0$, otherwise $\pi_{1} b_{1}+\pi_{2} b_{2}>\pi_{1} c_{2}+\pi_{2} c_{2}=c_{2}$, which contradicts the assumption.) The value of the objective function now has increased by $\pi_{1} b_{1} \epsilon$, a contradiction.

\section{Step 6: Calculating $\theta_{2}$ and the rest of unknowns.}

I write $s_{1}$ from the budget-balance condition in terms of other variables: $s_{1}=b_{1}+$ $\frac{\pi_{2}}{\pi_{1}} \theta_{2} b_{2}-\frac{\pi_{2}}{\pi_{1}} s_{2}$. After substituting $s_{1}$ from the above equation, (25) turns into:

$$
\left(1-\theta_{2}\right) c_{1} \leq b_{1}+\frac{\pi_{2}}{\pi_{1}} \theta_{2} b_{2}-\frac{s_{2}}{\pi_{1}} \leq\left(1-\theta_{2}\right) c_{2}
$$

First, note that PC-1 is implied by IC-12 and PC-2. Second, I argue that PC-2 must be binding at the solution. By way of contradiction, suppose it is not. Then only (27) is sufficient to determine $\theta_{2}$. But to maximize the objective function, I need to choose the highest possible $\theta_{2}$ consistent with (27), which is $\theta_{2}=1$. But according to (27), $s_{2}=\pi_{1} b_{1}+\pi_{2} b_{2}$ and $s_{2}>c_{2}$ from PC-2, which is a contradiction with $\pi_{1} b_{1}+\pi_{2} b_{2}<c_{2}$. Therefore, PC-2 must be binding. Third, since PC-2 is binding, I replace $s_{2}$ by $\theta_{2} c_{2}$ and rewrite (27) again:

$$
\left(1-\theta_{2}\right) c_{1} \leq b_{1}+\frac{\theta_{2}}{\pi_{1}}\left(\pi_{2} b_{2}-c_{2}\right) \leq\left(1-\theta_{2}\right) c_{2} \text {. }
$$

It is easy to see that the right inequality in (28) is satisfied for any $\theta_{2} \in[0,1]$, because $b_{1}<c_{2}$. To maximize the objective function, I need to find the maximum value for $\theta_{2}$ under which the left inequality in (28) is satisfied. This implies that

$$
\theta_{2}=\frac{\pi_{1}\left(b_{1}-c_{1}\right)}{c_{2}-\pi_{2} b_{2}-\pi_{1} c_{1}} .
$$

The proof is complete, because I have found the values for $a_{i}, \theta_{i}$ and $s_{i}{ }^{33}$

\footnotetext{
${ }^{33}$ To find an associated implementable allocation and policy, values of $p_{i}$ from $\theta_{i}$ and $s_{i}$ can be calculated to ensure that they are the same as those in Table 1 . Note that $t_{i}$ in Table 1 is calculated such that buyers' free-entry condition is satisfied for each submarket in the decentralized economy.
} 
Proof of Proposition 1: Pareto inefficiency of equilibrium if $\pi_{1} c_{1}+\pi_{2} b_{2} \geq c_{2}$. In equilibrium, $U_{1}^{E Q}=b_{1}-c_{1}$ and $U_{2}^{E Q}=\frac{b_{1}-c_{1}}{b_{2}-c_{1}}\left(b_{2}-c_{2}\right)$. If $\pi_{1} c_{1}+\pi_{2} b_{2} \geq c_{2}$, then $\pi_{1} b_{1}+\pi_{2} b_{2} \geq$ $c_{2}$. In the proposed allocation for the case of $\pi_{1} b_{1}+\pi_{2} b_{2} \geq c_{2}, U_{1}^{*}=\pi_{1} b_{1}+\pi_{2} b_{2}-c_{1}$, $U_{2}^{*}=\pi_{1} b_{1}+\pi_{2} b_{2}-c_{2}$. Therefore, if $\pi_{1} c_{1}+\pi_{2} b_{2} \geq c_{2}$, then $U_{1}^{E Q}<U_{1}^{*}$ and $U_{2}^{E Q} \leq U_{2}^{*}$. The proof is complete.

As an illustration of how the proof of Theorem 2 works, one can obtain the same sufficient condition for constrained Pareto inefficiency of equilibrium using Lagrangian as described below. GSW show that $a$ is always equal to 1, and I showed the same is true in the planner's problem, so I fix $a=1$ for both types. Type 1 solves the following problem in equilibrium: $\bar{U}_{1}=\max _{\theta, p} m(\theta)\left(p-c_{1}\right)$ subject to $q(\theta)\left(h_{1}-p\right) \geq k$. At the solution, $\theta_{1}^{E Q}=1, p_{1}^{E Q}=$ $h_{1}-k=b_{1}$ and $\bar{U}_{1}=b_{1}-c_{1}$. For type 2 , the problem is as follows, taking into account that the free-entry condition is always binding:

$$
\begin{gathered}
\max _{\theta}\left\{m(\theta)\left(h_{2}-c_{2}\right)-k \theta\right\} \\
\text { subject to } m(\theta)\left(h_{2}-c_{1}\right)-k \theta \leq \bar{U}_{1}+\epsilon,
\end{gathered}
$$

where $\epsilon=0$ in equilibrium. Here is the Lagrangian:

$$
\mathcal{L}=m(\theta)\left(h_{2}-c_{2}\right)-k \theta-\lambda_{1}\left(m(\theta)\left(h_{2}-c_{1}\right)-k \theta-\bar{U}_{1}-\epsilon\right) .
$$

The constraint is binding and $\theta<1$, so $m(\theta)$ is continuously differentiable in the neighborhood of the solution, thus FOC implies $h_{2}-c_{2}-k-\lambda_{1}\left(h_{2}-c_{1}-k\right)=0$. Therefore, $\lambda_{1}=\frac{b_{2}-c_{2}}{b_{2}-c_{1}}$ and $\theta_{2}=\frac{b_{1}-c_{1}+\epsilon}{b_{2}-c_{1}}$. In equilibrium, the allocation for type 2 is distorted. The planner relaxes the incentive constraint by paying $\epsilon>0$ to type 1 and taxing both types by $\pi_{1} \epsilon$. The required condition in Theorem 2 is that $\lambda_{1}=\frac{b_{2}-c_{2}}{b_{2}-c_{1}}>\frac{\pi_{1}}{\pi_{1}+\pi_{2}}=\pi_{1}$, which is equivalent to $c_{2}<\pi_{1} c_{1}+\pi_{2} b_{2}$. The payoff to type 2 net of transfers is:

$$
\frac{b_{1}-c_{1}+\epsilon}{b_{2}-c_{1}}\left(b_{2}-c_{2}\right)-\pi_{1} \epsilon=\frac{b_{1}-c_{1}}{b_{2}-c_{1}}\left(b_{2}-c_{2}\right)+\left(\frac{b_{2}-c_{2}}{b_{2}-c_{1}}-\pi_{1}\right) \epsilon>\bar{U}_{2}(0)=U_{2}^{E Q}
$$

for $\epsilon>0$, where the inequality holds true because $\lambda_{1}>\pi_{1}$.

No gains from trade for type 1, and $\pi_{1} b_{1}+\pi_{2} b_{2}-c_{2}<0$ imply $\theta_{1}=\theta_{2}=0$. I characterize the solution to the planner's problem when $b_{1}<c_{1}<c_{2}<b_{2}$ and $\pi_{1} b_{1}+\pi_{2} b_{2}-$ $c_{2}<0$. The proof is similar to the previous proof up to step 5 , so I do not repeat those steps here and begin from Problem 4. First, (26) implies that $\theta_{1} \geq \theta_{2}$. Second, PC- 1 is implied by IC-12 and PC-2, so PC- 1 can be ignored. Third, if $\theta_{1}=0$, then $0 \leq \theta_{2} \leq \theta_{1}=0$ and the proof is complete. Therefore, by way of contradiction, assume that $\theta_{1}>0$. I proceed in 4 steps. 
Step 1: $\theta_{2}<\theta_{1}$.

Suppose to the contrary that $\theta_{2} \geq \theta_{1}$, but $\theta_{2}$ cannot exceed $\theta_{1}$ as mentioned above, so $\theta_{2}=\theta_{1}$. IC-12 and IC-21 together imply that $s_{1}=s_{2}$. Then, BB implies that $s_{2}=$ $\left(\pi_{1} b_{1}+\pi_{2} b_{2}\right) \theta_{2}$. The latter together with PC-2 implies that $\left(\pi_{1} b_{1}+\pi_{2} b_{2}-c_{2}\right) \theta_{2} \geq 0$. But $\pi_{1} b_{1}+\pi_{2} b_{2}-c_{2}<0$, therefore, $\theta_{2}=0$, so $\theta_{1}=0$. This is a contradiction with $\theta_{1}>0$.

\section{Step 2: IC-21 is binding.}

By way of contradiction, suppose IC-21 is not binding. Consider $\theta_{1}^{\prime}=\theta_{1}-\epsilon$ and $s_{1}^{\prime}=$ $s_{1}-b_{1} \epsilon$ with $\epsilon>0$. Since $\theta_{1}>0$ and IC-21 is not binding, one can find a sufficiently small $\epsilon$ such that $\theta_{1}^{\prime}>0$ and IC-21 still holds using the fact that $b_{1}-c_{1}<0$. Now, it is easy to check that $\left\{\left(\theta_{1}^{\prime}, s_{1}^{\prime}\right),\left(\theta_{2}, s_{2}\right)\right\}$ is feasible for Problem 4 , but it leads to a higher value for the objective function than $\left\{\left(\theta_{1}, s_{1}\right),\left(\theta_{2}, s_{2}\right)\right\}$.

\section{Step 3: IC-12 is not binding.}

Suppose by way of contradiction that IC-12 is binding. Then, following step 2, it is easy to check that $s_{1}=s_{2}$ and $\theta_{1}=\theta_{2}$. Then BB implies that $s_{2}=\left(\pi_{1} b_{1}+\pi_{2} b_{2}\right) \theta_{2}$. According to PC-2, $\left(\pi_{1} b_{1}+\pi_{2} b_{2}-c_{2}\right) \theta_{2} \geq 0$. But $\pi_{1} b_{1}+\pi_{2} b_{2}-c_{2}<0$, so $\theta_{1}=\theta_{2}=0$. This is a contradiction, so IC-12 is not binding.

\section{Step 4: Getting a contradiction.}

To get a contradiction, consider $\left\{\left(\theta_{1}^{\prime}, s_{1}^{\prime}\right),\left(\theta_{2}, s_{2}^{\prime}\right)\right\}$ where $\theta_{1}^{\prime}=\theta_{1}-\epsilon$ with $\epsilon>0, s_{1}^{\prime}=$ $s_{1}-\left(\pi_{1} b_{1}+\pi_{2} b_{2}\right) \epsilon$ and $s_{2}^{\prime}=s_{2}+\pi_{1}\left(c_{2}-b_{1}\right) \epsilon$. Since $\theta_{1}>0$ and IC-12 is not binding, one can find a sufficiently small $\epsilon$ such that $\theta_{1}^{\prime}>0$ and IC-12 still holds. It is now easy to check that $\left\{\left(\theta_{1}^{\prime}, s_{1}^{\prime}\right),\left(\theta_{2}, s_{2}^{\prime}\right)\right\}$ is feasible for Problem 4 , but it leads to a higher value for the objective function than $\left\{\left(\theta_{1}, s_{1}\right),\left(\theta_{2}, s_{2}\right)\right\}$, a contradiction. The proof is complete.

\section{Proofs of Section 7}

First, I define a feasible mechanism that is exactly similar to its counterpart in the discrete type space, Definition 3. The planner allocates each seller a market tightness, $\tilde{\theta}: Z \rightarrow \mathbb{R}_{+}$, a transfer conditional on finding a match, $\tilde{p}: Z \rightarrow \mathbb{R}$, and an unconditional transfer, $t_{0} \in R_{+}$. Also, the planner charges each buyer who matches $\tilde{t}: Z \rightarrow \mathbb{R}$ units of the numeraire good based on the type of the seller that the buyer is matched with.

Definition 7 (Feasible Mechanism for Continuous Type Space). A feasible mechanism is a set $\left\{\left(\tilde{\theta}(),. \tilde{p}(),. \tilde{t}(),. \tilde{t}_{0}\right)\right\}$ such that the following conditions hold:

(i)Incentive compatibility of sellers: For all $z$ and $\hat{z}$,

$$
U(z) \equiv m(\tilde{\theta}(z))(\tilde{p}(z)-c(z))+\tilde{t}_{0} \geq U(z, \hat{z}) \equiv m(\tilde{\theta}(\hat{z}))(\tilde{p}(\hat{z})-c(z))+\tilde{t}_{0}
$$

(ii) Participation constraint of sellers: For all z,

$$
U(z) \geq 0
$$


(iii) Buyers' zero profit condition: For all z,

$$
q(\tilde{\theta}(z))(h(z)-\tilde{p}(z)-\tilde{t}(z))-k=0 .
$$

(iv) Planner's budget-balance condition

$$
\int m(\tilde{\theta}(z)) \tilde{t}(z) d F(z)-\tilde{t}_{0} \geq 0
$$

It is straightforward to see, and important to note, that although the definition of feasible mechanism here looks different than the direct mechanism in the discrete type space, but with a change in variable, $\tilde{s}(z) \equiv m(\tilde{\theta}(z)) \tilde{p}(z)+\tilde{t}_{0}$, and combining the buyers' zero profit condition with the planner's budget-balance condition, one will obtain a description of the mechanism completely analogous to that in Definition 3:

Definition 8 (Feasible mechanism for continuous type space-equivalent definition). A feasible mechanism is a set $\{(\tilde{\theta}(),. \tilde{s}())$.$\} such that the following conditions hold:$

(i) Incentive compatibility of sellers: For all $z$ and $\hat{z}$,

$$
U(z) \equiv-m(\tilde{\theta}(z)) c(z)+\tilde{s}(z) \geq U(z, \hat{z}) \equiv-m(\tilde{\theta}(\hat{z})) c(z)+\tilde{s}(\hat{z})
$$

(ii) Participation constraint of sellers: For all z,

$$
U(z) \geq 0
$$

(iii) Planner's budget-balance condition

$$
\int[m(\tilde{\theta}(z)) h(z)-k \tilde{\theta}(z)-\tilde{s}(z)] d F(z) \geq 0 .
$$

I still work with the first definition above since $p$ and $t$ have natural interpretations of submarket-specific price and sales tax, respectively.

Definition 9. An optimal mechanism is a feasible mechanism that maximizes the planner's objective function among all feasible mechanisms.

The implementable allocation with two types of taxes on buyers, not only sales tax but also entry tax, is defined here similarly to the definition of implementable allocation in Definition 5.

Definition 10 (Implementable allocation for continuous type space with sales and entry tax). An allocation, $\{G, \mathcal{P}, \theta, \mu\}$, is implementable through policy $\left\{t, t_{e}, t_{0}\right\}$ if the following conditions are satisfied:

(i) Buyers' profit maximization, free entry and no commitment For any $p \in \mathbb{P}$,

$$
q(\theta(p))\left[\int h(z) \mu(z \mid p) d z-t(p)\right] \leq k+t_{e}(p)
$$


with equality if $p \in \mathcal{P}$. Also, $0 \leq k+t_{e}(p)$ for any $p \in \mathcal{P}$.

(ii) Sellers' optimal search: the same as Definition 5(ii).

(iii) Feasibility or market clearing: the same as Definition and 5(iii),

(iv) Planner's budget-balance condition

$$
\int_{\mathcal{P}}\left[q(\theta(p)) t(p)+t_{e}(p)\right] d G(p) \geq t_{0} .
$$

Lemma 5 (Counterpart of Lemma 1 for continuous type space). Assume $c^{\prime}(z)>0$ for all $z$. Take any feasible mechanism in which all types receive a strictly positive payoff, and in which the market tightness allocated to different types is all different. Then there exists an associated implementable allocation under which all types receive exactly the same payoff as in the direct mechanism.

Proof of Lemma $5(i)$. Consider a feasible mechanism $\left\{\tilde{\theta}(),. \tilde{p}(),. \tilde{t}(),. \tilde{t}_{0}\right\}$. Denote by $U(z)$ the payoff to type $z$ in this mechanism. I construct an allocation $\{G, \mathcal{P}, \Theta, \mu\}$ as follows:

$$
\mathcal{P} \equiv\left[p_{L}, p_{H}\right] \subseteq \mathbb{P} \equiv R_{+} \text {where } p_{L} \equiv \tilde{p}\left(z_{L}\right) \text { and } p_{H} \equiv \tilde{p}\left(z_{H}\right)
$$

and $\tilde{p}$ is given by (9) (where $\tilde{\theta}$ should replace $\left.\tilde{\theta}^{*}\right) \cdot{ }^{34}$ Define $\bar{m}=\lim _{\theta \rightarrow \infty} m(\theta)$. The market tightness for this allocation is given by:

$$
\begin{gathered}
\begin{cases}\Theta(p)=\infty & \text { for } p \leq c\left(z_{L}\right) \\
m(\Theta(p))=\min \left\{\bar{m}, \frac{U\left(z_{L}\right)}{p-c\left(z_{L}\right)}\right\} & \text { for } p \in\left(c\left(z_{L}\right), p_{L}\right) \\
\Theta(p)=\tilde{\theta}\left(\tilde{p}^{-1}(p)\right) & \text { for } p \in\left[p_{L}, p_{H}\right] \\
m(\Theta(p))=\min \left\{\bar{m}, \frac{U\left(z_{H}\right)}{p-c\left(z_{H}\right)}\right\} & \text { for } p>p_{H}\end{cases} \\
G(p)= \begin{cases}0 & \text { for } p<p_{L} \\
\int_{p_{L}}^{p} \Theta(p) F^{\prime}\left(\tilde{p}^{-1}(p)\right) d p & \text { for } p \in\left[p_{L}, p_{H}\right] \\
1 & \text { for } p>p_{H}\end{cases} \\
\int \mu(z \mid p) d z=1 \text { for all } p, \text { and } \mu(z \mid p)= \begin{cases}0 & \text { for } p<p_{L} \text { and } z \neq z_{L} \\
0 & \text { for } p \neq \tilde{p}(z) \text { and } p \in\left[p_{L}, p_{H}\right] \\
0 & \text { for } p>p_{H} \text { and } z \neq z_{H}\end{cases}
\end{gathered}
$$

The policy is given by:

$$
t_{0}=0, t(p)= \begin{cases}h\left(z_{L}\right)-p & \text { for all } p<p_{L} \\ h\left(\tilde{p}^{-1}(p)\right)-p-\frac{k}{q(\Theta(p))} & \text { and } p \in\left[p_{L}, p_{H}\right] . \\ h\left(z_{H}\right)-p & \text { for } p>p_{H}\end{cases}
$$

\footnotetext{
${ }^{34}$ According to Lemma $7, \tilde{p}(z)$ is strictly increasing in $z$. Also, $\tilde{p}(z)$ is continuous; therefore, the set of prices in the constructed implementable mechanism is $\mathcal{P} \equiv\left[p_{L}, p_{H}\right]$.
} 
The construction is straightforward. We allocate all types the same market tightness and transfer that they were given in the direct mechanism. For construction of off-the-equilibriumpath beliefs, if $p<p_{L}$, then the only type attracted to this post is $z_{L}$. Therefore, $\mu(z \mid p)=0$ for all $z \neq z_{L}$, and $\mu(z \mid p)$ has a mass point at $z=z_{L}$. Similarly, if $p>p_{H}$, then the only type attracted to this price is $z_{H}$. Therefore, $\mu(z \mid p)=0$ for all $z \neq z_{H}$. Given the above beliefs, the tax amount for all $p$ is constructed such that buyers receive a net profit of exactly 0 for $p \in \mathcal{P}$ and $-k$ for $p \notin \mathcal{P}$. Note that the choice of $t$ is not unique for $p \notin \mathcal{P}$. We could construct $t$ differently such that buyers receive any non-positive amount of profit for $p \notin \mathcal{P}$. $G(p)$ is easily constructed given the construction of $\Theta($.$) .$

The conditions for implementability should be verified now. It is easy to check that the buyers' zero profit condition is satisfied because of the construction of $t$. Feasibility or market clearing condition is satisfied because of the construction of $G$. The budget-balance condition is satisfied because of the choice of $U\left(z_{H}\right)$.

Regarding the sellers' optimal search condition, first note that the restriction on off-theequilibrium-path beliefs is equivalent to: ${ }^{35}$

$$
m(\Theta(p))=\min \left\{\bar{m}, i n f_{z \in\{z \mid c(z)<p\}} \frac{U(z)}{p-c(z)}\right\},
$$

if $\{z \mid c(z)<p\}$ is non-empty. Otherwise, set $\Theta(p)=\infty$. Now it is easy to see that sellers' optimal search is satisfied because of the construction of $\Theta(p)$. The only thing worth explaining here is why only $z_{L}$ is attracted to any price less than $p_{L}$ (and similarly, why only $z_{H}$ is attracted to any price greater than $p_{H}$ ). To see why, I begin by writing the incentive compatibility condition for any feasible mechanism: $m\left(\tilde{\theta}\left(z_{L}\right)\right)\left(\tilde{p}\left(z_{L}\right)-c(z)\right) \leq U(z)$ for all $z$. Remember that the payoff to type $z$ is the same in the mechanism and in the proposed allocation. After using the fact that $U\left(z_{L}\right)=m\left(\tilde{\theta}\left(z_{L}\right)\right)\left(\tilde{p}\left(z_{L}\right)-c\left(z_{L}\right)\right)$, one can write:

$$
\begin{gathered}
U\left(z_{L}\right)-U(z) \leq m\left(\tilde{\theta}\left(z_{L}\right)\right)\left(c(z)-c\left(z_{L}\right)\right) \text { for all } z . \\
\Rightarrow U\left(z_{L}\right)-U(z) \leq m\left(\tilde{\theta}\left(z_{L}\right)\right)\left(c(z)-c\left(z_{L}\right)\right)=\frac{U\left(z_{L}\right)}{\tilde{p}\left(z_{L}\right)-c\left(z_{L}\right)}\left(c(z)-c\left(z_{L}\right)\right) \\
\leq \frac{U\left(z_{L}\right)}{p-c\left(z_{L}\right)}\left(c(z)-c\left(z_{L}\right)\right) \text { for all } z \text { and for } p \in\left(c\left(z_{L}\right), p\left(z_{L}\right)\right),
\end{gathered}
$$

or equivalently,

$$
\frac{U\left(z_{L}\right)}{p-c\left(z_{L}\right)} \leq \frac{U(z)}{p-c(z)} \text { for all } z \text { and for } p \in\left(c\left(z_{L}\right), p\left(z_{L}\right)\right) .
$$

Therefore, for the above choice of $m(\Theta(p))$, the restriction on off-the-equilibrium-path beliefs is satisfied.

\footnotetext{
${ }^{35}$ See Chang (2014) for a more detailed discussion.
} 


\section{Proof of Proposition 3: Preliminaries}

Proposition 3 is proved using a guess-and-verify approach. I guess that the first best is achievable, and then I verify the conditions for feasibility. One problem is that if the first best is not achievable, then this approach does not work. To be able to use my characterization method for a general case, I first characterize the incentive compatible schemes, as is common in the mechanism design literature. Next, I work with a modified problem in which the incentive compatibility constraint of sellers has been replaced by other constraints, monotonicity and envelope condition.

Similar to the discrete type space, the budget-balance condition must be satisfied with equality at the optimal mechanism. Furthermore, $\tilde{t}(z)$ can be substituted from part (iii) of Definition 7 in the budget-balance condition. The planner's problem turns into:

\section{Problem 5.}

$$
\begin{gathered}
\max _{\tilde{\theta}(z), \tilde{p}(z)} \int[m(\tilde{\theta}(z))(h(z)-c(z))-k \tilde{\theta}(z)] d F(z) \\
\text { subject to: } z \in \arg \max _{\hat{z}} U(z, \hat{z})(I C), U(z) \geq 0(P C), \\
\int\left[m(\tilde{\theta}(z))(h(z)-\tilde{p}(z))-k \tilde{\theta}(z)-\tilde{t}_{0}\right] d F(z)=0(B B) .
\end{gathered}
$$

Note that no transfer appears in the objective function, because it has been assumed that all types participate in the mechanism. Also, $\int[m(\tilde{\theta}(z)) \tilde{t}(z)] d F(z)$ has been replaced by $\int[m(\tilde{\theta}(z)) h(z)-k \tilde{\theta}(z)] d F(z)$ from the budget-balance condition.

Lemma 6 (Necessary and sufficient condition for incentive compatibility). Assume $c^{\prime}()>$.0 .

(i) Take any mechanism $\{(\tilde{\theta}(z), ., .,)$.$\} that satisfies IC. If \tilde{\theta}(z)$ is a piecewise $C^{1}$ function, then $\frac{d \tilde{\theta}(z)}{d z} \leq 0$ wherever $\tilde{\theta}(z)$ is differentiable at $z$.

(ii) Consider any piecewise $C^{1}$ function $\tilde{\theta}(z)$ satisfying $\frac{d \tilde{\theta}(z)}{d z} \leq 0$. Then there exists transfer schedules $\tilde{p}($.$) such that the mechanism \{(\tilde{\theta}(z), \tilde{p}(),. .,)$.$\} satisfies I C$.

(iii) If mechanism $\{(\tilde{\theta}(z), ., .,)$.$\} satisfies I C$, then $U(z)=U\left(z_{H}\right)+\int_{z}^{z_{H}} m\left(\tilde{\theta}\left(z_{0}\right)\right) c^{\prime}\left(z_{0}\right) d z_{0}$.

Proof. Define $V(W, R, z) \equiv W c(z)+R, w(z) \equiv-m(\theta(z))$ and $r(z) \equiv m(\tilde{\theta}(z)) \tilde{p}(z)+\tilde{t}_{0}$. Obviously, $U(z, \hat{z})=V(w(\hat{z}), r(\hat{z}), z)$. A necessary condition for $w($.$) to satisfy IC is$ $\frac{\partial}{\partial z}\left(\frac{\frac{\partial V}{\partial V}}{\partial R}\right) \frac{d w}{d z} \geq 0$, whenever $w($.$) is differentiable at z$, according to Theorem 7.1, Fudenberg and Tirole (1991). But $\frac{\partial}{\partial z}\left(\frac{\frac{\partial V}{\partial W}}{\frac{\partial V}{\partial R}}\right) \frac{d w}{d z}=\frac{\partial}{\partial z}\left(\frac{c(z)}{1}\right)\left(-m^{\prime}(\tilde{\theta}(z))\right) \frac{d \tilde{\theta}(z)}{d z}$. Also $c^{\prime}()>$.0 and $m^{\prime}() \geq$.0 ; therefore, the necessary condition is equivalent to

$$
c^{\prime}(z) \frac{d \tilde{\theta}(z)}{d z} \leq 0
$$

According to Theorem 7.3 in Fudenberg and Tirole (1991), a sufficient condition for $w($. to satisfy IC is that $\frac{d w(z)}{d z} \geq 0$, or equivalently, $c^{\prime}(z) \frac{d \tilde{\theta}(z)}{d z} \leq 0$. 
For the third part of the lemma, I use Corollary 1 in Milgrom and Segal (2002). Their result states that if $\tilde{\theta}(z)$ satisfies IC, then $U($.$) can be written as follows:$

$$
U(z)=U\left(z_{H}\right)-\int_{z}^{z_{H}} \frac{\partial U\left(z_{0}, z_{0}\right)}{\partial z} d z_{0}=U\left(z_{H}\right)+\int_{z}^{z_{H}} m\left(\tilde{\theta}\left(z_{0}\right)\right) c^{\prime}\left(z_{0}\right) d z_{0} .
$$

This equation is derived from the envelope theorem and is standard in the mechanism design literature. The requirements of the result of Milgrom and Segal (2002) are as follows:

1. $U(z, \hat{z})$ is differentiable and absolutely continuous in $z$. This is satisfied because $c$ is assumed to be twice differentiable.

2. $\sup _{\hat{z}}\left|\frac{\partial U(z, \hat{z})}{\partial z}\right|$ is integrable. This is satisfied because $\sup _{\hat{z}}\left|\frac{\partial U(z, \hat{z})}{\partial z}\right| \leq\left|c^{\prime}(z)\right|<M$ for some $M \in \mathbb{R}_{+}$, because $c^{\prime}($.$) is continuous and is defined over a compact set \left[z_{L}, z_{H}\right]$.

3. $\tilde{\theta}(z)$ is obviously non-empty.

From IC, $U(z)=m(\tilde{\theta}(z))(\tilde{p}(z)-c(z))+\tilde{t}_{0}$ for all $z$. From now on it is assumed that $\tilde{t}_{0}=0$, which is without loss of generality if all types are active, i.e., $\tilde{\theta}(z)>0$, because then $\tilde{p}(z)$ can be changed to $\tilde{p}(z)+\frac{\tilde{t}_{0}}{m(\tilde{\theta}(z))}$. It can be easily verified that all types should be active under requirements of Proposition 3. I substitute $U($.$) from (30) into U(z)=m(\tilde{\theta}(z))(\tilde{p}(z)-c(z))$ to derive transfers:

$$
\tilde{p}(z)=c(z)+\frac{U\left(z_{H}\right)+\int_{z}^{z_{H}} m\left(\tilde{\theta}\left(z_{0}\right)\right) c^{\prime}\left(z_{0}\right) d z_{0}}{m(\tilde{\theta}(z))} .
$$

Now, budget-balance condition is used to derive $U\left(z_{H}\right)$ :

$$
\begin{gathered}
0=\int[m(\tilde{\theta}(z))[h(z)-\tilde{p}(z)]-k \tilde{\theta}(z)] F^{\prime}(z) d z \\
=\int[m(\tilde{\theta}(z))[h(z)-c(z)]-k \tilde{\theta}(z)-m(\tilde{\theta}(z))(\tilde{p}(z)-c(z))] F^{\prime}(z) d z \\
=\int\left[m(\tilde{\theta}(z))(h(z)-c(z))-k \tilde{\theta}(z)-\int_{z}^{z_{H}} m\left(\tilde{\theta}\left(z_{0}\right)\right) c^{\prime}\left(z_{0}\right) d z_{0}-U\left(z_{H}\right)\right] F^{\prime}(z) d z .
\end{gathered}
$$

The second equality follows from (31). The third equality uses the relationship between $U(z)$ and $\tilde{p}(z)$ and also (30). Using integration by parts, one yields: ${ }^{36}$

$$
U\left(z_{H}\right)=\int\left[m(\tilde{\theta}(z))\left(h(z)-c(z)-c^{\prime}(z) \frac{F(z)}{F^{\prime}(z)}\right)-k \tilde{\theta}(z)\right] F^{\prime}(z) d z
$$

According to (30) and because $c^{\prime}()>$.0 , if $U\left(z_{H}\right) \geq 0$, then $U(z) \geq 0$ for all $z$. Hence, $U\left(z_{H}\right) \geq 0$ implies that the planner's budget-balance condition and participation constraint of all types are satisfied. The IC constraint in the planner's problem has been reduced to (29) and (31). The planner's budget-balance condition and participation constraint of all types are also summarized in $U\left(z_{H}\right) \geq 0$. Therefore, thanks to Lemma 6 , the planner's problem can be written as follows to derive $\tilde{\theta}(z)$, and $\tilde{p}(z)$ will be automatically given by (31).

\footnotetext{
${ }^{36}$ For any differentiable functions $F$ and $G$, if $G\left(z_{H}\right)=0$, and $F\left(z_{L}\right)=0$ one will have: $\int_{z_{L}}^{z_{H}} F^{\prime}(z) G(z) d z=$ $-\int_{z}^{z_{H}} F(z) G^{\prime}(z) d z$ using integration by parts. In the above equality, set $G(z)=\int_{z}^{z_{H}} m\left(\tilde{\theta}\left(z_{0}\right)\right) c^{\prime}\left(z_{0}\right) d z_{0}$.
} 


\section{Problem 6.}

$$
\begin{gathered}
\max _{\tilde{\theta}(z)} \int[m(\tilde{\theta}(z))[h(z)-c(z)]-k \tilde{\theta}(z)] F^{\prime}(z) d z \\
\text { subject to } c^{\prime}(z) \frac{d \tilde{\theta}(z)}{d z} \leq 0(\text { monotonicity constraint; } M C), \\
U\left(z_{H}\right) \equiv \int\left[m(\tilde{\theta}(z))\left(h(z)-c(z)-c^{\prime}(z) \frac{F(z)}{F^{\prime}(z)}\right)-k \tilde{\theta}(z)\right] F^{\prime}(z) d z \geq 0 .
\end{gathered}
$$

For all parts of Proposition 3, the following direct mechanism is proposed:

$$
\begin{gathered}
\tilde{\theta}^{*}(z)=\theta^{F B}(z), \\
\tilde{p}^{*}(z)=c(z)+\frac{U^{*}\left(z_{H}\right)+\int_{z}^{z_{H}} m\left(\tilde{\theta}^{*}\left(z_{0}\right)\right) c^{\prime}\left(z_{0}\right) d z_{0}}{m\left(\theta^{F B}(z)\right)},
\end{gathered}
$$

where $U^{*}\left(z_{H}\right)=\int\left[m\left(\tilde{\theta}^{*}(z)\right)(h(z)-c(z))-m\left(\tilde{\theta}^{*}(z)\right) c^{\prime}(z) \frac{F(z)}{F^{\prime}(z)}\right] F^{\prime}(z) d z$,

$$
\tilde{t}_{0}^{*}=0 \text { and } \tilde{t}^{*}(z)=h(z)-\tilde{p}^{*}(z)-\frac{k}{q\left(\tilde{\theta}^{*}(z)\right)} \text { for all } z \text {. }
$$

Proof of Proposition 3(i). I need to check that the two constraints of Problem 6 are satisfied for the proposed mechanism under the respective assumptions. The first best level of market tightness, $\theta^{F B}(z)$, is given by $m^{\prime}\left(\theta^{F B}(z)\right)(h(z)-c(z))-k=0$. By differentiating it with respect to $z$, one yields $\frac{d \theta^{F B}(z)}{d z}=-\frac{k\left(h^{\prime}(z)-c^{\prime}(z)\right)}{m^{\prime \prime}\left(\theta^{F B}(z)\right)(h(z)-c(z))^{2}} \leq 0$, where the inequality is due to the fact that $h^{\prime}()-.c^{\prime}() \leq$.0 and $m^{\prime \prime}() \leq$.0 . Hence, MC in problem 6 is satisfied. Moreover, $U^{*}\left(z_{H}\right)=\int\left[m(\tilde{\theta}(z))\left(h(z)-c(z)-c^{\prime}(z) \frac{F(z)}{F^{\prime}(z)}\right)-k \tilde{\theta}(z)\right] F^{\prime}(z) d z$

$$
\begin{gathered}
=\int\left[\left(-\int_{z}^{z_{H}} m\left(\theta^{F B}(z)\right)\left(h^{\prime}(z)-c^{\prime}(z)\right) d z+U^{F B}\left(z_{H}\right)\right)-m\left(\theta^{F B}(z)\right) c^{\prime}(z) \frac{F(z)}{F^{\prime}(z)}\right] F^{\prime}(z) d z \\
=-\int\left[m\left(\theta^{F B}(z)\right)\left(h^{\prime}(z)-c^{\prime}(z)\right)+m\left(\theta^{F B}(z)\right) c^{\prime}(z)\right] F(z) d z+U^{F B}\left(z_{H}\right) \\
=-\int m\left(\theta^{F B}(z)\right) h^{\prime}(z) F(z) d z+U^{F B}\left(z_{H}\right) \geq 0 .
\end{gathered}
$$

The second equality uses the fact that $\tilde{\theta}(z)=\theta^{F B}(z)$ and also the fact that $\frac{d U^{F B}(z)}{d z}=$ $\frac{d\left[\max _{\theta}\{m(\theta)(h(z)-c(z))-k \theta\}\right]}{d z}=m\left(\theta^{F B}\right)\left(h^{\prime}(z)-c^{\prime}(z)\right)$. The third equality is derived by using integration by parts. The inequality holds because $h^{\prime}(z)<0$ by assumption, and $U^{F B}\left(z_{H}\right) \geq 0$ because there are positive gains from trade for all types. Both constraints in Problem 6 are satisfied. Finally, the proposed mechanism achieves the first best, which is the highest possible welfare, so it is not needed to check whether any other allocation achieves higher welfare. $^{37}$

\footnotetext{
${ }^{37}$ The proposition and its proof can be written in the same fashion if $c($.$) is strictly decreasing, instead.$ To save space, these results are not reported.
} 
Proof of Proposition 3(ii). By part (i), the planner achieves the first best. By IC, $U^{\prime *}(z)=-m\left(\theta^{F B}(z)\right) c^{\prime}(z)$, and $U^{\prime E Q}(z)=-m\left(\theta^{E Q}(z)\right) c^{\prime}(z)$ almost everywhere. Since $h^{\prime}(z) \leq 0<c^{\prime}(z)$, then the boundary condition in the equilibrium is $\theta^{E Q}\left(z_{H}\right)=\theta^{F B}\left(z_{H}\right)$. Equilibrium condition (14) implies that $\theta^{E Q}(z) \geq \theta^{F B}(z)$ for all $z$. Therefore, $U^{* *}(z) \geq$ $U^{\prime E Q}(z)$ almost everywhere. To show that the equilibrium allocation is Pareto dominated by the allocation implemented by the proposed mechanism, it is necessary and sufficient to show that $U^{*}\left(z_{L}\right) \geq U^{E Q}\left(z_{L}\right)$. But $U^{*}\left(z_{L}\right)=U^{*}\left(z_{H}\right)+\int_{z_{L}}^{z_{H}} m\left(\theta^{F B}(z)\right) c^{\prime}(z) d z=U^{F B}\left(z_{H}\right)-$ $\int_{z_{L}}^{z_{H}} m\left(\theta^{F B}(z)\right) h^{\prime}(z) F(z) d z+\int_{z_{L}}^{z_{H}} m\left(\theta^{F B}(z)\right) c^{\prime}(z) d z$. Moreover,

$$
U^{E Q}\left(z_{L}\right)=U^{E Q}\left(z_{H}\right)+\int_{z_{L}}^{z_{H}} m\left(\theta^{E Q}(z)\right) c^{\prime}(z) d z=U^{F B}\left(z_{H}\right)+\int_{z_{L}}^{z_{H}} m\left(\theta^{E Q}(z)\right) c^{\prime}(z) d z
$$

Hence, it is necessary and sufficient to show that

$$
-\int_{z_{L}}^{z_{H}} m\left(\theta^{F B}(z)\right) h^{\prime}(z) F(z) d z \geq \int\left(m\left(\theta^{E Q}(z)\right)-m\left(\theta^{F B}(z)\right)\right) c^{\prime}(z) d z .
$$

The proof is now complete, because the LHS is exactly the same as the LHS of (6) using integration by parts.

Proof of Proposition 3(iii), "if" part. Again, I need to show that the proposed mechanism is feasible. But $h^{\prime}(z)-c^{\prime}(z) \leq 0$, so $\frac{d \theta^{F B}(z)}{d z} \leq 0$, thus the first constraint in Problem 6 is satisfied. Furthermore,

$$
\begin{gathered}
U^{*}\left(z_{H}\right)=\int\left[m\left(\theta^{F B}(z)\right)[h(z)-c(z)]-k \theta^{F B}(z)-m\left(\theta^{F B}(z)\right) c^{\prime}(z) \frac{F(z)}{F^{\prime}(z)}\right] d F(z) \\
=\int\left[\int_{z_{L}}^{z} m\left(\theta^{F B}(\tilde{z})\right)\left(h^{\prime}(\tilde{z})-c^{\prime}(\tilde{z})\right) d \tilde{z}+U^{F B}\left(z_{L}\right)-m\left(\theta^{F B}(z)\right) c^{\prime}(z) \frac{F(z)}{F^{\prime}(z)}\right] d F(z) \\
=\int H_{2}(z) d F(z)-\iint_{z_{L}}^{z} m\left(\theta^{F B}(\tilde{z})\right) c^{\prime}(\tilde{z}) d \tilde{z} d F(z)-\int m\left(\theta^{F B}(z)\right) c^{\prime}(z) F(z) d z+U^{F B}\left(z_{L}\right) \\
=\int H_{2}(z) d F(z)-\left(\int m\left(\theta^{F B}(z)\right) c^{\prime}(z) d z-U^{F B}\left(z_{L}\right)\right) \geq 0
\end{gathered}
$$

where the last equality is derived using integration by parts and the last inequality is derived using (7). ${ }^{38}$

Proof of Proposition 3(iii), "only if" part. The planner achieves the FB, so $\theta^{F B}(z)$ must solve Problem 6, thus the second constraint, specifically, must hold. Exactly similar to the last part, one can manipulate the integral to show that condition (7) must be satisfied, too.

\footnotetext{
${ }^{38}$ To show that (7) and (11) are equivalent, begin from the integral in the first line above. That is positive if and only if (7) is satisfied. Now notice that the integrand is equal to $m\left(\theta^{F B}(z)\right)[h(z)-c(z)]-k \theta^{F B}(z)-$ $m\left(\theta^{F B}(z)\right) c^{\prime}(z) \frac{F(z)}{F^{\prime}(z)}=-\frac{\theta^{F B}(z) q^{\prime}\left(\theta^{F B}(z)\right)}{q\left(\theta^{F B}(z)\right)}(h(z)-c(z)) m\left(\theta^{F B}(z)\right)-m\left(\theta^{F B}(z)\right) c^{\prime}(z) \frac{F(z)}{F^{\prime}(z)}$, where (4) is used for the equality. But the latter expression is the same integrand as in (11).
} 
Proof of Proposition 3(iv). Since $h^{\prime}(z) \geq 0$, it is easy to see that (8) is stronger than (7); therefore, the planner achieves the first best in this case too, according to part (iii). Similarly as above, by IC we have $U^{\prime *}(z)=-m\left(\theta^{F B}(z)\right) c^{\prime}(z)$ and $U^{\prime E Q}(z)=-m\left(\theta^{E Q}(z)\right) c^{\prime}(z)$ almost everywhere. Since $h^{\prime}(z) \geq 0$, then the boundary condition in the equilibrium is $\theta^{E Q}\left(z_{L}\right)=\theta^{F B}\left(z_{L}\right)$. Equilibrium condition (14) implies that $\theta^{E Q}(z) \leq \theta^{F B}(z)$ for all $z$. Therefore, $U^{\prime *}(z) \leq U^{\prime E Q}(z)$ almost everywhere.

To show that the equilibrium allocation is Pareto dominated by the constrained efficient allocation, it is necessary and sufficient to show that $U^{*}\left(z_{H}\right) \geq U^{E Q}\left(z_{H}\right)$. From (35), one yields $U^{*}\left(z_{H}\right)=U^{F B}\left(z_{H}\right)-\int_{z_{L}}^{z_{H}} m\left(\theta^{F B}(z)\right) h^{\prime}(z) F(z) d z$. Moreover,

$$
\begin{gathered}
U^{E Q}\left(z_{H}\right)=U^{E Q}\left(z_{L}\right)-\int_{z_{L}}^{z_{H}} m\left(\theta^{E Q}(z)\right) c^{\prime}(z) d z=U^{F B}\left(z_{L}\right)-\int_{z_{L}}^{z_{H}} m\left(\theta^{E Q}(z)\right) c^{\prime}(z) d z \\
=U^{F B}\left(z_{H}\right)-\int_{z_{L}}^{z_{H}} m\left(\theta^{F B}(z)\right)\left(h^{\prime}(z)-c^{\prime}(z)\right) d z-\int_{z_{L}}^{z_{H}} m\left(\theta^{E Q}(z)\right) c^{\prime}(z) d z .
\end{gathered}
$$

Hence, it is necessary and sufficient to show that

$$
\int_{z_{L}}^{z_{H}} m\left(\theta^{F B}(z)\right) h^{\prime}(z)(1-F(z)) d z \geq \int\left(m\left(\theta^{F B}(z)\right)-m\left(\theta^{E Q}(z)\right)\right) c^{\prime}(z) d z .
$$

The proof is now complete, because the LHS is exactly the same as the LHS of (8) after integrating by parts.

Proof of Lemma 2. Again, I suppress the superscript $*$ in this proof to reduce the notation when there is no danger of confusion. Proposition 3(iii) applies, so $\tilde{\theta}(z)=\theta^{F B}(z)$ for all $z$. I calculate $m(\tilde{\theta}(z)) \tilde{t}(z)$ and take its derivative with respect to $z$ :

$$
\begin{gathered}
m(\tilde{\theta}(z)) \tilde{t}(z)=m\left(\theta^{F B}(z)\right)(h(z)-c(z))-k \theta^{F B}(z)-U\left(z_{H}\right)-\int_{z}^{z_{H}} m\left(\theta^{F B}\left(z_{0}\right)\right) c^{\prime}\left(z_{0}\right) d z_{0} \\
=U^{F B}(z)-U\left(z_{H}\right)-\int_{z}^{z_{H}} m\left(\theta^{F B}\left(z_{0}\right)\right) c^{\prime}\left(z_{0}\right) d z_{0} \\
\Rightarrow \frac{\partial}{\partial z}[m(\tilde{\theta}(z)) \tilde{t}(z)]=\frac{d U^{F B}(z)}{\partial z}+m\left(\theta^{F B}(z)\right) c^{\prime}(z)=m\left(\theta^{F B}(z)\right) h^{\prime}(z) \geq 0
\end{gathered}
$$

The second equality is derived by applying envelope theorem to the following maximization problem: $U^{F B}(z)=\max _{\theta}\{m(\theta)(h(z)-c(z))-k \theta\}$. The inequality holds by assumption.

Since $\theta^{F B}($.$) is strictly decreasing, the associated implementable allocation must be sep-$ arating. I show below that $\tilde{t}(z)$ is decreasing in $z$ at $z=z_{L}$. Furthermore, $\frac{d t}{d p}=\frac{d \tilde{t} / d z}{d \tilde{p} / d z}$, and as shown in Lemma 7 that the denominator is always positive, $\frac{d t}{d p}$ must be negative for $p=p_{L}$. Notice that Lemma 7 holds with strict monotonicity for almost all $z$ because allocation is separating. According to (36), $\frac{d[m(\tilde{\theta}(z)) \tilde{t}(z)]}{d z}=m(\tilde{\theta}(z)) h^{\prime}(z)$; therefore,

$$
\frac{d \tilde{t}}{d z}=h^{\prime}(z)-\frac{m^{\prime}(\tilde{\theta}(z))}{m(\tilde{\theta}(z))} \frac{d \tilde{\theta}(z)}{d z} \tilde{t}(z) .
$$


Also, $\frac{d \tilde{\theta}(z)}{d z}$ can be calculated from (4) to obtain $\frac{d \tilde{t}}{d z}$ as in Lemma 2. Consider this equality for $z=z_{L}$. Given the assumption that $h^{\prime}\left(z_{L}\right)=0$ and given the fact that $\tilde{\theta}^{\prime}(z)<0$ for all $z$, it is sufficient to show that $\tilde{t}\left(z_{L}\right)<0$. It will follow that $\tilde{t}^{\prime}\left(z_{L}\right)<0$. To calculate $\tilde{t}(z)$, I use the planner's budget-balance condition to write: $\int m(\tilde{\theta}(z)) \tilde{t}(z) d F(z)=0$. Let $\chi(.) \equiv m(\tilde{\theta}(z)) \tilde{t}(z)$, then $0=\int \chi(z) d F(z)=-\left.\chi(z)(1-F(z))\right|_{z_{L}} ^{z_{H}}+\int \chi^{\prime}(z)(1-F(z)) d z$ by using integration by parts. Therefore, $\chi\left(z_{L}\right)=-\int \chi^{\prime}(z)(1-F(z)) d z<0$. The inequality holds because $\chi^{\prime}(z)=m(\tilde{\theta}(z)) h^{\prime}(z)$ from (36) and the fact that $h^{\prime}(z) \geq 0$. Also, the inequality is strict because $h^{\prime}(z)>0$ for a positive measure of $z$. But $\tilde{t}\left(z_{L}\right)=\frac{\chi\left(z_{L}\right)}{m\left(\tilde{\theta}\left(z_{L}\right)\right)}<0$ by definition of $\chi($.$) .$

Finally, I show that $\frac{d t}{d p}>0$ for some $p$. The facts that $\int m(\tilde{\theta}(z)) \tilde{t}(z) d F(z)=0, \tilde{t}\left(z_{L}\right)<0$, $\tilde{t}(z)$ is continuous, and $F$ has full support, together imply that $\tilde{t}(z)$ must be strictly positive for a strictly positive measure of $z$, therefore, $\tilde{t}^{\prime}(z)>0$ for for a strictly positive measure of $z$. Finally, $\frac{d t}{d p}=\frac{d \tilde{t} / d z}{d \tilde{p} / d z}$. Again, according to Lemma $7, \frac{d t}{d p}$ must be strictly positive for some $p$.

Lemma 7. Assume $c^{\prime}()>$.0 . Take any feasible mechanism in which all types receive a strictly positive payoff, and in which the market tightness allocated to different types is all different. Price function in this mechanism, $\tilde{p}(z)$, is strictly increasing for almost all z.

Proof of Lemma 7. According to (9), $\frac{d[m(\tilde{\theta}(z)) \tilde{p}(z)]}{d z}=m^{\prime}(\tilde{\theta}(z)) \frac{d \tilde{\theta}(z)}{d z} c(z)$, so

$$
\frac{d \tilde{p}(z)}{d z}=-\frac{m^{\prime}(\tilde{\theta}(z))}{m(\tilde{\theta}(z))} \frac{d \tilde{\theta}(z)}{d z}(\tilde{p}(z)-c(z)) \geq 0 .
$$

The inequality holds because $\tilde{\theta}(z)$ is decreasing in $z$ following the fact that the mechanism satisfies IC. Moreover, $\tilde{p}(z)-c(z)$ is positive for all types following the fact that the mechanism satisfies the participation constraint. Moreover, the inequality is strict for almost all types as $\tilde{p}(z)-c(z)=\frac{U(z)}{m(\tilde{\theta}(z))}=\frac{U\left(z_{H}\right)+\int_{z}^{z_{H}} m\left(\tilde{\theta}\left(z_{0}\right)\right) c^{\prime}\left(z_{0}\right) d z_{0}}{m(\tilde{\theta}(z))}>\frac{U\left(z_{H}\right)}{m(\tilde{\theta}(z))} \geq 0$ for $z<z_{H}$.

Proof of Proposition 5. This proof is similar to the proof of Lemma 5. One difference is that here the set of admissible prices, $\mathbb{P}$, is assumed to be $\left[c\left(z_{L}\right), \infty\right)$ as opposed to $(0, \infty)$. This assumption is not restrictive (and made only to avoid some technical difficulties), as no seller would have incentives to apply to $p<c\left(z_{L}\right)$.

Consider again a feasible mechanism $\left\{\tilde{\theta}(),. \tilde{p}(),. \tilde{t}(),. \tilde{t}_{0}\right\}$. I construct the allocation $\{G, \mathcal{P}, \Theta, \mu\}$ and policy $\left\{t, t_{e}, t_{0}\right\}$ and show that if $M \in \mathbb{R}_{+}$and $M^{\prime} \in \mathbb{R}_{+}$, defined below, are chosen sufficiently large, then this allocation is implementable and $t_{e}(p)$ is strictly decreasing and $t(p)$ is strictly increasing in $p$. The allocation is constructed as follows:

$$
\mathcal{P} \equiv\left[p_{L}, p_{H}\right] \subseteq \mathbb{P} \equiv R_{+} \text {where } p_{L} \equiv \tilde{p}\left(z_{L}\right) \text { and } p_{H} \equiv \tilde{p}\left(z_{H}\right)
$$


and $\tilde{p}^{*}$ is given by (9) (where $\tilde{\theta}$ should replace $\tilde{\theta}^{*}$ ). Moreover,

$$
\begin{gathered}
\begin{cases}\Theta(p)=1 & \text { for } p=c\left(z_{L}\right) \\
m(\Theta(p))=\min \left\{\bar{m}, \frac{U\left(z_{L}\right)}{p-c\left(z_{L}\right)}\right\} & \text { for } p \in\left(c\left(z_{L}\right), p_{L}\right) \\
\Theta(p)=\tilde{\theta}\left(\tilde{p}^{-1}(p)\right) & \text { for } p \in\left[p_{L}, p_{H}\right] \\
m(\Theta(p))=\min \left\{\bar{m}, \frac{U\left(z_{H}\right)}{p-c\left(z_{H}\right)}\right\} & \text { for } p>p_{H}\end{cases} \\
G(p)= \begin{cases}0 & \text { for } p \in\left[c\left(z_{L}\right), p_{L}\right) \\
\int_{p_{L}}^{p} \Theta(p) F^{\prime}\left(\tilde{p}^{-1}(p)\right) d p & \text { for } p \in\left[p_{L}, p_{H}\right] \\
1 & \text { for } p>p_{H}\end{cases} \\
\int \mu(z \mid p) d z=1 \text { for all } p, \text { and } \mu(z \mid p)= \begin{cases}0 & \text { for } p<p_{L} \text { and } z \neq z_{L} \\
0 & \text { for } p \neq \tilde{p}(z) \text { and } p \in\left[p_{L}, p_{H}\right] \\
0 & \text { for } p>p_{H} \text { and } z \neq z_{H}\end{cases}
\end{gathered}
$$

The policy is given by:

$$
\begin{gathered}
\tilde{t}_{0}=0, t_{e}(p)= \begin{cases}-k+M\left(p_{H}-p\right) & \text { for } p \in\left[c\left(z_{L}\right), p_{H}\right] \\
-k & \text { for } p>p_{H}\end{cases} \\
t(p)= \begin{cases}h\left(z_{L}\right)-p-\frac{k+t_{e}(p)}{q(\Theta(p))} & \text { for all } p \in\left[c\left(z_{L}\right), p_{L}\right) \\
h\left(\tilde{p}^{-1}(p)\right)-p-\frac{k+t_{e}(p)}{q(\Theta(p))} & \text { and } p \in\left[p_{L}, p_{H}\right] \\
t\left(p_{H}\right)+M^{\prime}\left(p-p_{H}\right) & \text { for } p>p_{H}\end{cases}
\end{gathered}
$$

The conditions for implementability can be verified in a similar fashion as in the proof of Lemma 5(i), so I do not repeat them here.

Regarding monotonicity of taxes, it is obvious that $t_{e}(p)$ is decreasing in $p$ for all $p \in$ $\left[p_{L}, p_{H}\right]$ if $M>0$. It is just left to show that $t(p)$ is increasing in $p$ for $p \in\left[p_{L}, p_{H}\right]$. I take a derivative of $t(p)$ with respect to $p$ :

$$
t^{\prime}(p)=h^{\prime}\left(\tilde{p}^{-1}(p)\right) \frac{d\left(\tilde{p}^{-1}(p)\right)}{d p}-1+M \frac{q(\Theta(p))+q^{\prime}(\Theta(p)) \Theta^{\prime}(p)\left(p_{H}-p\right)}{q(\Theta(p))^{2}} .
$$

Now, define

$M_{1} \equiv \max \left\{1, \sup _{p \in\left[p_{L}, p_{H}\right]} \frac{\left(1-h^{\prime}\left(\tilde{p}^{-1}(p)\right) \frac{d\left(\tilde{p}^{-1}(p)\right)}{d p}\right) q(\Theta(p))^{2}}{q(\Theta(p))+q^{\prime}(\Theta(p)) \Theta^{\prime}(p)\left(p_{H}-p\right)}, \sup _{p \in\left[c\left(z_{L}\right), p_{L}\right]} \frac{q(\Theta(p))^{2}}{q(\Theta(p))+q^{\prime}(\Theta(p)) \Theta^{\prime}(p)\left(p_{H}-p\right)}\right\}$.

$M_{1}$ is a lower bound for $M$ and 1 is merely an arbitrary positive number. I want to show that $M_{1}<\infty$, so the second and third expressions in the max have to be less than $\infty$. Consider the second one. If $q(\Theta(p)) \rightarrow 0$, then the expression goes to 0 ; therefore, I simply need to show that $\frac{d\left(\tilde{p}^{-1}(p)\right)}{d p}>-\infty$. But $\frac{d \tilde{p}}{d z}$ has been already calculated in Lemma 7 , so $\frac{d\left(\tilde{p}^{-1}(p)\right)}{d p}$, which is merely the inverse of $\frac{d \tilde{p}}{d z}$, is always positive too. Since $z$ lies in a compact interval, 
$1-h^{\prime}(.) \frac{d \tilde{p}}{d z}$ is not greater than 1 and the proof in this part is complete. The same argument applies to the third expression but for $p \in\left[c\left(z_{L}\right), p_{L}\right]$.

Therefore, if $M>M_{1}$ and $M^{\prime}>0$, then $t(p)$ is strictly increasing over each separate interval. Since $t(p)$ is continuous by construction, it is therefore increasing over the entire domain.

Example 3 (Characterizing equilibrium and constrained efficient allocation for $m(\theta)=\mu \min \{\theta, 1\}$ ). Assume $0<h^{\prime}(z) \leq c^{\prime}(z)$ and $\mu(h(z)-c(z))>k$ for all $z$. According to differential equation (14), $\tilde{\theta}^{E Q}(z)$ solves:

$$
\tilde{\theta}^{E Q}(z) \exp \left(\int_{z_{L}}^{z} \frac{q\left(\tilde{\theta}^{E Q}(\hat{z})\right) h^{\prime}(\hat{z})}{m^{\prime}\left(\tilde{\theta}^{E Q}(\hat{z})\right)(h(\hat{z})-c(\hat{z}))-k} d \hat{z}\right)=\text { const. }
$$

But $0<h^{\prime}(.) \leq c^{\prime}($.$) , so \tilde{\theta}^{E Q}\left(z_{L}\right)=\theta^{F B}\left(z_{L}\right)$, thus const $=\theta^{F B}\left(z_{L}\right)$. The equilibrium market tightness is distorted downward for all $z>z_{L}$, so $q($.$) and m^{\prime}($.$) , given by q(\theta)=m^{\prime}(\theta)=\mu$ for all $\theta<1$, are both differentiable almost everywhere. Therefore,

$$
\tilde{\theta}^{E Q}(z)=\theta^{F B}\left(z_{L}\right) \exp \left(-\int_{z_{L}}^{z} \frac{h^{\prime}(\hat{z})}{h(\hat{z})-c(\hat{z})-k / \mu} d \hat{z}\right)
$$

Moreover, $\theta^{F B}(z)=1$ for all $z$, so $H_{2}=h(z)-h\left(z_{L}\right)$ and $\bar{H}_{2}=\int h(z) d F(z)-h\left(z_{L}\right) \equiv$ $\mathbb{E}(h(z))-h\left(z_{L}\right)$. Therefore, (8) in this example reduces to:

$$
\mathbb{E}(h(z))-h\left(z_{L}\right) \geq \int\left(1-\exp \left(-\int_{z_{L}}^{z} \frac{h^{\prime}(\hat{z})}{h(\hat{z})-c(\hat{z})-k / \mu} d \hat{z}\right)\right) c^{\prime}(\hat{z}) d z .
$$

This condition is explicitly on the fundamentals of the model. Furthermore, (6) can be given in a similar fashion.

With this special matching function, however, $\eta(\theta)=0$ for $\theta<1$, so condition (11), which is equivalent to (7), is not satisfied. Hence, the planner cannot achieve the first best. Notice that condition (8) is not satisfied either, because it is even stronger than (7), but I derived it explicitly here to show what it looks like. 


\section{References}

D. Acemoglu and R. Shimer. Holdups and efficiency with search frictions. International Economic Review, 40(4):827-849, 1999a.

D. Acemoglu and R. Shimer. Efficient unemployment insurance. Journal of Political Economy, 107(5): 893-928, 1999b.

G. Akerlof. The economics of caste and of the rat race and other woeful tales. The Quarterly Journal of Economics, 90(4):599-617, 1976.

G. A. Akerlof. The market for "lemons": Quality uncertainty and the market mechanism. The Quarterly Journal of Economics, 84(3):488-500, 1970.

C. D. Aliprantis and K. C. Border. Infinite Dimensional Analysis: A Hitchhikers Guide. New York: SpringerVerlag, 1986.

D. Balkenborg and M. Makris. An undominated mechanism for a class of informed principal problems with common values. Journal of Economic Theory, 157:918-958, 2015.

M. Cella. Informed principal with correlation. Games and Economic Behavior, 64(2):433-456, 2008.

B. Chang. Adverse selection and liquidity distortion. Available at https:/uwmadison.app.box.com/s/ z75iwedb78nmy9dipvmt, 2014.

J. Chiu and T. V. Koeppl. Trading dynamics with adverse selection and search: Market freeze, intervention and recovery. The Review of Economic Studies, 83(3):969-1000, 2016.

I.-K. Cho and D. M. Kreps. Signaling games and stable equilibria. The Quarterly Journal of Economics, 102(2):179-221, 1987.

S. M. Davoodalhosseini. Directed search with complementarity and adverse selection. Available at SSRN: https://ssrn.com/abstract=2925531, 2015.

A. Delacroix and S. Shi. Pricing and signaling with frictions. Journal of Economic Theory, 148(4):1301-1332, 2013.

P. Dubey and J. Geanakoplos. Competitive pooling: Rothschild-stiglitz reconsidered. The Quarterly Journal of Economics, 117(4):1529-1570, 2002.

P. Dubey, J. Geanakoplos, and M. Shubik. Default and punishment in general equilibrium. Econometrica: Journal of the Econometric Society, 73(1):1-37, 2005.

J. Eeckhout and P. Kircher. Sorting and decentralized price competition. Econometrica: Journal of the Econometric Society, 78(2):539-574, 2010.

D. Fudenberg and J. Tirole. Game Theory. Cambridge, MA: The MIT Press, 1991.

D. Gale. A Walrasian theory of markets with adverse selection. The Review of Economic Studies, 59(2): 229-255, 1992.

D. Gale. Equilibria and Pareto optima of markets with adverse selection. Economic Theory, 7(2):207-235, 1996.

M. Galenianos and P. Kircher. Directed search with multiple job applications. Journal of Economic Theory, 144(2):445-471, 2009. 
M. Golosov, P. Maziero, and G. Menzio. Taxation and redistribution of residual income inequality. Journal of Political Economy, 121(6):1160-1204, 2013.

B. C. Greenwald and J. E. Stiglitz. Externalities in economies with imperfect information and incomplete markets. The Quarterly Journal of Economics, 101(2):229-264, 1986.

V. Guerrieri. Inefficient unemployment dynamics under asymmetric information. Journal of Political Economy, 116(4):667-708, 2008.

V. Guerrieri and R. Shimer. Dynamic adverse selection: A theory of illiquidity, fire sales, and flight to quality. American Economic Review, 104(7):1875-1908, 2014a.

V. Guerrieri and R. Shimer. Markets with multidimensional private information. Technical report, National Bureau of Economic Research, 2014b.

V. Guerrieri, R. Shimer, and R. Wright. Adverse selection in competitive search equilibrium. Econometrica: Journal of the Econometric Society, 78(6):1823-1862, 2010.

B. Holmström and R. B. Myerson. Efficient and durable decision rules with incomplete information. Econometrica: Journal of the Econometric Society, 51(6):1799-1819, 1983.

R. Inderst and A. Wambach. Competitive insurance markets under adverse selection and capacity constraints. European Economic Review, 45(10):1981-1992, 2001.

R. Inderst and A. Wambach. Capacity constrained firms in (labor) markets with adverse selection. Economic Theory, 19(3):525-548, 2002.

B. Jullien. Participation constraints in adverse selection models. Journal of Economic Theory, 93(1):1-47, 2000 .

J.-J. Laffont and D. Martimort. The theory of incentives: the principal-agent model. Princeton University Press, 2009.

B. Lester, A. Shourideh, V. Venkateswaran, and A. Zetlin-Jones. Screening and adverse selection in frictional markets. Technical report, National Bureau of Economic Research, 2015.

E. Maskin and J. Tirole. The principal-agent relationship with an informed principal, ii: Common values. Econometrica: Journal of the Econometric Society, 60(1):1-42, 1992.

G. Menzio and S. Shi. Efficient search on the job and the business cycle. Journal of Political Economy, 119 (3):468-510, 2011.

G. Menzio, S. Shi, and H. Sun. A monetary theory with non-degenerate distributions. Journal of Economic Theory, 148(6):2266-2312, 2013.

P. Milgrom and I. Segal. Envelope theorems for arbitrary choice sets. Econometrica: Journal of the Econometric Society, 70(2):583-601, 2002.

P. Milgrom and C. Shannon. Monotone comparative statics. Econometrica: Journal of the Econometric Society, 62(1):157-180, 1994.

H. Miyazaki. The rat race and internal labor markets. The Bell Journal of Economics, 8(2):394-418, 1977.

E. R. Moen. Competitive search equilibrium. Journal of Political Economy, 105(2):385-411, 1997. 
E. R. Moen and Å. Rosén. Incentives in competitive search equilibrium. The Review of Economic Studies, 78(2):733-761, 2011.

D. T. Mortensen and R. Wright. Competitive pricing and efficiency in search equilibrium. International Economic Review, 43(1):1-20, 2002.

R. B. Myerson. Mechanism design by an informed principal. Econometrica: Journal of the Econometric Society, 51(6):1767-1797, 1983.

M. Peters. Ex ante price offers in matching games non-steady states. Econometrica: Journal of the Econometric Society, 59(5):1425-1454, 1991.

C. A. Pissarides. Equilibrium unemployment theory. Cambridge, MA: MIT press, 2000.

J. Riley. Informational equilibrium. Econometrica: Journal of the Econometric Society, 47(2):331-359, 1979.

M. Rothschild and J. Stiglitz. Equilibrium in competitive insurance markets: An essay on the economics of imperfect information. The Quarterly Journal of Economics, 90(4):629-649, 1976.

S. Severinov. An efficient solution to the informed principal problem. Journal of Economic Theory, 141(1): 114-133, 2008.

S. Shi. Frictional assignment. I. Efficiency. Journal of Economic Theory, 98(2):232-260, 2001.

S. Shi. A directed search model of inequality with heterogeneous skills and skill-biased technology. The Review of Economic Studies, 69(2):467-491, 2002.

S. Shi. Directed search for equilibrium wage-tenure contracts. Econometrica: Journal of the Econometric Society, 77(2):561-584, 2009.

R. Shimer. The assignment of workers to jobs in an economy with coordination frictions. Journal of Political Economy, 113(5):996-1025, 2005.

M. Spence. Product differentiation and performance in insurance markets. Journal of Public Economics, 10 (3):427-447, 1978.

C. Wilson. A model of insurance markets with incomplete information. Journal of Economic Theory, 16(2): 167-207, 1977. 